\title{
Validation of Wichner Predictive Model for Chronic Oxidation by Moisture of Nuclear Graphite
}

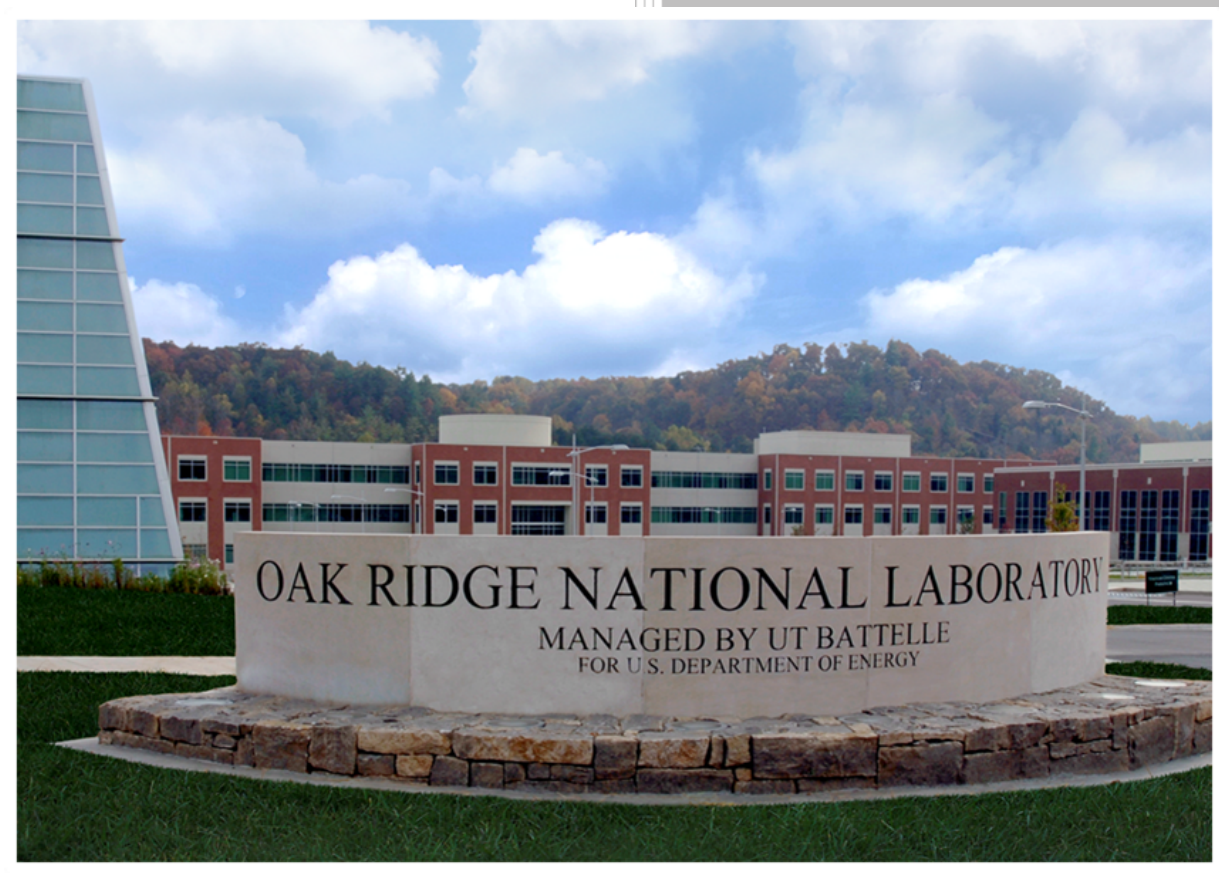

\section{Approved for public release. Distribution is unlimited.}




\section{DOCUMENT AVAILABILITY}

Reports produced after January 1, 1996, are generally available free via US Department of Energy (DOE) SciTech Connect.

Website www.osti.gov

Reports produced before January 1, 1996, may be purchased by members of the public from the following source:

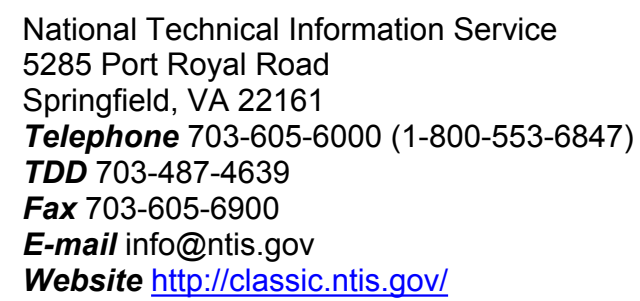

Reports are available to DOE employees, DOE contractors, Energy Technology Data Exchange representatives, and International Nuclear Information System representatives from the following source:

Office of Scientific and Technical Information

PO Box 62

Oak Ridge, TN 37831

Telephone 865-576-8401

Fax 865-576-5728

E-mail reports@osti.gov

Website http://www.osti.gov/contact.html

This report was prepared as an account of work sponsored by an agency of the United States Government. Neither the United States Government nor any agency thereof, nor any of their employees, makes any warranty, express or implied, or assumes any legal liability or responsibility for the accuracy, completeness, or usefulness of any information, apparatus, product, or process disclosed, or represents that its use would not infringe privately owned rights. Reference herein to any specific commercial product, process, or service by trade name, trademark, manufacturer, or otherwise, does not necessarily constitute or imply its endorsement, recommendation, or favoring by the United States Government or any agency thereof. The views and opinions of authors expressed herein do not necessarily state or reflect those of the United States Government or any agency thereof. 
Materials Science and Technology Division

\title{
VALIDATION OF WICHNER PREDICTIVE MODEL FOR CHRONIC OXIDATION BY MOISTURE OF NUCLEAR GRAPHITE
}

\author{
Cristian Contescu
}

September 2019

Prepared by OAK RIDGE NATIONAL LABORATORY

Oak Ridge, TN 37831-6283

managed by

UT-BATTELLE, LLC

for the

US DEPARTMENT OF ENERGY

under contract DE-AC05-00OR22725 



\section{CONTENTS}

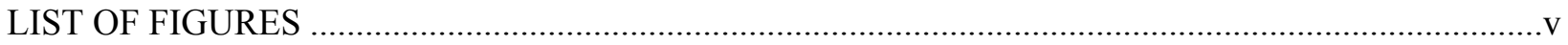

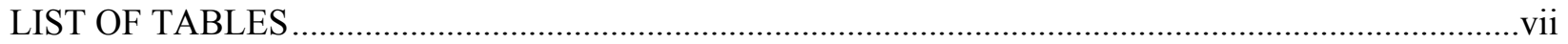

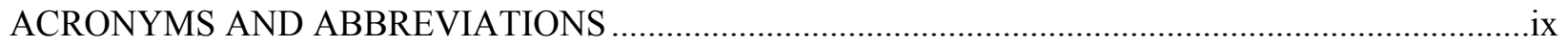

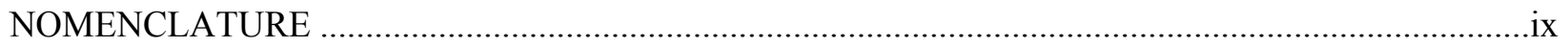

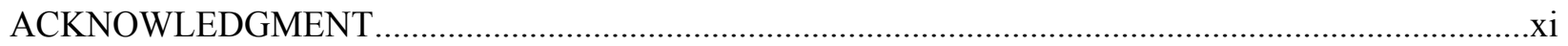

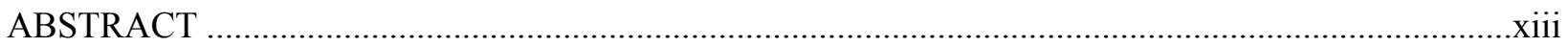

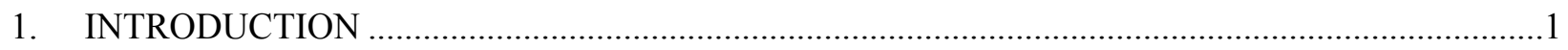

2. OVERVIEW OF RESEARCH STRATEGY BASED ON WICHNER MODEL ..............................

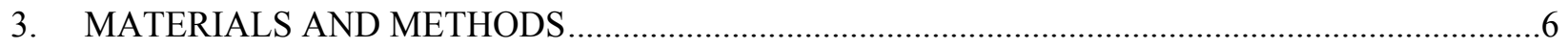

3.1 PROPERTIES OF FOUR NUCLEAR GRAPHITE GRADES .........................................

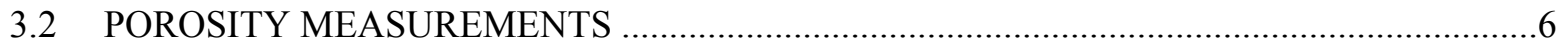

3.3 OUTLINE OF EXPERIMENTAL PROCEDURES AND VALIDATION STEPS ..................

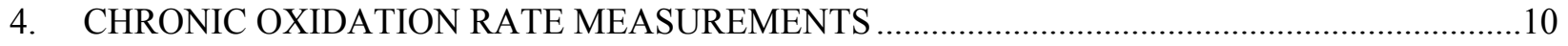

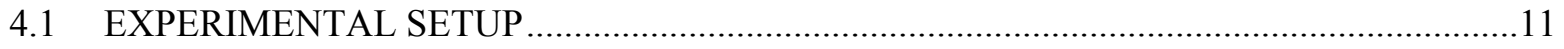

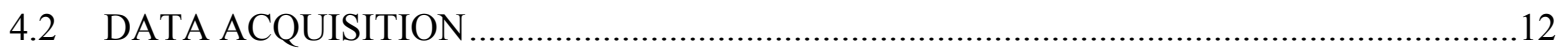

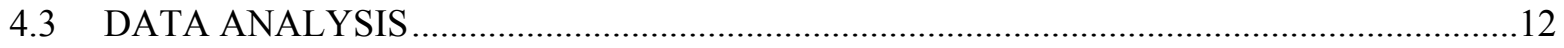

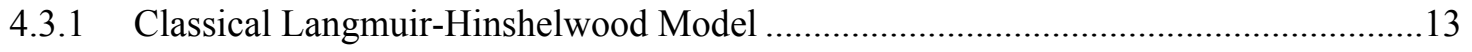

4.3.2 Boltzmann-enhanced Langmuir-Hinshelwood Model .................................................15

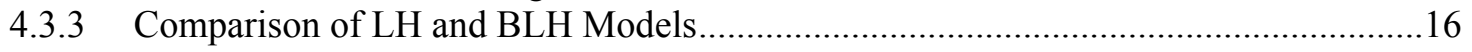

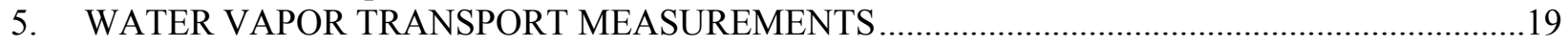

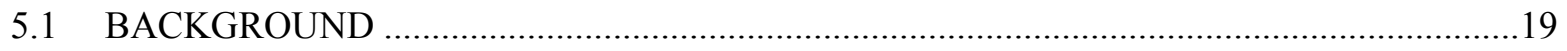

5.1.1 Gas Permeation through Porous Media ...............................................................19

5.1.2 Gas Diffusion through Porous Media ...................................................................19

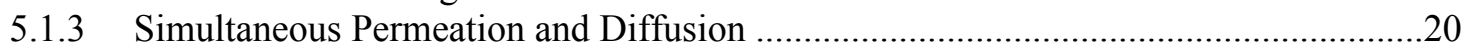

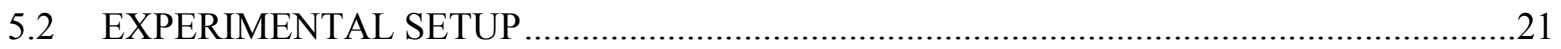

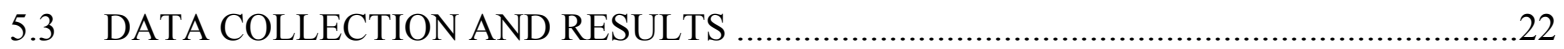

6. MODELING OXIDIZED LAYER DEVELOPMENT IN TRANSIENT CONDITIONS .................28

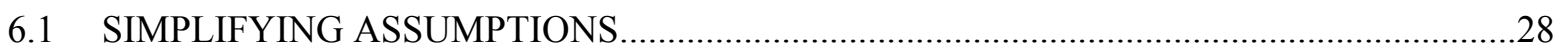

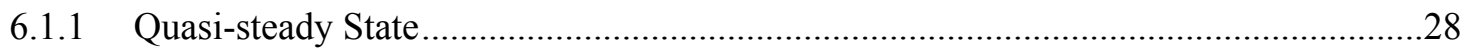

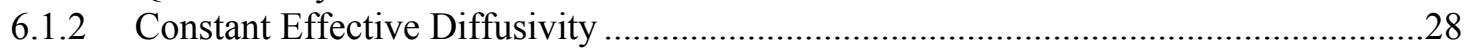

6.1.3 Linearized Oxidation Kinetics derived from the LH Model......................................28

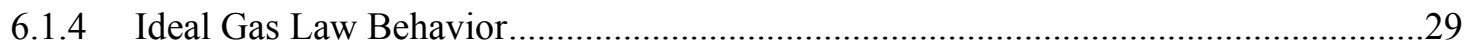

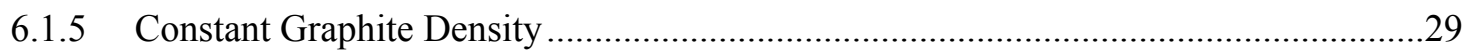

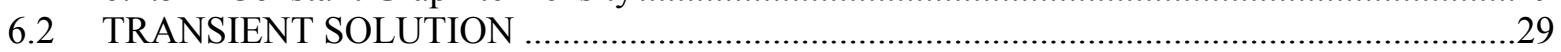

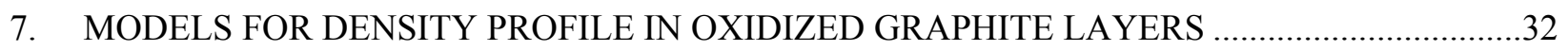

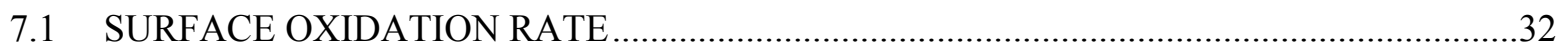

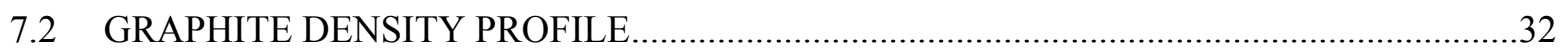

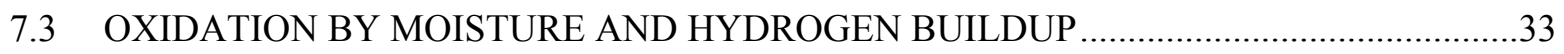

7.3.1 Coupled Transport Equations for Water and Hydrogen ...........................................33

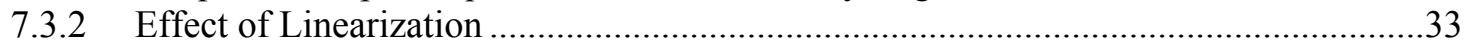

7.3.3 Estimation of Water and Hydrogen Concentration Profiles in a Finite Slab ................34

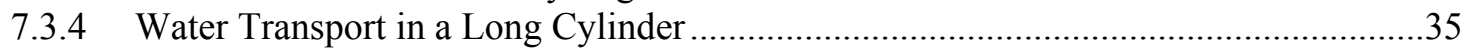

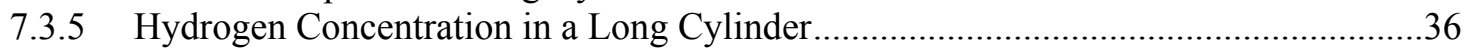

7.3.6 Surface Oxidation Rate by Moisture of a Long Cylinder .............................................37

7.3.7 Efficiency of Oxidation by Moisture of a Long Graphite Cylinder...............................37

8. MEASUREMENTS ON DENSITY PROFILES OF OXIDIZED GRAPHITE SPECIMENS............39

8.1 NEW OXIDATION EXPERIMENTS IN CONTROLLED CONDITIONS ..........................39

8.1.1 Preparation of Specimens Oxidized at Higher Weight Loss.........................................39 
8.2 EXPERIMENTAL OBSERVATION OF OXIDIZED LAYERS …....................................41

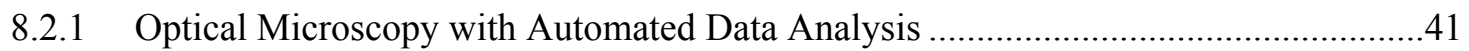

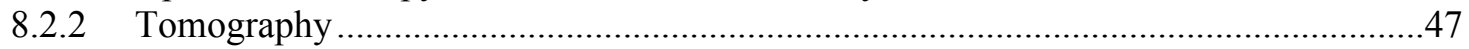

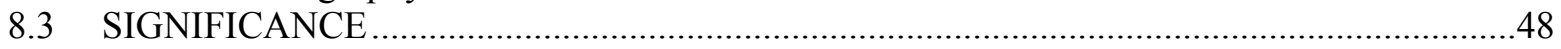

9. COMPARISON BETWEEN PREDICTED AND OBSERVED OXIDATION PROFILES...............50

9.1 ESTIMATION OF WATER AND HYDROGEN CONCENTRATION PROFILES ..............50

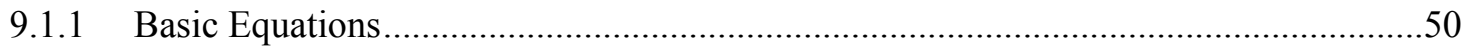

9.1.2 Linearized LH Model versus Complete Version of LH and BLH Models ...................50

9.1.3 Calculation Sequence for Linearized LH Model and Full LH and BLH Models .........51

9.2 RESULTS

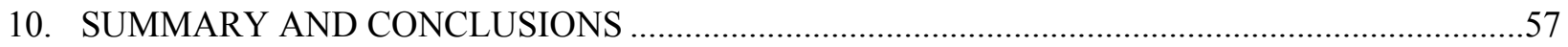

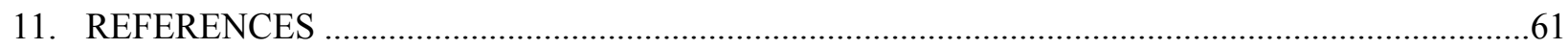

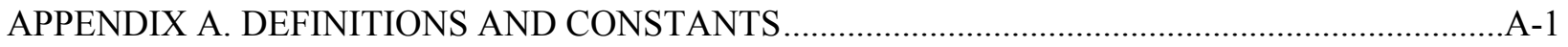

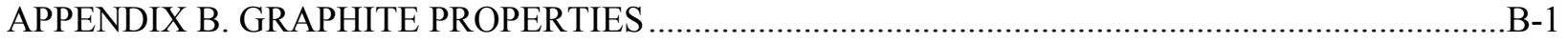

APPENDIX C. WATER PENETRATION AND HYDROGEN BUILDUP PROFILES IN

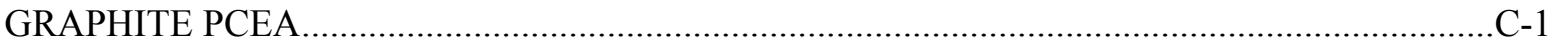

APPENDIX D. WATER PENETRATION AND HYDROGEN BUILDUP PROFILES IN

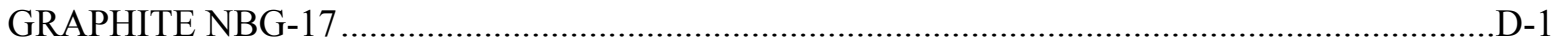

APPENDIX E. WATER PENETRATION AND HYDROGEN BUILDUP PROFILES IN

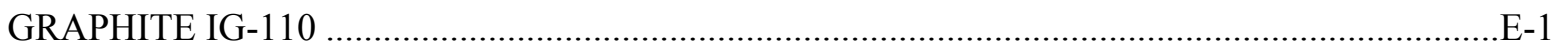

APPENDIX F. WATER PENETRATION AND HYDROGEN BUILDUP PROFILES IN

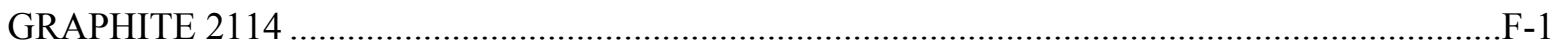




\section{LIST OF FIGURES}

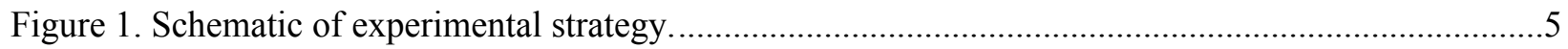

Figure 2. Optical microscopy images in polarized light of the four graphite grades studied. ....................6

Figure 3. Pore size distribution from mercury intrusion porosimetry .................................................

Figure 4. Pore size distribution from nitrogen adsorption measurements. ...............................................

Figure 5. Schematic of experimental setup for chronic graphite oxidation by moisture...........................11

Figure 6. Experimental oxidation rate data (symbols) and their fitting by the LH kinetic model

(continuous lines) at various temperatures.

Figure 7. Experimental oxidation rate data (symbols) and their fitting by the new BLH kinetic

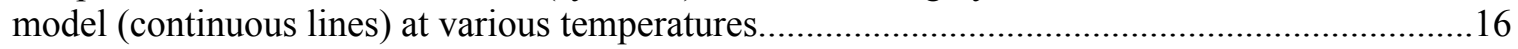

Figure 8. Comparison of fitting quality by using the LH model with parameters from Table 4...............17

Figure 9. Comparison of fitting quality by using the BLH model with parameters from Table 5.............18

Figure 10. Schematic of DMPC instrument used for measurements of water vapor transmission

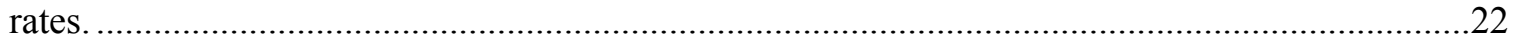

Figure 11. Schematic of test sequences in DMPC: (a) and (c) combined diffusion and permeation of water vapor and helium permeation; (b) water diffusion only, no He flow. Adapted from Evans et al. $1961^{2}$.

Figure 12. Experimental results showing water transmission rates as a function of pressure difference across the graphite specimens.

Figure 13. Comparing graphite structural factors measured from diffusion experiments at ORNL and INL versus graphite density and total porosity fraction.

Figure 14. Effect of temperature on the time required for stabilization of a fully developed density profile in the oxidized layer.

Figure 15. Comparison of oxidation rate variation with $\mathrm{P}_{\mathrm{H} 2 \mathrm{O}}$ obtained from fitting with experimental results data for all graphite grades at 800,900 and $1000^{\circ} \mathrm{C}$.

Figure 16. Examples of pixel intensity distributions extracted from images of pristine and oxidized NBG-17 specimens.

Figure 17. Color-coded visualization of fine pores in the oxidized layer after oxidation by water of PCEA graphite (6 mm diameter).

Figure 18. Color-coded visualization of fine pores in the oxidized layer after oxidation by water of NBG-17 graphite (4 mm diameter).

Figure 19. Color-coded visualization of fine pores in the oxidized layer after oxidation by water of IG-110 graphite (4 $\mathrm{mm}$ diameter).

Figure 20. Color-coded visualization of fine pores in the oxidized layer after oxidation by water of 2114 graphite ( $5 \mathrm{~mm}$ diameter).

Figure 21. Density profiles of oxidized layers fitted with exponential decay function.

Figure 22. Comparison between tomography and microscopy results for oxidized PCEA specimens ( $6 \mathrm{~mm}$ diameter).

Figure 23. Comparison of fitted density profiles measured by microscopy (left panel) and tomography (right panel) for two halves of the same PCEA specimens oxidized at four temperatures.

Figure 24. Comparison among predictions of various models for penetration depth at $50 \%$ oxidant concentration drop and experimental results at 50\% graphite density drop for specimens oxidized at various temperatures

Figure 25. Polarized light microscopy images of pristine and oxidized graphite IG-110, showing lack of surface-localized oxidized layer......

Figure 26. Updated schematic of experimental strategy showing correlations between various groups of results 



\section{LIST OF TABLES}

Table 1. Properties of the four graphite grades used in this study.

Table 2. Chemical impurities in helium coolant and design temperatures in several HTGR projects.

Table 3. Graphite specimen properties and range of experimental conditions for each grade

Table 4. Langmuir-Hinshelwood analysis results for four graphite grades, showing temperature dependence of kinetic constants of Eq. (11).

Table 5. Boltzmann-modified Langmuir-Hinshelwood analysis results of four graphite grades, showing temperature dependence of kinetic constants in Eq. (12)

Table 6. Fitting quality parameters for LH and BLH models compared between graphite grades.

Table 7. Experimental details for water transport measurements and effective diffusivity results

Table 8. Structural and kinetic parameters used for estimation of time needed for development of a stable density profile.

Table 9. Specimens oxidized at higher weight loss for observation of oxidized layer.

Table 10. Fitted parameters of exponential decay functions associated with oxidized layer profiles. 



\section{ACRONYMS AND ABBREVIATIONS}

$\begin{array}{ll}\text { ADM } & \text { advective-diffusive model } \\ \text { ART } & \text { Advanced Reactor Technologies } \\ \text { BLH } & \text { Boltzmann-modified Langmuir-Hinshelwood } \\ \text { DGM } & \text { dusty gas model } \\ \text { DMPC } & \text { dynamic moisture permeation cell } \\ \text { DOE } & \text { US Department of Energy } \\ \text { HTGR } & \text { high-temperature gas-cooled reactor } \\ \text { INL } & \text { Idaho Nuclear Laboratory } \\ \text { LH } & \text { Langmuir-Hinshelwood } \\ \text { NRC } & \text { Nuclear Regulatory Commission } \\ \text { ORNL } & \text { Oak Ridge National Laboratory } \\ \text { PIRT } & \text { Phenomena Identification and Ranking Table } \\ \text { TAG } & \text { thermogravimetric analyzer (French: thermo-analyseur gravimetrique) } \\ \text { VHTR } & \text { very-high-temperature gas-cooled reactor }\end{array}$

\section{NOMENCLATURE}

$A=$ cross section of transport area in the porous medium, $\mathrm{m}^{2}$

$a \quad=$ radius of cylindrical graphite specimens

$\Delta C=$ logarithmic mean water vapor concentration difference between two streams, $\mathrm{kg} \cdot \mathrm{m}^{3}$

$C_{o x} \quad=$ concentration of oxidant, $\mathrm{mol} / \mathrm{m}^{3}$ voids $=\mathrm{mol} \cdot \mathrm{m}^{-3}$

$C_{\text {sat }}=$ concentration of saturated water vapor at test temperature, $\mathrm{kg} \cdot \mathrm{m}^{-3}$

$d_{\text {hyd }} \quad=$ hydraulic diameter of oxidation rates test tube, $\mathrm{m}$

$D \quad=$ van der Waals molecular diameters, $\mathrm{nm}$

$D_{A B} \quad=$ binary diffusion coefficient in gas phase of $\mathrm{AB}$ gas mixture, $\mathrm{m}^{2} \cdot \mathrm{s}^{-1}$

$D_{e f f} \quad=$ effective diffusion coefficient in porous medium, $\left(\mathrm{m}^{3}{ }_{\text {voids }}\right) \mathrm{m}^{-1} \mathrm{~s}^{-1}=\mathrm{m}^{2} \cdot \mathrm{s}^{-1}$

$D_{\text {global }}=$ global diffusion coefficient in presence of regular and Knudsen diffusion, $\mathrm{m}^{2} \cdot \mathrm{s}^{-1}$

$E_{\mathrm{i}} \quad=$ activation energy of oxidation reactions, $\mathrm{J} \cdot \mathrm{mol}^{-1}$

$F_{\text {diff }}=$ diffusion mass flux, $\mathrm{kg} \cdot \mathrm{m}^{-2} \cdot \mathrm{s}^{-1}$

$F_{\text {perm }}=$ permeation mass flux, $\mathrm{kg} \cdot \mathrm{m}^{-2} \cdot \mathrm{s}^{-1}$

$k_{i} \quad=$ pre-exponent factor in Arrhenius equation, various units $\left(\mathrm{s}^{-1} \mathrm{~Pa}^{-1}\right.$ or s $\left.^{-1}\right)$

$K_{i} \quad=$ rate constant of individual steps in a complex mechanistic model in chemical kinetics, various units

$h \quad=$ mass transfer coefficient

$j=$ mass permeability coefficient, $\mathrm{kg} \cdot \mathrm{m}^{-1} \cdot \mathrm{s}^{-1} \cdot \mathrm{Pa}^{-1}$

$J \quad=$ gas permeance, $\mathrm{kg} \cdot \mathrm{m}^{-2} \cdot \mathrm{s}^{-1} \cdot \mathrm{Pa}^{-1}$

$L \quad=$ thickness of porous medium, $\mathrm{m}$

$\mathrm{m}(\mathrm{T})=$ temperature-dependent kinetic order for water in BLH equation, no units

$\dot{m} \quad=$ mass flow rate, $\mathrm{kg} \mathrm{s}^{-1}$

$\dot{M}_{i / o} \quad=$ molar flow rate (incoming / outgoing), mole. $\mathrm{s}^{-1}$

$M_{w} \quad=$ molar mass of water, 18 mol.g ${ }^{-1}$

$n \quad=$ kinetic order for hydrogen in LH and BLH equations $(n=0.5)$

$N=$ stoichiometric coefficient, $\left(\mathrm{mol}_{\text {oxidant }}\right)\left(\mathrm{mol}_{\text {carbon_oxidized }}\right)^{-1}$ no units

$P_{\text {sat }}=$ saturated water vapor pressure in gas at test temperature, $\mathrm{Pa}$

$P_{u} \quad=$ partial pressure of water vapor in unsaturated gas, $\mathrm{Pa}$ 


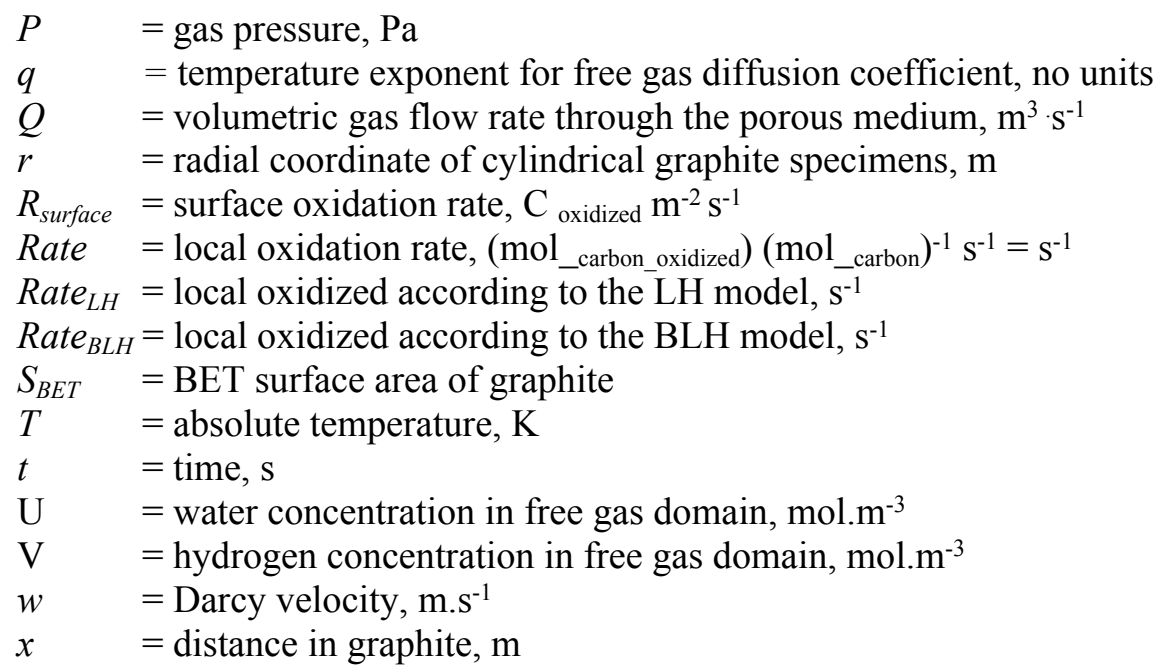

\section{Subscripts}

$\begin{array}{ll}\mathrm{u} & \text { water } \\ \mathrm{v} & \text { hydrogen } \\ \text { LH } & \text { Langmuir-Hinshelwood } \\ \text { BLH } & \text { Boltzmann-modified Langmuir-Hinshelwood }\end{array}$

\section{Greek symbols}

$\alpha \quad=$ factor showing the drop of water concentration or graphite density concentration at any position inside graphite

$\beta=$ porous media structure factor, scaling down free gas diffusivity to effective diffusivity in pores, no units

$\varepsilon \quad=$ local fraction of transport pores, $\left(\mathrm{m}^{3}{ }_{-}\right.$voids $)\left(\mathrm{m}^{3} \text { carbon }\right)^{-1}$; no units

$\eta \quad=$ oxidation efficiency (equivalent to Thiele modulus)

$\kappa \quad=$ gas-phase permeability, $\mathrm{m}^{2}$

$\lambda \quad=$ mean free path of gas molecules

$\mu \quad=$ gas viscosity, $\mathrm{Pa} \cdot \mathrm{s}$

$\rho_{\text {gas }} \quad=$ gas density, $\mathrm{kg} \cdot \mathrm{m}^{-3}$

$\rho=\quad=$ local molar density of graphite, $\mathrm{mol} \_$carbon $/ \mathrm{m}^{3}=\mathrm{mol} . \mathrm{m}^{-3}$

$\theta \quad=$ scaling parameter in the expression of $m(T), \mathrm{K}$

$v \quad=$ gas velocity in pores, $\mathrm{m} \cdot \mathrm{s}^{-1}$

$\tau=$ characteristic dimension for concentration or density decay in the oxidized layer, $\mu \mathrm{m}$ 


\section{ACKNOWLEDGMENT}

This multi-year project was funded by the Nuclear Energy Office of the US Department of Energy through the New Generation Nuclear Plant and Advanced Reactor Technologies programs and was conducted at Oak Ridge National Laboratory (ORNL) as a collaborator for Idaho Nuclear Laboratory (INL).

Throughout the work, the author received help and advice from many colleagues, of which the first to list here is Dr. Robert Wichner (retired). He was invaluable in sharing his past experience with modeling graphite corrosion by oxidation, and he designed the strategy and then did the groundwork for developing algorithms for linking together various parts of the experimental program. A significant contribution came from Dr. Robert Mee (University of Tennessee, Knoxville) who managed the complicated parameter fitting of hundreds of experimental data points to pre-designed model equations. Encouragement and support came also from Dr. Tim Burchell (ORNL) and Dr. William Windes (INL), and valid criticisms and suggestions came from my colleagues at ORNL (Dr. Nidia Gallego and Dr. David Arregui-Mena) and INL (Dr. Joshua Kane). The daily work in the lab was supported by post-doctoral associates (Dr. Yoonjo (Jo Jo) Lee, ORNL) and post-masters associates (Mr. Peng Wang, Tsinghua University). The skills of our talented technical associates, Ashli Clark, Tony McBee, Ed Reed, and Denny Wilson ensured the continuous operation of equipment, computer programs and sample preparation and were an invaluable resource that contributed to the timely completion of this work. 



\begin{abstract}
This document reports the results of multi-year extensive research on chronic oxidation of nuclear graphite by traces of moisture that might be present in the helium coolant of high-temperature gas-cooled reactors (HTGRs). The research focuses on four grades of nuclear graphite that are considered potential candidates for component fabrication in advanced nuclear reactors. The four grades are (1) PCEA (medium-fine grain, extruded, manufactured by GrafTech Inc., USA); (2) NBG-17 (medium-fine grain, vibrationally molded, manufactured by SGL Carbon, Germany/France); (3) IG-110 (microfine grain, isostatically molded, manufactured by Toyo Tanso, Japan); and (4) 2114 (microfine grain, isostatically pressed, manufactured by Mersen, USA). The experimental part of this research comprised three objectives that were consistently pursued for each graphite material: (1) accelerated oxidation tests in well-controlled conditions (temperature, gas composition) and analysis of chemical kinetic models and of their parameters for each graphite grade; (2) measurements of water vapor transport rates from $\mathrm{H}_{2} \mathrm{O} / \mathrm{He}$ mixtures through thin graphite slabs for estimation of the water effective diffusivity specific to each material; (3) controlled oxidation of graphite specimens in $\mathrm{H}_{2} \mathrm{O} / \mathrm{He}$ mixtures and characterization of the effects of oxidation temperatures on the density profiles of oxidized layers. These three groups of experimental results were interpreted using the model developed by Wichner et al. for predicting the penetration depth of oxidants (moisture or oxygen) as a function of oxidation conditions. Basically, starting from the kinetic and transport properties of each graphite grade, the ultimate goal was predicting the stable density profile in the oxidized layer and its dependence on the oxidation temperature. The profiles obtained this way were then compared against direct observations of oxidized layers in specimens obtained in well-controlled oxidation conditions. The general agreement between predictions and observations validated the general applicability of the Wichner model.

The results obtained in this project were presented at annual Department of Energy-Advanced Reactor Technologies program reviews and at specialist meetings and conferences and were published in Oak Ridge National Laboratory (ORNL) reports and in professional journals. The results published in 2018 had an immediate impact in 2019, when a group from three US universities used ORNL kinetic data as input information in a complex simulation of chronic oxidation by moisture using the same graphite grades during long-time operation in a modular high-temperature gas-cooled reactor [Wang et al. Ann. Nucl. Energy 131 (2019) 483-495]. Their conclusion, in line with the conclusions obtained at the end of this project, was that chronic graphite oxidation by moisture during normal operation conditions of an HTGR will be limited to a thin surface layer of graphite blocks at the reactor bottom, where the highest temperatures are. That will not affect the integrity of graphite components and reactor safety, provided the impurity concentration in the helium coolant is rigorously controlled and maintained at the design specifications.
\end{abstract}





\section{INTRODUCTION}

Helium (He) coolant in high-temperature gas-cooled reactors (HTGRs) and very-high-temperature gascooled reactors (VHTR) will be strictly controlled for chemical composition to eliminate unwanted impurities that may affect the operation and the physical integrity of graphite core components. However, the coolant will have a slightly oxidizing potential, which is needed to protect metallic components against carburization. The main oxidizing impurity will be water at extremely low concentrations, less than $1 \mathrm{ppm}$ vol. at a total gas pressure of several MPa. Moisture is the gas impurity that is the most difficult to control. Even at this very low concentration, water may react with the graphite components (moderator, fuel elements, reflector, core support posts) at the high temperatures of reactor operation. Even though oxidation of graphite by water vapor is about 300,000 times slower than oxidation by oxygen at $800{ }^{\circ} \mathrm{C}$ and $0.1 \mathrm{~atm},{ }^{1}$ it is estimated that the surfaces of graphite components will be corroded, extremely slowly but continuously, over the lifetime of the reactor. ${ }^{2}$ To evaluate the long-term effect of this chronic oxidation on the graphite integrity, it is necessary to quantify both the oxidation rates (chemical kinetics) and the oxidant transport (diffusivity) in the graphite material.

In 2007 the US Nuclear Regulatory Commission (NRC) conducted a Phenomena Identification and Ranking Table (PIRT) exercise to identify phenomena that could potentially lead to accidents accompanied by releases of radionuclides. The graphite PIRT panel ${ }^{3}$ identified chronic chemical attack by impurities in the He coolant during normal operation as a medium-importance, medium-knowledge phenomenon that may affect mechanical integrity and increase the rate of dust formation. The same panel identified strength changes induced by oxidation, temperature, and irradiation as a high-importance, medium-knowledge phenomenon. The PIRT panel emphasized the need to understand the effect of surface oxidation of core graphite and to elaborate analytical and predictive models for estimating the extent of strength loss caused by graphite oxidation in relation to the weight loss and oxidation conditions.

In 2009 NRC funded Oak Ridge National Laboratory (ORNL) to conduct a panel workshop to review the PIRT findings, to compare them against current Department of Energy (DOE) plans, and to identify potential technology gaps. The panel ${ }^{4}$ emphasized that new specific information is needed to provide the technical base for evaluation of HTGR design readiness for certification and licensing. In particular, the workshop panel highlighted the need to develop capabilities for comprehensive modeling of graphite oxidation, based on accurate knowledge of both oxidation kinetics and transport of oxidant species within graphite structures. It was emphasized that specific rate data were needed for oxidation at low concentrations of $\mathrm{H}_{2} \mathrm{O}$ and $\mathrm{H}_{2}$ in $\mathrm{He}$ to evaluate the long-term behavior of graphite components under normal operating conditions. These recommendations were the starting point of a multi-year research project at ORNL dedicated to characterization of kinetics and transport processes involved in chronic oxidation of nuclear graphite by moisture. The goal was to develop predictive models for analysis of graphite component behavior during long-term exposure to the oxidizing impurities that may be present in the He coolant of HTGRs during normal operation.

A first step in this direction was the theoretical analysis of the problem. In 2008-2009, Dr. Robert Wichner, a former ORNL scientist, then retired, assumed the task of reviewing the status of graphite oxidation models, focusing on achieving a sufficient basic understanding of the phenomena to enable the proper planning and development of a coherent experimental project that would eventually provide useful information for computer code development. The review of perhaps 50 years of graphite oxidation research published as an ORNL report ${ }^{5}$ underscored the intrinsic relationship between kinetics and transport phenomena for understanding possible strength effects on graphite components. Toward this goal, general equations were derived for oxidized layer profiles in graphite specimens of various shapes, with a few simplifying assumptions. A further open literature publication ${ }^{6}$ provided examples of coupled 
kinetic and transport equations solved by spreadsheet calculations which, although approximate, agreed well with the few experimental results available at the time for oxidized layer profiles of graphite oxidized by air. ${ }^{7}$ The oxidation analysis by Wichner et al. emphasized the impact of the effective diffusivity of the oxidant in graphite, which controls the profile and penetration depth of oxidation. That motivated our direct measurements of water diffusivity for each grade of graphite analyzed in this project. A second conclusion was that the maximum penetration depth of the oxidant depends on temperature, not on the time of exposure or the level of weight loss. That conclusion, which was first verified for oxidation of graphite by air $^{8,9}$ and by steam ${ }^{10}$, was further used to select conditions for oxidation by moisture for model validation experiments.

The ORNL experimental program had three main goals during 2012-2019: (1) collection of a statistically significant number of oxidation rate data and development of kinetic models describing graphite oxidation in $\mathrm{He}$ with low concentrations of $\mathrm{H}_{2} \mathrm{O}$ and $\mathrm{H}_{2} ;(2)$ measurement of water transport through graphite slabs in $\mathrm{H}_{2} \mathrm{O}+\mathrm{He}$ mixtures; and (3) analysis and quantification of oxidation layer profiles in graphite specimens oxidized by moisture at various temperatures. The highest oxidation weight loss in the kinetic experiments of pursued under goal (1) described above was less than $1 \mathrm{wt} \%$; the weight loss of oxidized specimens obtained under goal (3) above was 5-6 wt \% in order to facilitate the observation of oxidation layers. All experiments were performed with the following four grades of graphite considered as possible candidates for use in HTGR reactors:

- $\quad$ PCEA (medium-fine grains, extruded, manufactured by GrafTech Inc., USA)

- NBG-17 (medium-fine grains, vibrationally molded, manufactured by SGL Carbon, Germany/France)

- IG-110 (superfine grains, isostatically molded, manufactured by Toyo Tanso, Japan)

- 2114 (superfine grains, isostatically pressed, manufactured by Mersen, USA)

Partial results obtained during the progress of the project were presented at the annual DOE-Advanced Reactor Technologies (ART) program reviews ${ }^{11,12,13,14,15}$ and professional conferences ${ }^{16,17,18,19,20,21}$ and were published either as ORNL reports $22,23,24,25,26,27,28$ or in open-access journals. ${ }^{29}$

Soon after publication in 2018, the paper ${ }^{29}$ on chronic oxidation kinetics of the four graphite grades had its first impact. A group of researchers from University of Michigan, University of Idaho, and Ohio State University used ORNL published data as the input kinetic information for a complex simulation of chronic oxidation by moisture in the He coolant of graphite fuel elements during long-term operation in a modular HTGR. ${ }^{30}$ Selecting the most probable coolant gas composition during normal operation of an HTGR, they modeled the fluid flow, heat and mass transfer, chemical reactions, and material degradation in prototype $3 \mathrm{D}$ graphite structures. This type of multiphysics simulation was beyond the scope of the present project, as our main goal was collecting experimental data and interpreting them using the simplified model by Wichner et al. However, one cannot fail to notice the utility of this ORNL project and the immediate attention it received from other specialists.

The report summarizes the background, methodology, and experimental results and conclusions of the project. To provide theoretical support for data interpretation and conclusion, brief excerpts from Wichner's original works are also included. However, this is not an exhaustive presentation of the work performed during the multi-year project on chronic oxidation. References to annual ORNL reports and publications are frequently made to keep this final report within an acceptable length. 


\section{OVERVIEW OF RESEARCH STRATEGY BASED ON WICHNER MODEL}

In 2008, Dr. Robert Wichner took upon himself the task of reviewing more than 50 years of literature publications on the problem of graphite oxidation in relation to the use of graphite as a structural or moderator component in gas-cooled reactors. In particular, he focused on long-term oxidation caused by gas impurities (such as moisture) in the He coolant during normal operation. His efforts led to a convenient analytical procedure for evaluation of the penetration depth of oxidation and of the density profile in the oxidized layer developed in quasi-steady state oxidation conditions on exposed graphite components. ${ }^{5,6}$ This short overview will introduce the main concepts of the model. More in-depth justifications are provided at a more appropriate place.

The Wichner model is based on several simplifying assumptions, which replace the burden of heavy mathematics with elegant analytical solutions that can be easily handled using spreadsheet applications (e.g., Excel). The development of the transient oxidation profile was derived on the assumptions of linearized equations for chemical oxidation rates and constant graphite density. The oxidant transport in graphite was modeled through molecular diffusion models (Fick's laws), and Knudsen and surface diffusion were neglected. In the absence of accurate measurements of gas diffusivity in various graphite structures, Wichner used the notion of effective diffusivity, defined as a fraction of the free gas diffusivity at the corresponding temperatures and pressures. With those restrictions, the applicability of the original model $^{5,6}$ to fine-grained graphite materials might be limited. Later in the project, we introduced changes to better incorporate the new information obtained from experiments.

The essential steps of Wichner model are presented briefly here. With the above simplifications, the transport equation for oxidant in a semi-infinite graphite slab was expressed as

$$
\frac{\partial u}{\partial t}=\frac{D_{e f f} \partial^{2} u}{\varepsilon \partial x^{2}}-\frac{\text { Rate }_{I} \rho N_{u}}{\varepsilon} .
$$

In this equation, $u$ is the oxidant concentration ( $\mathrm{mol} / \mathrm{m}^{3}$-void), $\varepsilon$ is the local void fraction, $\rho$ is the local density of graphite (mol carbon $\left./ \mathrm{m}^{3}\right), N_{u}$ is a stoichiometric coefficient for the oxidation reaction (mol oxidant used per mol carbon oxidized), and $D_{\text {eff }}$ is the effective oxidant diffusivity in graphite $\left(\mathrm{m}^{2} / \mathrm{s}\right)$ :

$$
D_{e f f}=\beta D_{g a s},
$$

where $\beta$ is a semiempirical parameter which was initially assigned a $\beta=0.01$ value for lack of other information. The rate of chemical oxidation reaction in diffusion-free conditions, (also known as zone I), Rate $_{I}$ was introduced by the linearized equation

$$
\text { Rate }_{L H-l i n}=K_{u} P_{u}=k_{u} \exp \left(-\frac{E_{u}}{R T}\right) P_{u}
$$

where the rate constant $K_{u}$ has the Arrhenius form, which includes the frequency factor $k_{u}$ as preexponent, and temperature $T$ and activation energy $\left(E_{u}\right)$ in the exponential function. $\mathrm{R}$ is the gas constant, and $P_{u}$ is the partial pressure of the oxidant $\left(\mathrm{H}_{2} \mathrm{O}\right)$ in the void space at any given time $(t)$ and position $(x)$. Compared with the widely accepted complete form of the oxidation rate in the Langmuir-Hinshelwood (LH) model $^{1}$, Eq. (4),

$$
\operatorname{Rate}_{(L H)}=\frac{k_{1} \exp \left(-\frac{E_{1}}{R T}\right) P_{H 2 O}}{1+k_{2} \exp \left(-\frac{E_{2}}{R T}\right)\left(P_{H 2}\right)^{n}+k_{3} \exp \left(-\frac{E_{3}}{R T}\right) P_{H 2 O}},
$$


the linearized equation neglects the terms in $P_{\mathrm{H} 2 \mathrm{O}}=P_{u}$ and $P_{\mathrm{H} 2}=P_{v}$ from the denominator but preserves the general trend of $R_{L H \text {-lin }}$ with temperature and $\mathrm{H}_{2} \mathrm{O}$ pressure, $P_{u}$. After replacing $P_{u}=\mathrm{R} T$ based on ideal gas laws, Eq. (1) reduces to

$$
\frac{\partial u}{\partial t}=\frac{D_{e f f} \partial^{2} u}{\varepsilon \partial x^{2}}-\frac{A}{\varepsilon} u
$$

where

$$
A=K_{u} \mathrm{RT} \rho N_{u}=k_{1} \exp \left(-\frac{E_{u}}{\mathrm{RT}}\right) \mathrm{R} T \rho N_{u} .
$$

The differential equation (5) has a simple analytical solution. At large $t$, one can assume that the oxidant concentration has stabilized $(\partial u / \partial t=0)$ and a steady state was reached. In this case, the condensed form of Eq. (5) becomes

$$
0=u^{\prime \prime}-B^{2} u
$$

where

$$
B^{2}=\frac{A}{D_{e f f}} .
$$

The solution of Eq. (7) with boundary conditions $u(x=0)=U$ (the constant oxidant concentration at the exposed surface is equal to that in the free gas volume, $U$ ) and $u(x \rightarrow \infty)=0$ (no oxidation in the bulk at a remote distance from the exposed surface) is

$$
\frac{u(x)}{U}=\exp (-B x) .
$$

This result shows that, at steady-state conditions, the oxidant concentration inside an infinite graphite slab decreases exponentially with the distance from the exposed surface, where the surface concentration is constant $(U)$, and the rate of the exponential decay $(B)$ depends on the ratio between kinetic $(A)$ and transport $\left(D_{\text {eff }}\right)$ factors. The thickness of the oxidized layer can be obtained directly from Eq. (9) using

$$
X_{\alpha}=-\ln (\propto) / B
$$

where $X_{\alpha}$ is the distance below the surface where the oxidant concentration in pores dropped to a certain fraction $\alpha$ from the value at the surface at steady state conditions. The values of $X_{\alpha}$ can be calculated if the kinetic $(A)$ and transport $\left(D_{e f f}\right)$ terms needed for estimation of $B$ are known. The density profile of the oxidized layer is the mirror image of the oxidant concentration profile. On the other hand, the density profile of the oxidized layer can be obtained independently, from physical measurements of specimens oxidized at different conditions (temperatures, oxidant concentrations). It is expected that the distance under the surface where graphite density is a fraction $\alpha$ of its value in the bulk, $x_{\alpha}$, is close to $X_{\alpha}$ calculated from the oxidant distribution profile. Thus, a method to compare and validate the model is at hand, if the kinetic and transport terms are evaluated first.

The above rationale defines the strategy of our experimental work, which is shown schematically in Figure 1. We selected four graphite grades with various properties. They are introduced in Chapter 3 along with details on the experimental methods. Then we measured oxidation rates at various conditions $\left(T, P_{\mathrm{H} 2 \mathrm{O}}, P_{\mathrm{H} 2}\right)$ of the four grades and estimated the kinetic parameters of the oxidation rate equation. Two kinetic models were analyzed, the classical LH model and a new, Boltzmann-modified Langmuir- 
Hinshelwood model (BLH). Details are presented in Chapter 4. This step produced the parameters required for evaluation of $A$ in Eq. (6). Second, we measured water diffusivity from $\mathrm{H}_{2} \mathrm{O} / \mathrm{He}$ mixtures and calculated the effective diffusivity $D_{\text {eff }}$ of the same four graphite grades. This step allowed calculation of $B$ in Eq. (8). Details are available in Chapter 5. With experimental information on kinetics and water transport, using Eq. (9) to estimate steady state oxidation profiles as a function of temperature became possible for each graphite grade. Chapters 6 and 7 explain the steps needed for solving the general reaction and transport equation in porous graphite and discuss the effect of the simplifications listed above. To validate the model, the estimates had to be compared against direct measurements of oxidized layer penetration in specimens oxidized at controlled conditions. For that, in the third experimental step, we produced several specimens of each graphite grade oxidized at constant temperatures and at weight losses high enough for development of a clearly observable oxidized layer. Details are presented in Chapter 8. Finally, Chapter 9 compares model predictions on penetration depth in oxidized graphite against direct observations. Chapter 10 summarizes the work and outlines a few conclusions.

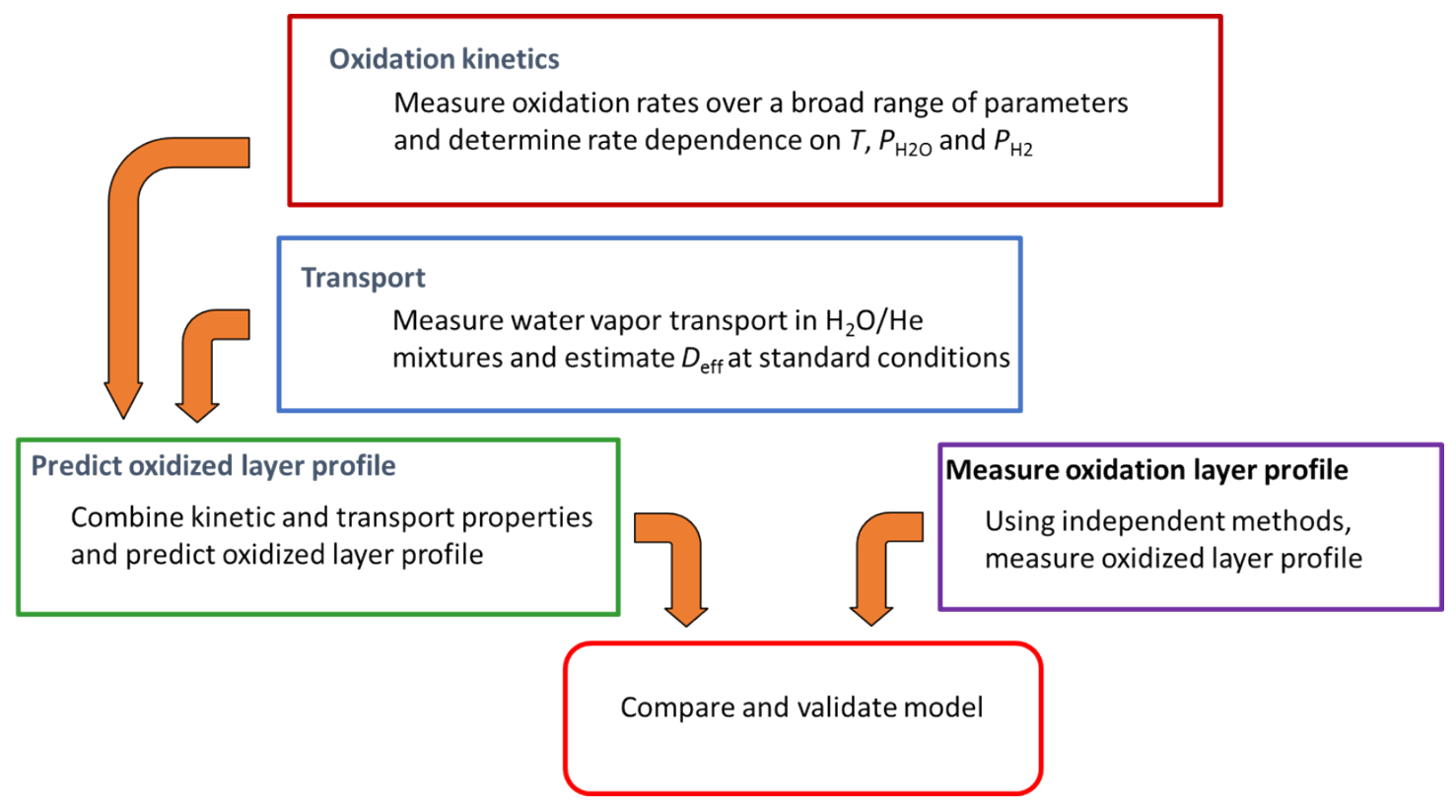

Figure 1. Schematic of experimental strategy. 


\section{MATERIALS AND METHODS}

\subsection{PROPERTIES OF FOUR NUCLEAR GRAPHITE GRADES}

Four grades of nuclear graphite were used in this study (Table 1). Based on grain size, graphite grades PCEA and NBG-17 are classified as medium-fine grades, and the other two (IG-110 and 2114) are superfine grain graphite. ${ }^{1}$ This difference is important, as will be shown below, because the character of water vapor transport by diffusion may be significantly different between medium-fine and superfine grain graphite, and that might influence both the rate of oxidation and the development of the oxidized layer. Figure 2 shows polarized light optical microscopy images of unoxidized graphites. The mosaic images were collected by stitching together 56 individual images collected at $400 \times$ magnification.

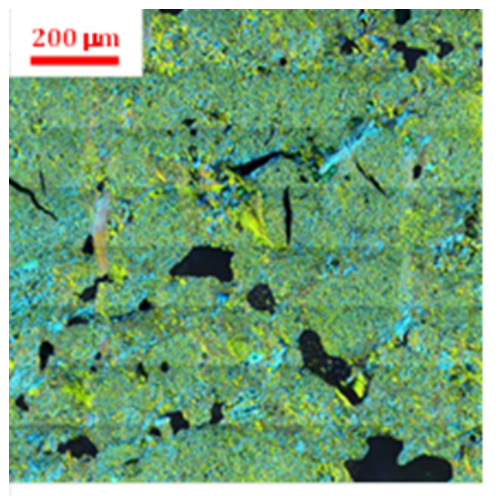

PCEA

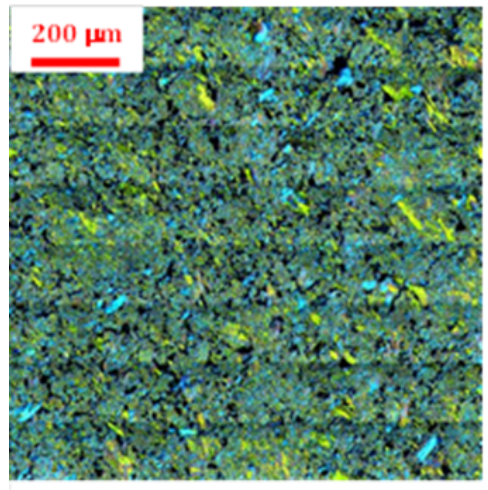

IG-110

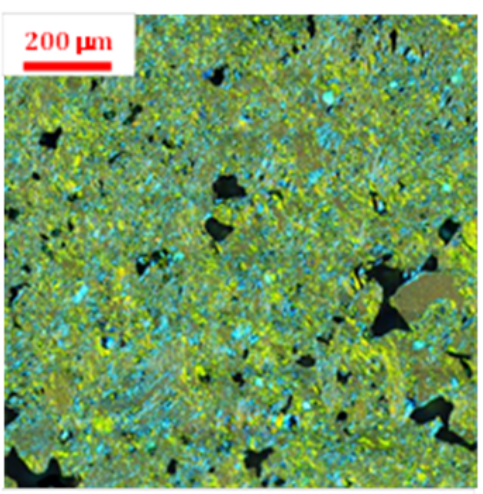

NBG-17

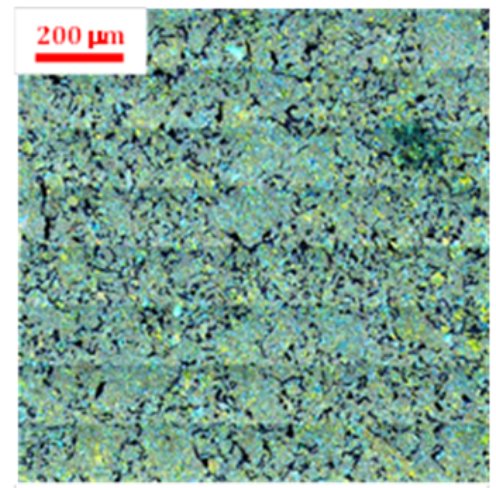

2114

Figure 2. Optical microscopy images in polarized light of the four graphite grades studied.

\subsection{POROSITY MEASUREMENTS}

Nuclear graphite is a porous material. Based on density values, about $17-20 \%$ of its volume is contained in pores, either open or closed (Table 1). Mercury intrusion measurements are the preferred method for characterization of materials with a large variation in pore sizes, from sub-millimeter to nanometer scale (Figure 3). However, in some instances, unwanted damage to graphite may occur at the high pressures needed to force mercury into narrow graphite pores; it would artificially increase the volumes of pores

${ }^{1}$ ASTM D7219-08 (Reapproved 2014), Standard specification for isotropic and near-isotropic nuclear graphites 
smaller than $0.1 \mu \mathrm{m}(100 \mathrm{~nm}) .{ }^{31}$ In those situations, nitrogen adsorption at $77 \mathrm{~K}$ is used as a complementary technique for accessing micropores $(<2 \mathrm{~nm})$ and mesopores $(2-50 \mathrm{~nm})$ in graphite (Figure 4).

Table 1. Properties of the four graphite grades used in this study.

\begin{tabular}{|l|c|c|c|c|c|c|c|c|l|}
\hline \multirow{2}{*}{ Grade } & \multirow{2}{*}{$\begin{array}{c}\text { Classifi- } \\
\text { cation }\end{array}$} & $\begin{array}{c}\text { Grain } \\
\text { size (a) }\end{array}$ & $\begin{array}{c}\text { Apparent } \\
\text { density }\end{array}$ & $\begin{array}{c}\text { Skeleton } \\
\text { density }\end{array}$ & $\begin{array}{l}\text { Porosity } \\
\text { fraction }\end{array}$ & $\begin{array}{c}\text { BET } \\
\text { surface } \\
\text { area (d) }\end{array}$ & $\begin{array}{c}\text { Forming } \\
\text { procedure }\end{array}$ & $\begin{array}{c}\text { Raw } \\
\text { material }\end{array}$ & Vendor \\
\cline { 3 - 8 } PCEA & $\begin{array}{c}\text { medium- } \\
\text { fine }\end{array}$ & 800 & 1.809 & 1.912 & 0.20 & 2.62 & extruded & $\begin{array}{l}\text { petroleum } \\
\text { coke }\end{array}$ & Graftech (USA) \\
\hline $\begin{array}{l}\text { NBG- } \\
17\end{array}$ & $\begin{array}{c}\text { medium- } \\
\text { fine }\end{array}$ & 800 & 1.854 & 2.10 & 0.18 & 1.18 & $\begin{array}{l}\text { vibrationally } \\
\text { molded }\end{array}$ & pitch coke & $\begin{array}{l}\text { SGL } \\
\text { (France/Germany) }\end{array}$ \\
\hline IG-110 & superfine & 20 & 1.779 & 1.937 & 0.22 & 1.38 & $\begin{array}{l}\text { isostatically } \\
\text { pressed }\end{array}$ & $\begin{array}{l}\text { petroleum } \\
\text { coke }\end{array}$ & $\begin{array}{l}\text { Toyo Tanso } \\
\text { (Japan) }\end{array}$ \\
\hline 2114 & superfine & 13 & 1.815 & 2.029 & 0.20 & 2.12 & $\begin{array}{l}\text { isostatically } \\
\text { molded }\end{array}$ & $\begin{array}{l}\text { petroleum } \\
\text { coke }\end{array}$ & Mersen (USA) \\
\hline
\end{tabular}

Notes: (a) vendor supplied information; (b) Archimedes method; (b) helium pycnometry; (d) $\mathrm{N}_{2}$ adsorption at $77 \mathrm{~K}$
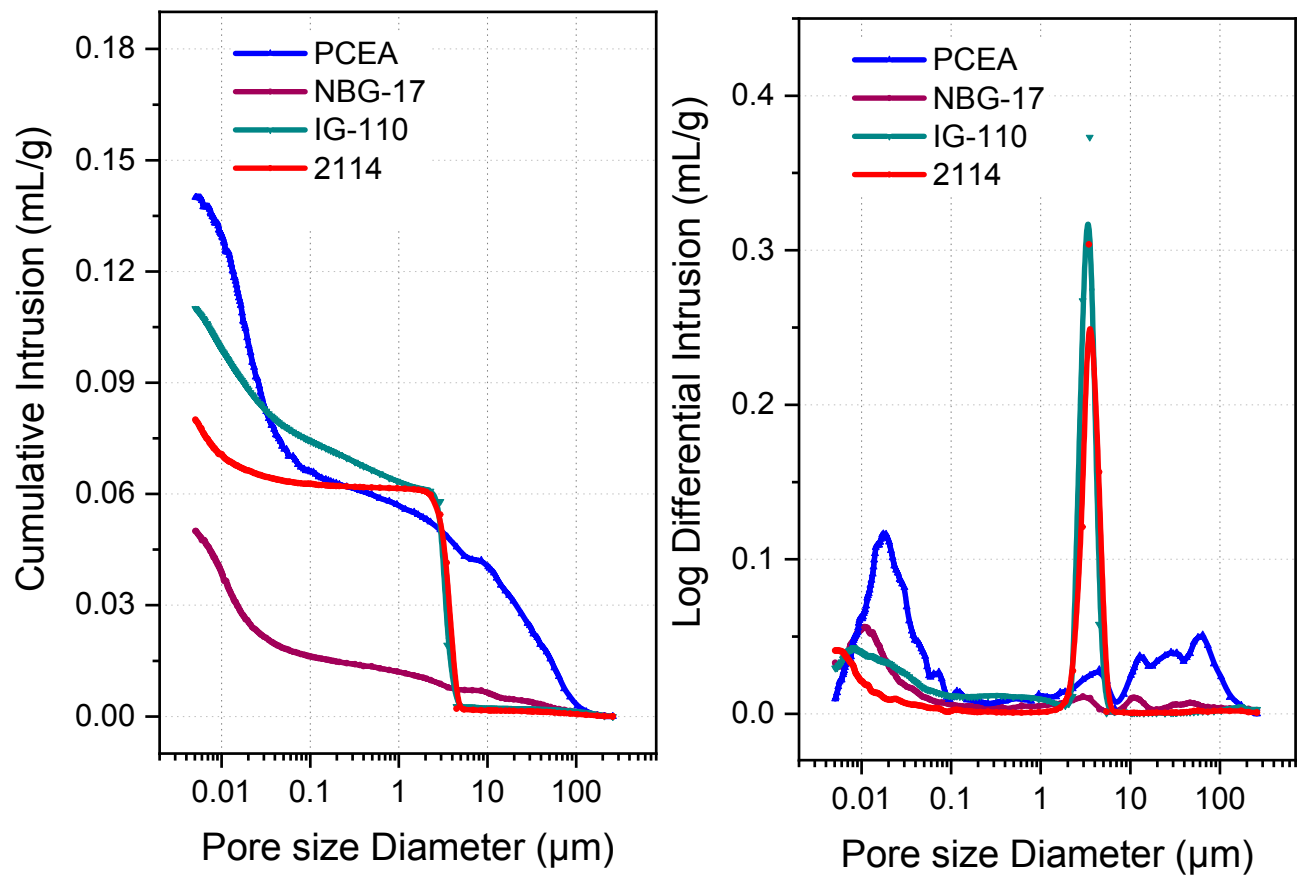

Figure 3. Pore size distribution from mercury intrusion porosimetry. 

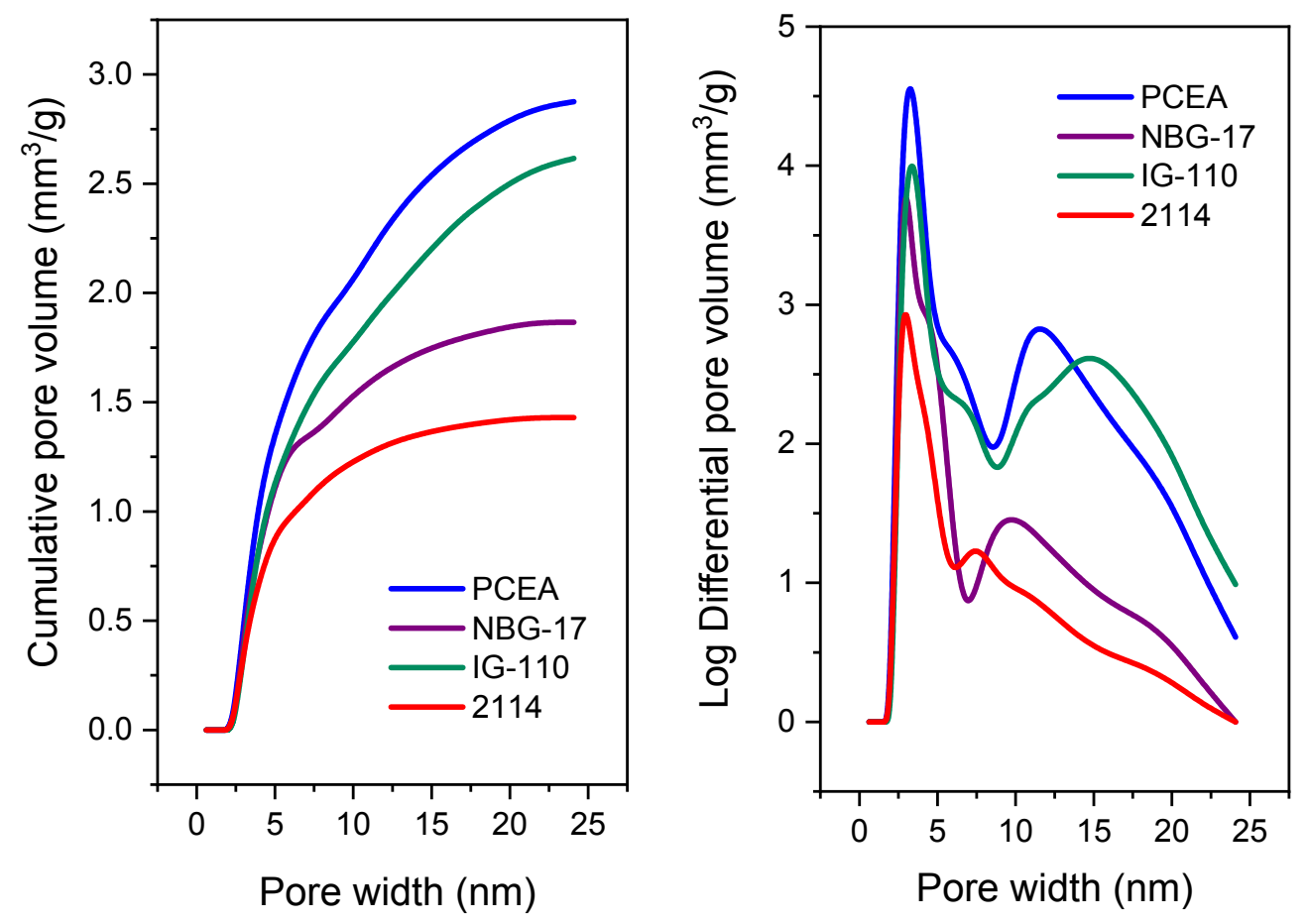

Figure 4. Pore size distribution from nitrogen adsorption measurements.

The pore structure of the four graphite grades was analyzed using mercury intrusion (Figure 3 ) and nitrogen adsorption (Figure 4). Mercury intrusion shows a broad distribution of pores in PCEA, broad distribution but less porosity in NBG-17, and narrow distributions around $3 \mu \mathrm{m}$ pores in IG-110 and 2114. Pores with widths $<0.1 \mu \mathrm{m}$ are also apparent from mercury data for all graphites. This might be because the presence of even narrower pores is confirmed by nitrogen adsorption (which reveals pores with sizes down to 3-4 nm). However, although pores with sizes between 5 and $24 \mathrm{~nm}$ are accessed by both mercury intrusion and gas adsorption, the cumulative volumes do not agree between the methods. Mercury intrusion shows larger total pore volumes than gas adsorption, which may indeed indicate that some damage was caused by high mercury pressures. Unlike mercury intrusion, gas adsorption does not change the material structure. It is interesting to observe that the cumulated mesopore volumes (between 3 and $25 \mathrm{~nm}$ ) increase in the order $2114<$ NBG-17 $<$ IG-110 $<$ PCEA.

\subsection{OUTLINE OF EXPERIMENTAL PROCEDURES AND VALIDATION STEPS}

The following is the short list of experimental steps pursued in this research and an outline of how the experimental results were integrated with Wichner's mathematical model.

- Measurements of oxidation rates were performed in accelerated conditions, covering a broad range of partial pressures of water vapor and hydrogen and a $300{ }^{\circ} \mathrm{C}$ interval of temperatures. The database collected for each grade of graphite was then analyzed statistically and the kinetic parameters were determined for each grade and tested against two different kinetic models.

- Water vapor transport through thin graphite samples was measured by a specialized testing lab. Measurements were made in the combined regime of permeation (pressure-driven) and diffusion 
(concentration-driven), and the results were used for evaluation of $D_{\text {eff }}$ and of specific structural differences of each graphite grade. Estimates of He permeability were also made.

- Samples oxidized at a weight loss larger than that obtained from kinetic measurements were produced for each grade of graphite at several levels of constant oxidation temperature. The oxidized layer penetration depth was evaluated by optical microscopy (and occasionally by x-ray tomography) from examination of transversal sections of oxidized samples.

- Excel spreadsheets were developed to correlate kinetic and transport information obtained previously. They were used for predicting oxidant penetration profiles as a function of the external conditions (water and hydrogen pressure, temperature) used for preparation of large-weight-loss specimens of each graphite grade.

- By comparing predicted penetration profiles against observed profiles of specimens oxidized at higher weight losses, conclusions were drawn about the validity and limitation of the Wichner model for chronic oxidation of nuclear graphite by moisture. 


\section{CHRONIC OXIDATION RATE MEASUREMENTS}

Measuring extremely slow oxidation rates is impractical. It may take years before any sizeable weight change would be observed for graphite exposed to very low partial pressures of water, as those projected for various HTGR designs. Information in Table 2 (adapted from ${ }^{32,33,34,35,36,37}$ ) summarizes the chemical environment and temperature conditions expected in He coolant during normal operation of HTGR.

Table 2. Chemical impurities in helium coolant and design temperatures in several HTGR projects.

\begin{tabular}{|l|c|c|c|c|c|c|c|c|c|}
\hline & \multicolumn{7}{|c|}{ Partial pressure (Pa) } & $\begin{array}{c}\text { Total } \\
\text { pressure }\end{array}$ & Temperature \\
\cline { 2 - 12 } & H2O & H2 & CO & O2 & N2 & CO2 & CH4 & (MPa) & in/out ('C) \\
\hline DRAGON, UK (1964-75) & 0.1 & 2 & 1.2 & $\mathrm{n} / \mathrm{a}$ & 0.3 & $<0.04$ & 0.3 & 2 & $350 / 750$ \\
\hline Peach Bottom, USA (1967-74) & 1.1 & 20.2 & 1.1 & $\mathrm{n} / \mathrm{a}$ & 1.5 & $<1.1$ & 2.2 & 2.25 & $377 / 750$ \\
\hline AVR, Germany (1967-88) & 3 & 30 & 10 & $\mathrm{n} / \mathrm{a}$ & $\mathrm{n} / \mathrm{a}$ & 10 & $\mathrm{n} / \mathrm{a}$ & 1.1 & $270 / 750-950$ \\
\hline Fort St. Vrain, USA (1976-79) & $<5$ & $10 . .35$ & $5 . .50$ & $\mathrm{n} / \mathrm{a}$ & $\mathrm{n} / \mathrm{a}$ & $2 . .14$ & $0.5 \ldots 4$ & 4.8 & $400 / 775$ \\
\hline HTR-10, China (2003) & $<1.4$ & $<21$ & $<21$ & $\mathrm{n} / \mathrm{a}$ & $<7$ & $\mathrm{n} / \mathrm{a}$ & $<7$ & 7 & $490 / 850$ \\
\hline HTR-PM, China (project) & 14 & 210 & 210 & 1.4 & 14 & 42 & 35 & 7 & $250 / 750$ \\
\hline HTTR, Japan (1998-present) & $<0.8$ & $<12$ & $<12$ & $\mathrm{n} / \mathrm{a}$ & $<0.8$ & $<2.4$ & $<2$ & 4 & $395 / 950$ \\
\hline NPH /HHT & 0.3 & 100 & 8 & $\mathrm{n} / \mathrm{a}$ & $1 . .2$ & $\mathrm{n} / \mathrm{a}$ & 10 & 0.2 & $\mathrm{n} / \mathrm{a} /<950$ \\
\hline PNP & 0.3 & 100 & 3 & $\mathrm{n} / \mathrm{a}$ & $<1$ & $\mathrm{n} / \mathrm{a}$ & 4 & 0.2 & $\mathrm{n} / \mathrm{a} /<950$ \\
\hline AGCNR & 0.4 & 80 & 8 & $\mathrm{n} / \mathrm{a}$ & $<4$ & 0.04 & 4 & 0.2 & $\mathrm{n} / \mathrm{a} /<950$ \\
\hline PBMR & 0.04 & 5 & 2 & $<0.01$ & $\mathrm{n} / \mathrm{a}$ & $\mathrm{n} / \mathrm{a}$ & $\mathrm{n} / \mathrm{a}$ & 9.0 & $400 / 900$ \\
\hline HTGR prismatic design & $0.7-1.4$ & $1.4-7.0$ & $1-3$ & $\mathrm{n} / \mathrm{a}$ & $<1.4$ & $<0.7$ & $<0.7$ & 7.0 & $540 / 900$ \\
\hline
\end{tabular}

Notes: NPH = nuclear power process heat; HHT = high-temperature helium turbine systems; $\mathrm{PNP}=$ prototype nuclear process heat; AGCNR = advanced gas-cooled nuclear reactor; PBMR = pebble bed modular reactor. Adapted from ref. ${ }^{24}$

For practical purposes, rather than attempt to reproduce in the laboratory these very low-humidity and high-temperature conditions, and then try to measure extremely slow oxidation rates, it is more efficient to perform accelerated oxidation tests in well-controlled and reproducible settings. Analysis of the results should lead to a general equation relating oxidation rates and the experimental variables (temperature, gas composition). After such a general model is found, the parameters specific to each material should be determined and used for estimations outside the envelope of experimentally measured values.

There are two caveats associated with this strategy. First, it is assumed that the same kinetic model applies in the laboratory conditions and in the out-of-reach conditions expected for HTGR normal operation. This is a reasonable assumption but is difficult to verify in most cases. Second, one should ensure that oxidation rate measurements are made, as much as possible, in diffusion-free conditions. That means that one should (1) perform measurements at temperatures at which diffusion rates are faster than chemical rates and (2) minimize diffusion paths by using graphite specimens with small dimensions. It may be difficult to meet both requirements at the same time, so minor perturbations of kinetic measurements by diffusion may be expected in some experiments. Practice showed that rate measurements at low temperatures $\left(800-850^{\circ} \mathrm{C}\right)$ often produced scattered results. We took actions to increase the accuracy of these data (for example, by increasing the collection times) but some data at 800 ${ }^{\circ} \mathrm{C}$ remained unreliable by themselves and could only be interpreted marginally in the general context of all other results obtained at higher temperatures. The selection of specimen size and shape in our 
experiments was also carefully considered. In their seminal analysis of chronic oxidation kinetics of graphite H-451, Burnette and colleagues from General Atomics used thin (1.6 mm) graphite discs that allowed two-sided exposure of bulk graphite. ${ }^{38}$ In our experiments, we used graphite rods with a 4-6 mm diameter. The cylindrical shape was more convenient because it ensured uniform exposure to the gas and radial penetration of oxidant.

\subsection{EXPERIMENTAL SETUP}

The experimental setup used for chronic graphite oxidation by moisture is presented in detail elsewhere. ${ }^{24,29}$ The main components (Figure 5) are (1) the TAG furnace 16/18 (Setaram, France), a dual symmetrical thermogravimetric analyzer used for continuous weight loss measurements of graphite specimens in flowing $\mathrm{H}_{2} \mathrm{O} / \mathrm{H}_{2} / \mathrm{He}$ at controlled temperatures; (2) the gas delivery manifold, which produces desired gas mixtures and controls the flow rates of each component (dry $\mathrm{He}$, moist $\mathrm{He}$, or 1\% $\mathrm{H}_{2} / \mathrm{He}$ ); (3) a controlled-temperature water bath for He humidification; (4) the hygrometer that measures the total gas pressure and the partial pressure of $\mathrm{H}_{2} \mathrm{O}$ just before the inlet of the TAG furnace; (5) a LabView application for data collection and control that commands the settings of mass flow controllers and records the corresponding flow rates, pressures, and humidity values; and (6) the mass spectrometer (Pfeiffer GSD 320) used occasionally for gas analysis of the effluent from the oxidation chamber in TAG.

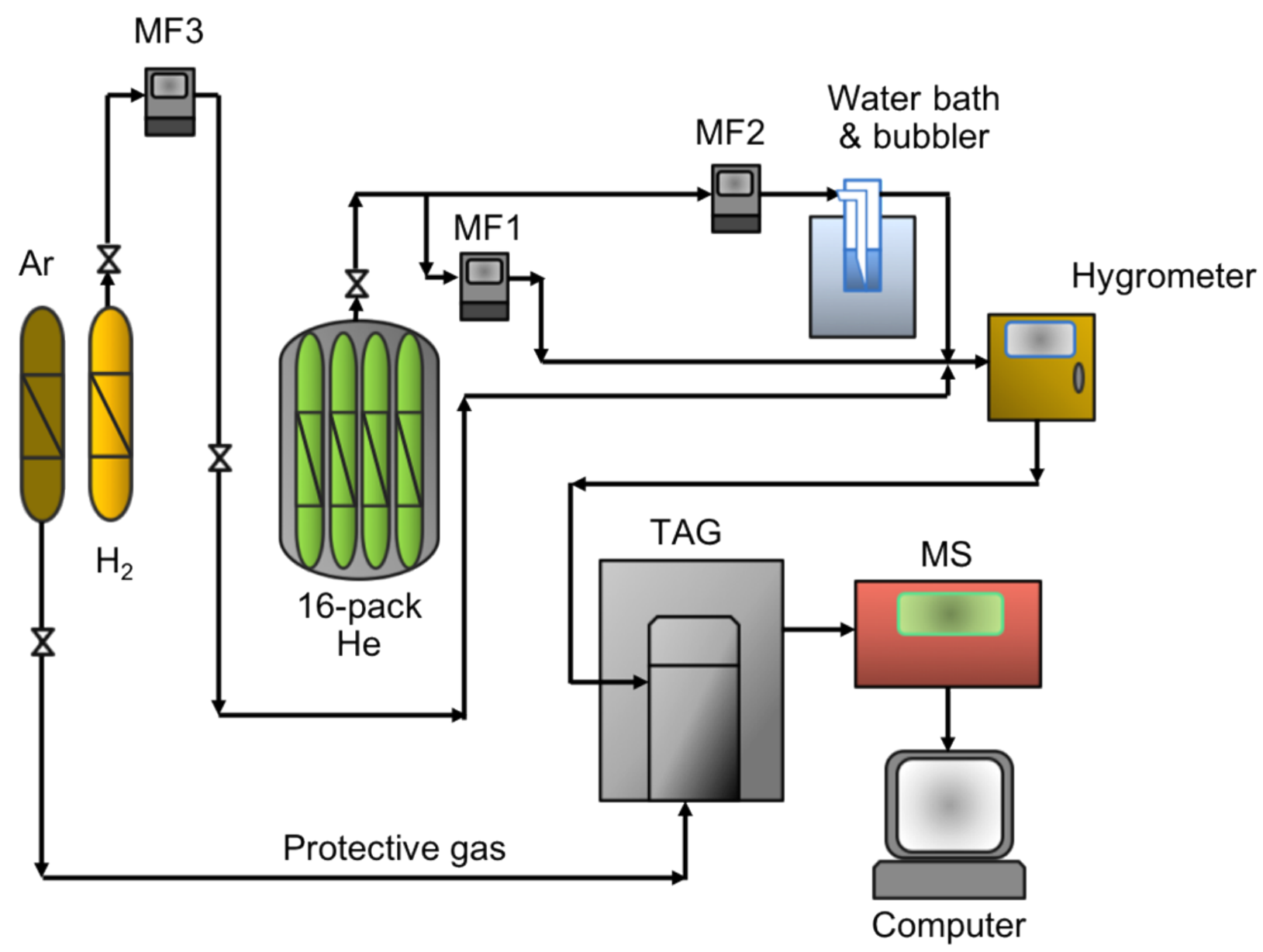

Figure 5. Schematic of experimental setup for chronic graphite oxidation by moisture. 


\subsection{DATA ACQUISITION}

In a typical experiment, a machined graphite rod, cleaned by ultrasonication in acetone and dried in air at $130{ }^{\circ} \mathrm{C}$, was weighed and its physical dimensions were recorded. The graphite rod was then attached with a platinum rod to the active arm of the TAG microbalance, and a quartz cylinder of similar volume was attached to the reference arm. After the specimen was outgassed at $1200{ }^{\circ} \mathrm{C}$ in flowing ultrahigh purity $\mathrm{He}$, the temperature was adjusted to the first segment in the experimental sequence. The flow of He was replaced by the gas composition with the desired values of partial pressures of water (and hydrogen in some experiments), $P_{\mathrm{H} 2 \mathrm{O}}$ and $P_{\mathrm{H} 2}$. With the gas composition fixed, the temperature was raised stepwise from the lowest to the highest in $50^{\circ} \mathrm{C}$ increments. At each level, the temperature was held constant for 3-5 $\mathrm{h}$ and the weight losses of the graphite specimens were continuously recorded. A complete experiment had 6-7 constant temperature steps between 800 and $1100^{\circ} \mathrm{C}$. Fresh specimens were used for each gas composition. Oxidation rates at each temperature were calculated from the rate of weight loss normalized by the actual weight at the beginning of each constant temperature segment (units $\mathrm{mg} \cdot \mathrm{mg}^{-1} \cdot \mathrm{s}^{-1}$ or $\mathrm{s}^{-1}$ ) Physical measurements were repeated for oxidized specimens at the end of the cycle. A full cycle with 6-7 temperature segments would take about 48-50 hours. The final weight loss of each specimen after a complete cycle was not larger than $1 \%$. Based on that, it was assumed that the oxidation was in the chemical control regime, the measurements were essentially not affected by diffusion, and the porosity changes, if any, were minimal. All gases were ultrahigh-purity grade, and the water for steam generation was ultrahigh-purity plasma grade.

Table 3 shows a summary of graphite specimens' properties and of experimental conditions used for each graphite grade. All specimens were cylindrical in shape and equal in size (except for the 2114 samples, which were slightly larger). They were placed coaxially in the center of the TAG furnace tube with a $21.5 \mathrm{~mm}$ ID. The gas flow direction was from top to bottom, at $1.5 \mathrm{~L} / \mathrm{min}$ volumetric flow rate (room temperature and pressure) or $4.03 \times 10^{-6} \mathrm{~kg} / \mathrm{s}$. The Reynolds number for this condition was less than 15 , which corresponds to laminar flow.

Table 3. Graphite specimen properties and range of experimental conditions for each grade

\begin{tabular}{|c|c|c|c|c|c|c|c|c|c|}
\hline \multirow[b]{2}{*}{ Grade } & \multicolumn{2}{|c|}{ Average size } & \multirow{2}{*}{$\begin{array}{c}\text { Average } \\
\text { weight } \\
\text { mg }\end{array}$} & \multicolumn{4}{|c|}{ Range of experimental conditions } & \multirow{2}{*}{$\begin{array}{c}\text { Total } \\
\text { specimens } \\
\text { tested }\end{array}$} & \multirow{2}{*}{$\begin{array}{c}\text { Total } \\
\text { data } \\
\text { points }\end{array}$} \\
\hline & $\begin{array}{c}\text { Diameter } \\
\mathbf{m m}\end{array}$ & $\begin{array}{c}\text { Length } \\
\mathrm{mm}\end{array}$ & & $\begin{array}{c}\text { Temperature } \\
{ }^{\circ} \mathrm{C} \\
\end{array}$ & $\begin{array}{c}P_{\text {H2O }} \\
\mathbf{P a} \\
\end{array}$ & $\begin{array}{c}P_{\mathrm{H} 2} \\
\mathbf{P a}\end{array}$ & $\begin{array}{c}\text { Flow rate } \\
\text { L/min }\end{array}$ & & \\
\hline PCEA & 4.01 & 20.00 & 445.9 & $850-1100$ & $3-300$ & $0-150$ & 1.5 & 103 & 355 \\
\hline NBG-17 & 3.99 & 20.01 & 463.8 & $800-1100$ & $3-1000$ & $0-100$ & 1.5 & 34 & 304 \\
\hline IG-110 & 4.04 & 20.08 & 455.5 & $850-1100$ & $5-1000$ & $0-100$ & 1.5 & 46 & 311 \\
\hline 2114 & 5.00 & 24.99 & 895.2 & $800-1100$ & $3-650$ & $0-90$ & 1.5 & 42 & 287 \\
\hline
\end{tabular}

Several hundred oxidation rate data were collected for each graphite grade by varying the experimental conditions over a broad range, as shown in Table 3. A small number of observations were rejected because of experimental errors, which were more frequent at low $P_{\mathrm{H} 2 \mathrm{O}}$ and low $T$ because very small weight variations challenged the microbalance resolution (which was very high, $0.5 \mu \mathrm{g}$ ). The total number of valid observations analyzed for each grade is shown in the last column of Table 3 . All numeric data were provided in table format in previous reports. ${ }^{24,26,28}$

\subsection{DATA ANALYSIS}

All valid data were analyzed by simultaneously solving a set of multiple nonlinear rate equations. The most likely parameter values were found by minimizing the sum of squared errors (SSE) for $Y=\log$ 
(Rate) where $Y$ is the rate measured at any given condition and Rate is calculated using a particular kinetic model. Two different models were used, as presented below.

\subsubsection{Classical Langmuir-Hinshelwood Model}

The chemical reaction that leads to chronic oxidation by moisture of nuclear graphite can be represented by the following global scheme:

$$
\mathrm{C}_{\text {graphite }}+\mathrm{H}_{2} \mathrm{O}_{\text {vapor }} \rightarrow \mathrm{CO}_{\text {gas }}+\mathrm{H}_{2} \text { gas }
$$

(Scheme I)

The exact sequence of elementary steps in the global process is not known. Several detailed mechanisms have been proposed, which differ in the way in which the site blocking role of hydrogen is representedeither in molecular form ${ }^{39}$ or in a chemisorbed atomic state. ${ }^{40}$ Each representation leads, however, to the same rate equation, which is the classical LH model:1,38

$$
\text { Rate }_{L H}\left(P_{H 2 O}, P_{H 2}, T\right)=\frac{K_{1} P_{H 2 O}}{1+K_{2}\left(P_{H 2}\right)^{0.5}+K_{3} P_{H 2 O}}
$$

where

$$
K_{i}=k_{i} \exp \left(-\frac{E_{i}}{R T}\right)
$$

In this equation, Rate is the weight-normalized oxidation rate $\left(\mathrm{mg}_{\text {oxidized }} / \mathrm{mg}_{\text {initial }} / \mathrm{s}\right.$ or $\left.\mathrm{s}^{-1}\right), T$ is the temperature $(\mathrm{K}), E_{i}$ is the activation energy $\left(\mathrm{J} \mathrm{mol}^{-1}\right)$ for the process $i$, and $R$ is the gas constant $(8.314 \mathrm{~J}$ $\left.\mathrm{mol}^{-1} \mathrm{~K}^{-1}\right)$. There are three kinetic constants $\left(K_{i} ; i=1,2,3\right)$ that contain a total of six parameters that must be estimated simultaneously by fitting all experimental data: three preexponential factors $\left(k_{i} ; i=1,2,3\right)$ and three apparent activation energies $\left(E_{i} ; i=1,2,3\right)$. 

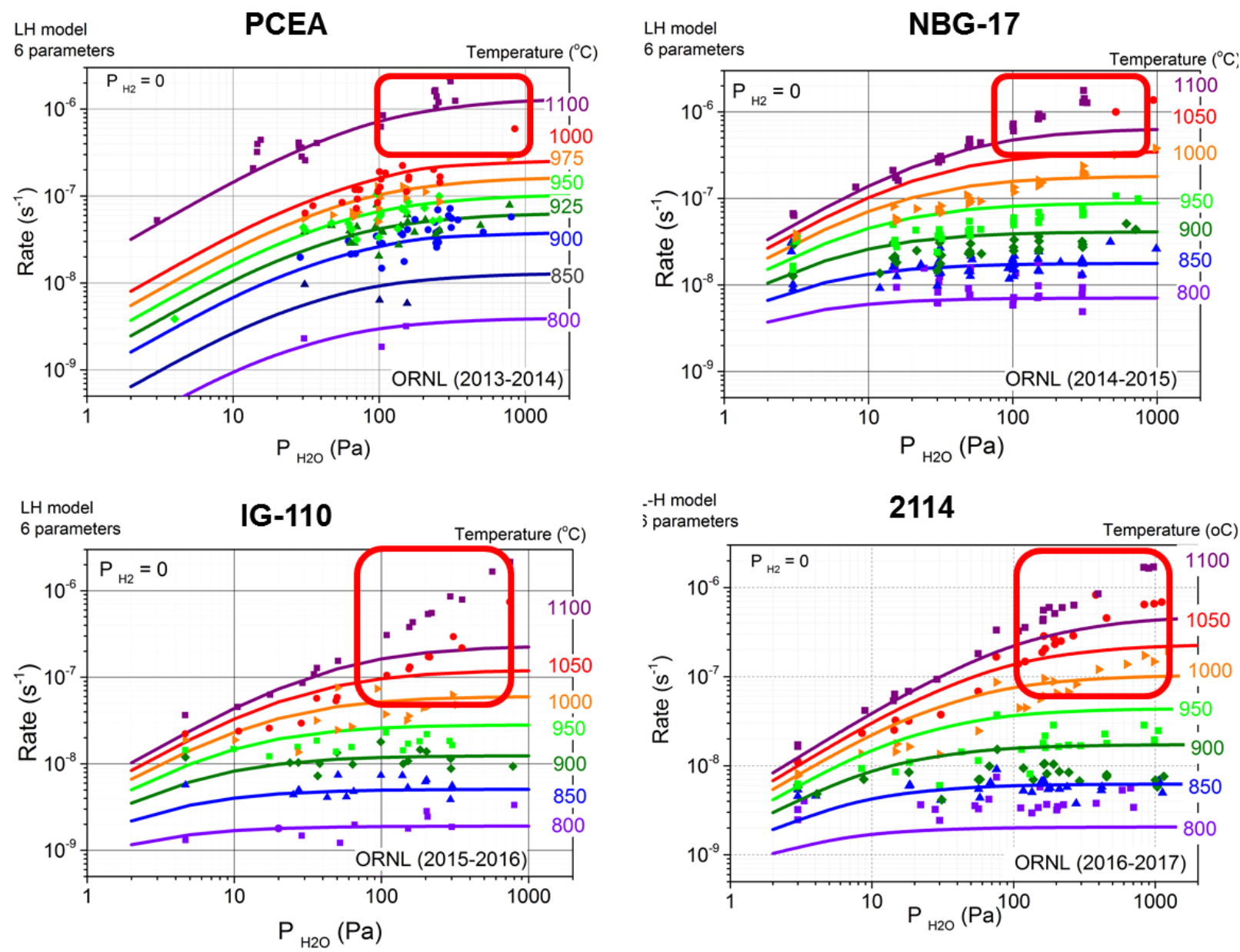

Figure 6. Experimental oxidation rate data (symbols) and their fitting by the LH kinetic model (continuous lines) at various temperatures. These plots show oxidation rates by water only $\left(\mathrm{P}_{\mathrm{H} 2}=0\right)$ in double logarithmic scale. Note the larger deviations for superfine grades IG-110 and 2114 when $\mathrm{P}_{\mathrm{H} 2 \mathrm{O}}>100 \mathrm{~Pa}$ and $\mathrm{T}>950{ }^{\circ} \mathrm{C}$.

The results of data analysis using the LH model are presented in Figure 6, where rate data obtained experimentally for each graphite grade are plotted versus $P_{\mathrm{H} 2 \mathrm{O}}$ on a double logarithmic scale. For simplicity, only data from experiments with no added $\mathrm{H}_{2}$ are shown in this figure. The continuous lines are predictions based on LH parameters found from fitting, which are provided in Table 4. Data and lines are color-coded for temperature.

Table 4. Langmuir-Hinshelwood analysis results for four graphite grades, showing temperature dependence of kinetic constants of Eq. (11).

\begin{tabular}{|c|c|c|c|c|}
\hline & PCEA & NBG-17 & IG-110 & 2114 \\
\hline$K_{1}$ & $5.9 \mathrm{E}-01 \times \exp (-198,700 / \mathrm{R} T)$ & $3.8 \mathrm{E}-06 \times \exp (-61,500 / \mathrm{R} T)$ & $8.3 \mathrm{E}-06 \times \exp (-85,800 / \mathrm{R} T)$ & $6.9 \mathrm{E}-07 \times \exp (-56,800 / \mathrm{R} T)$ \\
\hline$K_{2}$ & $5.4 \mathrm{E}+09 \times \exp (-279,500 / \mathrm{R} T)$ & $4.0 \mathrm{E}-08 \times \exp (186,600 / \mathrm{R} T)$ & $4.2 \mathrm{E}-08 \times \exp (193,200 / \mathrm{R} T)$ & $2.6 \mathrm{E}-02 \times \exp (23,900 / \mathrm{R} T)$ \\
\hline$K_{3}$ & $4.0 \mathrm{E}-04 \times \exp (39,000 / \mathrm{RT})$ & $5.8 \mathrm{E}-07 \times \exp (122,800 / \mathrm{R} T)$ & $6.3 \mathrm{E}-11 \times \exp (210,500 / \mathrm{R} T)$ & $4.9 \mathrm{E}-09 \times \exp (167,200 / \mathrm{R} T)$ \\
\hline$n$ & 0.5 & 0.5 & 0.5 & 0.5 \\
\hline
\end{tabular}

Note: $\mathrm{R}=8.314 \mathrm{~J} \mathrm{~mol}^{-1} \mathrm{~K}^{-1} ; T$ is absolute temperature in $\mathrm{K}$. The units of pre-exponent factors $k_{1}, k_{2}$, and $k_{3}$ are $\mathrm{Pa}^{-1} \mathrm{~s}^{-1} ; \mathrm{Pa}^{-0.5} ;$ and $\mathrm{Pa}^{-1}$ respectively; $n$ is the apparent kinetic order for hydrogen (no units). 
Examination of Figure 6 shows good fitting at low water vapor partial pressures $\left(P_{\mathrm{H} 2 \mathrm{O}}<100 \mathrm{~Pa}\right)$ and low to moderate temperatures $\left(\mathrm{T}<950^{\circ} \mathrm{C}\right)$. However, above these limits, the LH model was not capable of fitting the experimental observations. Oxidation rates increased faster with water vapor pressure than the LH model predicted. Differences were observed for all four graphite grades, but deviations were larger for superfine grain graphites (IG-110, 2114) than for medium-fine grain graphites (PCEA and NBG-17).

\subsubsection{Boltzmann-enhanced Langmuir-Hinshelwood Model}

To better fit all experimental data, we developed a new kinetic model. ${ }^{27,28,29}$ Its basic equation is derived from the LH model, except that a temperature-dependent kinetic order $m(T)$ for water was introduced, which replaced the $m=1$ value of the LH model. With that, the chemical reaction becomes

$$
\mathrm{C}_{\text {graphite }}+m \mathrm{H}_{2} \mathrm{O}_{\text {vapor }}=m \mathrm{CO}_{\text {gas }}+m \mathrm{H}_{2} \text { gas }+(1-m) \mathrm{C}_{\text {graphite }}
$$

(Scheme II)

Experiments have shown that $m(T)$ has a sigmoid variation with temperature, which was fitted well by the Boltzmann integral distribution function. Thus, the basic equation of the BLH model is

$$
\text { Rate }_{B L H}\left(P_{H 2 O}, P_{H 2}, T\right)=\frac{K_{1}\left(P_{H 2 O}\right)^{m(T)}}{1+K_{2}\left(P_{H 2}\right)^{0.5}+K_{3}\left(P_{H 2 O}\right)^{m(T)}},
$$

where

$$
K_{i}=k_{i} \exp \left(-\frac{E_{i}}{R T}\right) \text { and } m(T)=m_{\max }-\frac{m_{\max }-m_{\min }}{1+\exp \left(\frac{T-T_{o}}{\theta}\right)} .
$$

The new BLH kinetic model has ten parameters (three pre-exponential factors $A_{i}$, three activation energies $E_{i}$, the upper $\left(m_{\max }\right)$ and lower $\left(m_{\min }\right)$ limits of water apparent kinetic order, plus two more temperature parameters: $T_{0}$ which is a characteristic temperature associated with the inflection of $m(T)$ function, and $\theta$ which is a scaling parameter equal to the inverse slope of $m(T)$ at $T_{0}$. Using a similar procedure, the ten parameters were found by simultaneously fitting all experimental data. As Figure 7 shows, fitting with the BLH model substantially improved for all data, and the deviations at high temperatures and pressures disappeared. The graphite-specific BLH parameters are provided in Table 5. 

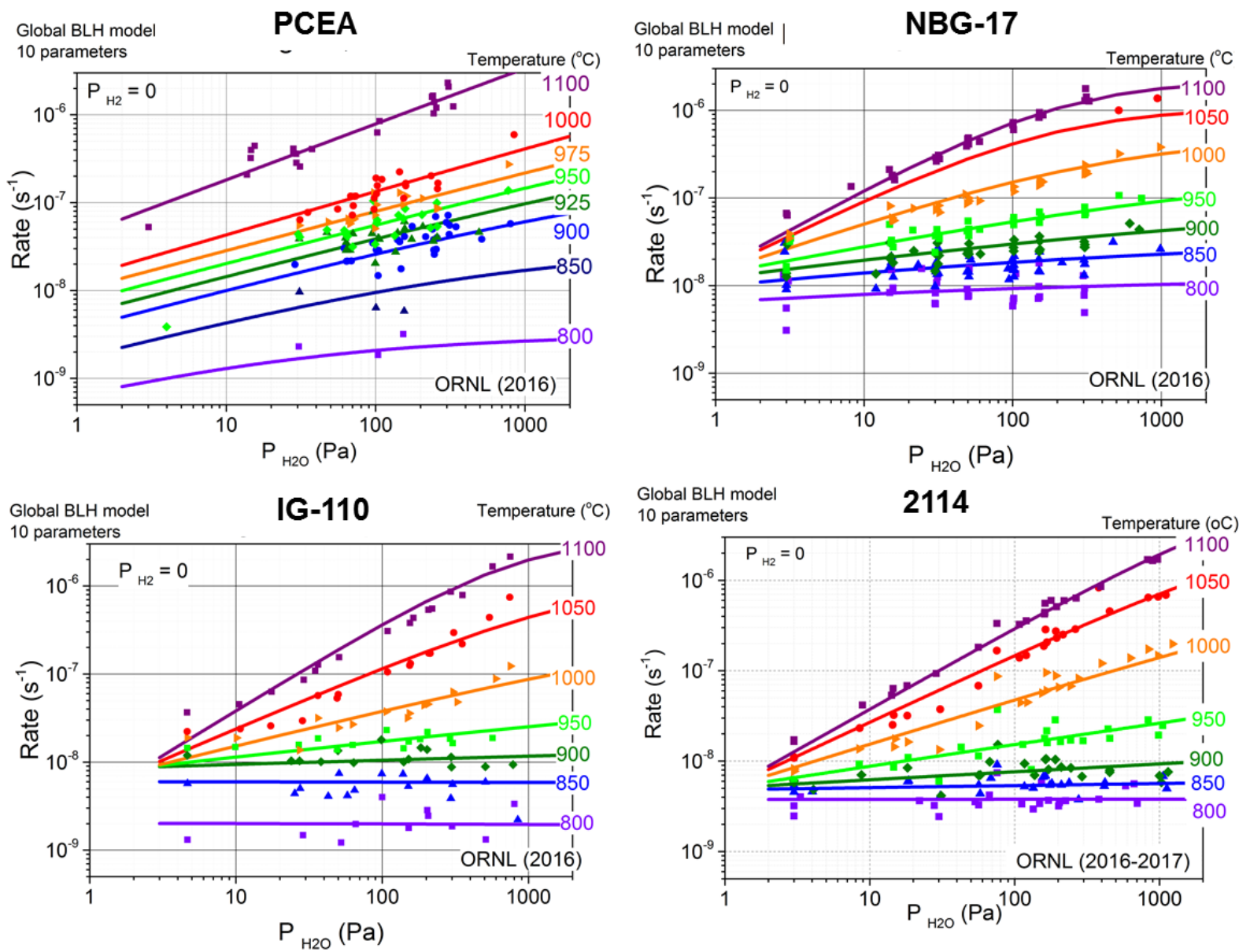

Figure 7. Experimental oxidation rate data (symbols) and their fitting by the new BLH kinetic model (continuous lines) at various temperatures. These plots show oxidation rates by water only $\left(\mathrm{P}_{\mathrm{H} 2}=0\right)$ in double logarithmic scale. Note the improved fitting for all grades in comparison with the LH model.

Table 5. Boltzmann-modified Langmuir-Hinshelwood analysis results of four graphite grades, showing temperature dependence of kinetic constants in Eq. (12).

\begin{tabular}{|c|c|c|c|l|}
\hline & PCEA & NBG-17 & IG-110 & \multicolumn{1}{c|}{2114} \\
\hline$K_{1}$ & $5.9 \mathrm{E}-02 \times \exp (-161,700 / \mathrm{R} T)$ & $4.3 \mathrm{E}-08 \times \exp (-11,400 / \mathrm{R} T)$ & $7.6 \mathrm{E}-12 \times \exp (70,600 / \mathrm{R} T)$ & $9.1 \mathrm{E}-12 \times \exp (61,000 / \mathrm{R} T)$ \\
\hline$K_{2}$ & $2.1 \mathrm{E}+05 \times \exp (-166,800 / \mathrm{R} T)$ & $3.9 \mathrm{E}-06 \times \exp (121,700 / \mathrm{R} T)$ & $1.9 \mathrm{E}-02 \times \exp (39,600 / \mathrm{R} T)$ & $1.5 \mathrm{E}+00 \times \exp (-1,650 / \mathrm{R} T)$ \\
\hline$K_{3}$ & $1.4 \mathrm{E}-15 \times \exp (292,600 / \mathrm{R} T)$ & $1.2 \mathrm{E}-10 \times \exp (203,200 / \mathrm{R} T)$ & $6.1 \mathrm{E}-18 \times \exp (373,500 / \mathrm{R} T)$ & $3.4 \mathrm{E}-14 \times \exp (280,600 / \mathrm{R} T)$ \\
\hline$m$ & $\begin{array}{l}0.64-0.19 /[1+\exp ((T- \\
1283) / 10.8)]\end{array}$ & $\begin{array}{l}0.93-0.70 /[1+\exp ((T- \\
1275) / 32.5)]\end{array}$ & $\begin{array}{l}1.55-1.62 /[1+\exp ((T- \\
1327) / 64.2)]\end{array}$ & $\begin{array}{l}1.46-1.40 /[1+\exp ((T- \\
1288) / 39.8)]\end{array}$ \\
\hline$n$ & 0.5 & 0.5 & 0.5 & 0.5 \\
\hline
\end{tabular}

Note: $\mathrm{R}=8.314 \mathrm{~J} \cdot \mathrm{mol}^{-1} \mathrm{~K}^{-1}$ and $T$ is the absolute temperature in $\mathrm{K}$. The units of pre-exponent factors $k_{1}, k_{2}$, and $k_{3}$ are $\mathrm{Pa}^{-m} \mathrm{~S}^{-1} ; \mathrm{Pa}^{-0.5} ;$ and $\mathrm{Pa}^{-m}$ respectively; $m$ is the apparent kinetic order for water (no units). 


\subsubsection{Comparison of LH and BLH Models}

The fitting quality obtained from the two kinetic models can be appreciated by comparing log-log plots of measured data versus predicted (fitted) data in Figure 8 and Figure 9.
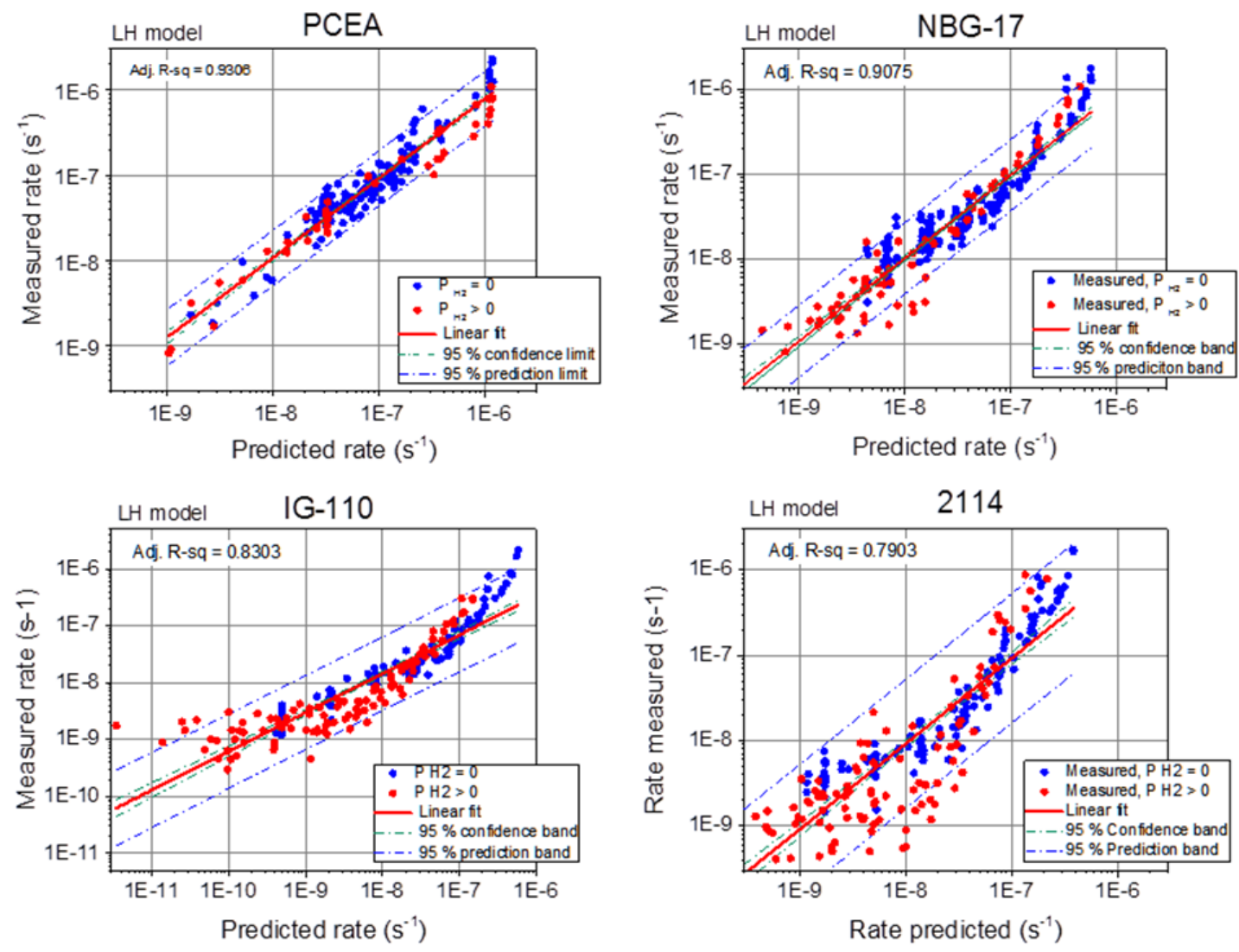

Figure 8. Comparison of fitting quality by using the LH model with parameters from Table 4. All data are shown in blue for $\mathrm{P}_{\mathrm{H} 2}=0$ and in red for $\mathrm{P}_{\mathrm{H} 2}>0$ 

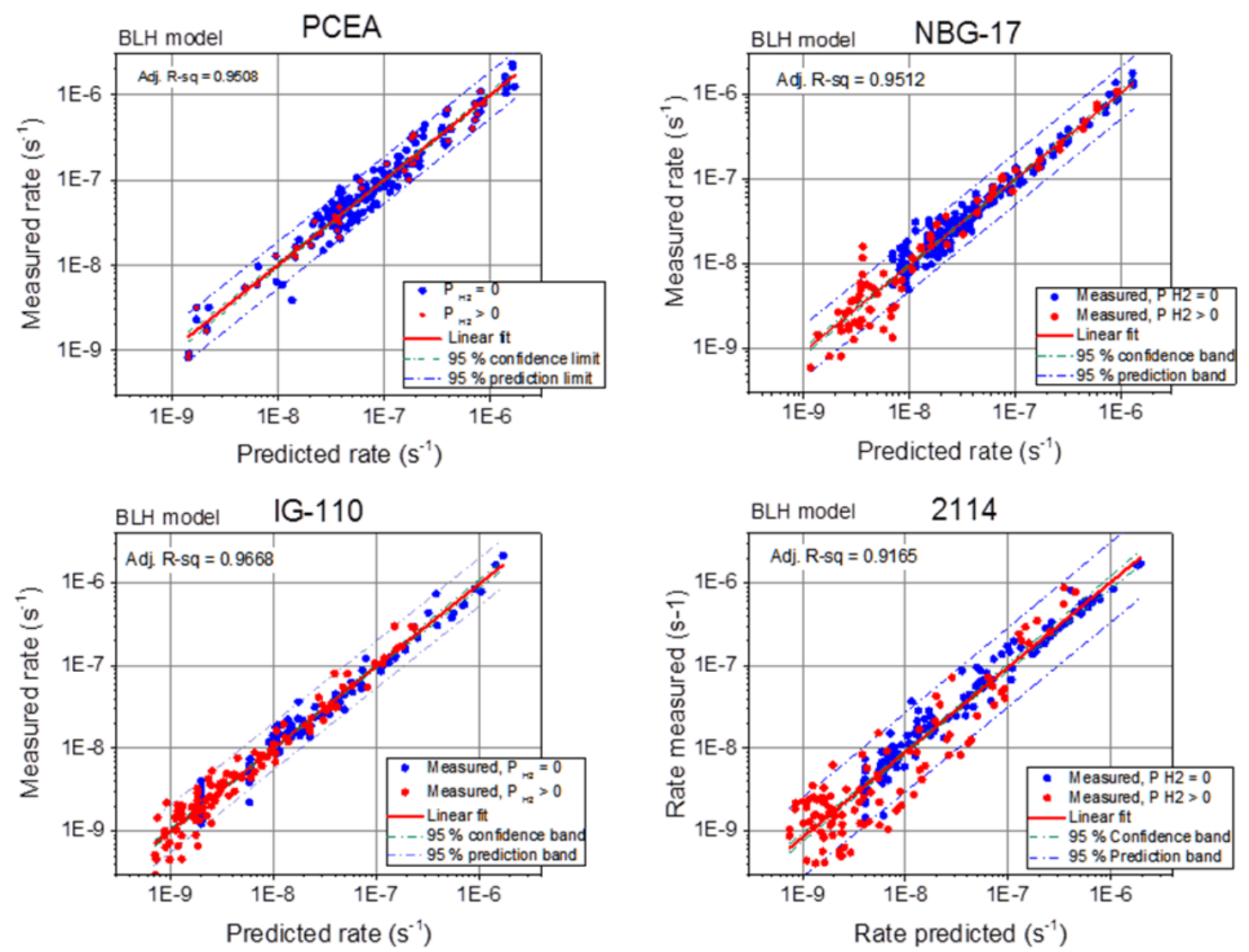

Figure 9. Comparison of fitting quality by using the BLH model with parameters from Table 5. All data are shown in blue for $\mathrm{P}_{\mathrm{H} 2}=0$ and in red for $\mathrm{P}_{\mathrm{H} 2}>0$

From these two figures, it is seen that, in general, the BLH model achieves a better fit than the LH model for all oxidation rate data over about four orders of magnitude. The same conclusion is obtained by comparing statistical parameters describing the fitting quality between the two models for each graphite grade (Table 6). It is seen that global fitting of data (with and without $\mathrm{H}_{2}$, all temperatures) is better for medium grain grades (PCEA and NBG-17) and is worst for the superfine grain graphite 2114.

Table 6. Fitting quality parameters for LH and BLH models compared between graphite grades.

\begin{tabular}{|l|cc|cc|cc|cc|}
\hline & \multicolumn{2}{|c|}{ PCEA } & \multicolumn{2}{c|}{ NBG-17 } & \multicolumn{2}{c|}{ IG-110 } & \multicolumn{2}{c|}{2114} \\
\cline { 2 - 10 } & LH & BLH & LH & BLH & LH & BLH & LH & BLH \\
\hline \hline Residual sum of squares & 4.92 & 3.49 & 12.09 & 6.38 & 22.11 & 4.32 & 38.00 & 21.12 \\
Pearson's r & 0.97 & 0.98 & 0.95 & 0.98 & 0.91 & 0.98 & 0.89 & 0.95 \\
Adjusted R-square & 0.93 & 0.95 & 0.91 & 0.95 & 0.83 & 0.97 & 0.79 & 0.90 \\
\hline
\end{tabular}




\section{WATER VAPOR TRANSPORT MEASUREMENTS}

\subsection{BACKGROUND}

The second component of the Wichner model is the oxidant transport through the porous structure of graphite. Water vapor transport occurs in two ways: permeation driven by pressure differences, and diffusion driven by concentration differences. The terminology in the discussion below follows the recent review by Webb. ${ }^{41}$

\subsubsection{Gas Permeation through Porous Media}

Forced permeation in porous media follows Darcy's law:

$$
w=\frac{Q}{A}=v \varepsilon=\left(\frac{\kappa}{\mu}\right)\left(\frac{\Delta P}{L}\right),
$$

where $w \quad=$ Darcy velocity, $\mathrm{m} / \mathrm{s}$

$Q \quad=$ volumetric gas flow rate through the porous medium, $\mathrm{m}^{3} / \mathrm{s}$

$A=$ cross section area for transport in the porous medium, $\mathrm{m}^{2}$

$\kappa \quad=$ gas-phase permeability, $\mathrm{m}^{2}$

$\mu \quad=$ gas viscosity, Pa.s

$\Delta P \quad=$ applied pressure difference, $\mathrm{Pa}$

$L \quad=$ thickness of porous medium, $\mathrm{m}$

$v \quad=$ gas velocity in pores, $\mathrm{m} / \mathrm{s}$

$\varepsilon \quad=$ volume fraction of transport pores

If the gas flow velocity is expressed in mass units, the equivalent form of Eq. (13) is

$$
F_{\text {perm }}=\frac{\dot{M}}{A}=J \Delta P=j \frac{\Delta P}{L}
$$

where $F_{\text {perm }}$ = permeation mass flux (area-normalized mass transmission rate) of gas, $\mathrm{kg} \cdot \mathrm{m}^{-2} \cdot \mathrm{s}^{-1}$

$\dot{M} \quad=$ mass flow rate, $\mathrm{kg} / \mathrm{s}$

$A \quad=$ cross section area of the porous medium, $\mathrm{m}^{2}$

$J \quad=$ gas permeance (dependent on specific experimental conditions), $\mathrm{kg} \cdot \mathrm{m}^{-2} \cdot \mathrm{s}^{-1} \cdot \mathrm{Pa}^{-1}$

$j \quad=$ mass permeability coefficient, $\mathrm{kg} \cdot \mathrm{m}^{-1} \cdot \mathrm{s}^{-1} \cdot \mathrm{Pa}^{-1}$

$L \quad=$ thickness of porous medium, $\mathrm{m}$

The mass permeability coefficient $j$ in Eq. (14) is related to medium absolute permeability $\kappa$ from Eq. (13) through gas density, $\rho_{\text {gas }}$, and viscosity, $\mu$ :

$$
j=\frac{\kappa}{\mu} \rho_{\text {gas }} .
$$

\subsubsection{Gas Diffusion through Porous Media}

In conditions of negligible gas-pore wall interactions (i.e., in pores larger than the mean free path of gas molecules), viscous (normal) diffusion is driven by concentration (or partial pressure) differences. Knudsen diffusion becomes important at extreme conditions (narrow pores, low pressure) when the mean free path becomes larger or comparable with the pore size. Water molecules and He atoms have similar 
van der Waals diameters $\left(d_{\mathrm{H} 2 \mathrm{O}}=0.282 \mathrm{~nm} ; d_{\mathrm{He}}=0.280 \mathrm{~nm}\right)$ and almost equal mean free paths $\left(\lambda_{\mathrm{H} 2 \mathrm{O}}=119\right.$ $\left.\mathrm{nm} ; \lambda_{\mathrm{He}}=121 \mathrm{~nm}\right)$ at the laboratory conditions of effective diffusivity measurements $\left(35^{\circ} \mathrm{C}, 1 \mathrm{bar}\right.$ total pressure). Thus, Knudsen diffusion is expected in pores with sizes $<120 \mathrm{~nm}$. Mercury porosimetry (Figure 3) and nitrogen adsorption (Figure 4) indicate that such pores occur in all graphites. However, the path for normal (viscous) diffusion in larger pores is more than half of the total open pore volume for IG110 and 2114, almost half for PCEA (49 \%), and less for NBG-17 (30\%).

The effect of Knudsen diffusion is not much different in the conditions of oxidation rate measurements. The mean free paths increase with the temperature, up to about $420 \mathrm{~nm}$ at $800{ }^{\circ} \mathrm{C}$ and $530 \mathrm{~nm}$ at $1100{ }^{\circ} \mathrm{C}$ at 1 bar total pressure. However, the pore size distributions of the four graphites are almost flat between $0.15 \mu \mathrm{m}$ and $0.55 \mu \mathrm{m}$, so that the fraction of pore volumes open for viscous diffusion does not change much with the increase of temperature. Therefore, the water transport measurements at laboratory conditions can still be used for evaluation of effective diffusivity at the temperatures of oxidation measurements (with neglect of Knudsen diffusion as a first approximation).

To account for the diffusion in porous media, it is customary to use the effective diffusion coefficient defined as

$$
D_{e f f}=\beta D_{A B},
$$

where $\beta$ is a structural factor specific to each grade, and $D_{A B}$ is the binary diffusion coefficient in bulk $\mathrm{AB}$ gas mixture (no porous media). The SI unit for $D_{\text {eff }}$ and $D_{A B}$ is $\mathrm{m} / \mathrm{s}^{2}$. According to the molecular gas theory, $D_{A B}$ varies with temperature and total pressure as

$$
D_{A B}(P, T)=D_{A B}\left(P_{0}, T_{0}\right)\left(\frac{T}{T_{0}}\right)^{q P_{0}} \frac{1}{P}
$$

where normally $q=1.5$. However, for $\mathrm{H}_{2} \mathrm{O}-\mathrm{He}$ mixtures, the exact value of $q$ may be different, given the special properties of water. The Landolt-Bornstein handbook gives $q=1.75$, based on a report by Fuller et al. ${ }^{42}$ Newer experimental data and analysis of older reports corrected the exponent value ${ }^{43}$ to $q=1.338$. This value is used for correlations in the following chapters.

The porous media correction factor $\beta$ depends on microstructure properties, such as porosity fraction, constriction factor in pore connectivity, and pore tortuosity. Several correlations, ${ }^{41,44,45,46,47}$ not always in agreement with one another, have been proposed. Rather than analyzing them, which is not the scope of this report, we focused on measuring the effective diffusivity for $\mathrm{H}_{2} \mathrm{O}$ from $\mathrm{H}_{2} \mathrm{O} / \mathrm{He}$ mixtures for each grade of graphite. The temperature of all measurements was $35^{\circ} \mathrm{C}$, and the pressure was the daily atmospheric pressure. The measurements used a system in which permeation and diffusion occurred simultaneously.

\subsubsection{Simultaneous Permeation and Diffusion}

There are several models for combined diffusion and permeation transport in porous media. The ADM (advective-diffusive model) is the linear addition of Darcy's permeation law and Fick's diffusion law. ${ }^{41}$ Porous medium effects are included through the structural factor, $\beta$, applied to the diffusion flux in free space; see Eq. Deff $=\beta D_{A B},(16)$ above. Equation (18) (with Knudsen diffusion and gravitational effects neglected) holds only for combined permeation and diffusion in the viscous flow regime:

$$
F=-\frac{\kappa}{\mu} \rho_{g a s} \frac{\Delta P}{L}-\rho D_{\text {eff }} \frac{\Delta x}{L},
$$




where $\begin{aligned} F & =\text { mass flux, } \mathrm{kg} \cdot \mathrm{m}^{-2} \cdot \mathrm{s}^{-1} \\ \kappa & =\text { permeability, } \mathrm{m}^{2} \\ \mu & =\text { gas viscosity, } \mathrm{Pa} \cdot \mathrm{s} \\ \rho_{\text {gas }} & =\text { gas density, } \mathrm{kg} / \mathrm{m}^{3} \\ \Delta P & =\text { gas pressure difference across the specimen, } \mathrm{Pa} \\ \Delta x & =\text { water concentration difference across the specimen (molar fractions) } \\ L & =\text { specimen thickness, } \mathrm{m} \\ D_{\text {eff }} & =\text { effective diffusivity in the porous system of component } \mathrm{A} \text { in } \mathrm{AB} \text { gas mixture, } \mathrm{m}^{2} / \mathrm{s}\end{aligned}$

The ADM model ignores the coupling between permeation and diffusion. If needed, Knudsen diffusion may be included as a correction factor for gas permeability, $\kappa$. The dusty gas model (DGM) includes explicitly the coupling between different diffusion mechanisms. However, the ADM and DGM models agree with each other only for materials with moderate permeability $\left(>10^{-13} \mathrm{~m}^{2}\right)$ and if the total pressure is 1 bar. $^{42}$ If the material's permeability is lower (e.g., $10^{-18} \mathrm{~m}^{2}$ ) Fick's law overpredicts the gas diffusion flux at 1 bar total pressure. The neglect of Knudsen diffusion in the ADM model makes a negligible difference for medium-permeability materials $\left(10^{-12} \mathrm{~m}^{2}\right)$ and represents a small correction factor $(0.5-2.5)$ for lower-permeability materials $\left(10^{-18} \mathrm{~m}^{2}\right)$.

Theoretically, in a system with regular and Knudsen diffusion, the global diffusion coefficient is calculated ${ }^{48}$ as

$$
\frac{1}{D_{\text {global }}}=\frac{1}{D_{e f f}}+\frac{1}{D_{k}}
$$

where $D_{\text {eff }}=\beta D_{\mathrm{AB}}$ as above and $D_{K}$ is the Knudsen diffusion coefficient. For a material with idealized structure (parallel capillaries) the Knudsen diffusion coefficient can be estimated as

$$
D_{k} \cong 97 r_{\text {pore }} \sqrt{\frac{M_{w}}{T}},
$$

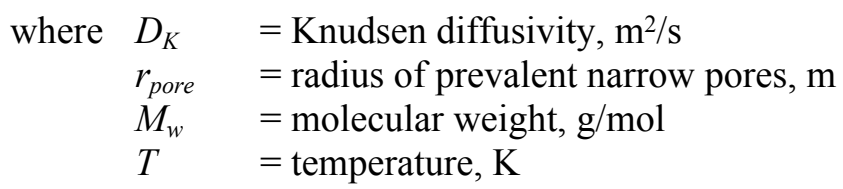

We later use these formulae in an attempt to verify the effect of Knudsen diffusivity on the penetration depth of an oxidized layer in graphites IG-110 and 2114.

\subsection{EXPERIMENTAL SETUP}

Measurements of water vapor transport were performed by Porous Materials Inc. (PMI), an independent contract testing laboratory in Ithaca, NY, and a leader in porosity solutions. ${ }^{49}$ The procedure was adapted after the ASTM F2298, the standard test method for measurement of moisture vapor transmission rates and gas flow properties through fabrics, membranes, laminates, and other materials. ${ }^{2}$ The water vapor transmission analyzer was equipped with a dynamic moisture permeation cell (DMPC) described below.

\footnotetext{
${ }^{2}$ ASTM F2298-03, Standard test method for water vapor diffusion resistance and air flow resistance of clothing materials using the dynamic moisture permeation cell (reapproved 2009).
} 


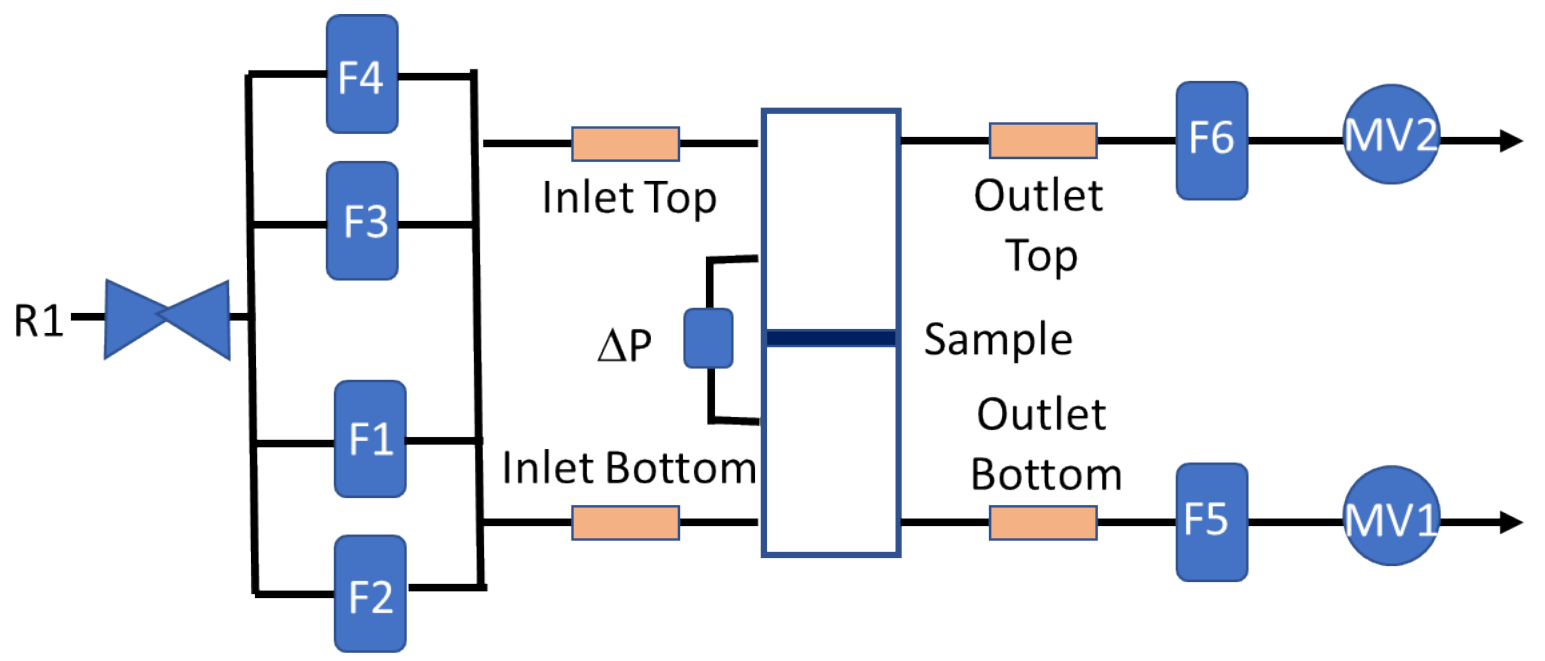

Figure 10. Schematic of DMPC instrument used for measurements of water vapor transmission rates. $^{3}$

A DMPC is a modern version of a Wicke cell ${ }^{50}$ used for studies on surface diffusion, and for diffusion measurements in porous systems. ${ }^{51,52,53}$ (Figure 10, adapted from DMPC User Manual ${ }^{3}$ ). A thin graphite slab is sealed in a temperature-controlled cell. Two independent gas streams flow on each side of the specimen and communicate only by gas transport through the sample's porosity. A gas regulator (R1 in the figure) allows variable amounts of $\mathrm{He}$ gas to enter the cell. A fraction of the gas stream on either side of the sample goes through water bubblers while the remainder or the gas stream is kept dry. By adjusting the flow rate of dry and wet sub-streams on each side of the sample, one controls the inlet gas humidity of each stream. The humidity and temperature on each side of the cell are measured on the inlet and outlet lines. There are four humidity sensors (represented as orange rectangles in the figure) on each side of the sample cell and on each gas line. Four temperature sensors are located near the respective humidity sensors, inside the sample chamber. The outlet gas flow rate is measured by flow meters on the top and bottom sides of the cell (F5 and F6). Two motor valves at the outlet ends of gas streams (MV1 and MV2) control the differential pressure between the top and bottom lines, which is recorded by the differential pressure gauge $(\Delta \mathrm{P})$. The sample holder and all other instrumentation are maintained at constant temperature, controlled by a heating/chilling auxiliary system. The instrument is built with high-accuracy electronic sensors and controllers. Calibration conditions are $0{ }^{\circ} \mathrm{C}$ and 760 Torr. Corrections for actual temperature and pressure are applied by instrument software. The operation procedure and data analysis routine are prescribed by ASTM F2298. ${ }^{49}$

\subsection{DATA COLLECTION AND RESULTS}

Figure 11 shows schematically the sequence of operations in the DMPC. While the differential pressure of the He gas is varied between $\Delta P<0$ through $\Delta P=0$ and finally to $\Delta P>0$, the relative humidity levels in the top and lower gas lines are maintained constant. Thus, water transport between the top and bottom compartments is helped (Figure 11 a) or hindered (Figure 11c) by the pressure-driven water permeation. A neutral situation occurs at $\Delta P=0$ (Figure 11b) when water transport is caused solely by the relative humidity (concentration) difference between the top and bottom lines. In practice, it is difficult to maintain this singular situation and accurately measure water vapor transport rate. Rather than using approximate conditions more or less near the $\Delta P=0$ point, the transmission rate at $\Delta P=0$ was calculated by linear regression of the experimental data when $\Delta P$ varied from negative to positive values.

\footnotetext{
${ }^{3}$ Water vapor transmission analyzer (DMPC) user's manual. Porous Materials Inc. (2014)
} 


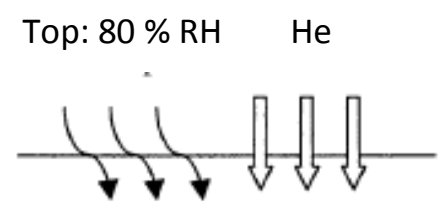

Bottom: $15 \% \mathrm{RH}$

Negative He pressure gradient

(a)

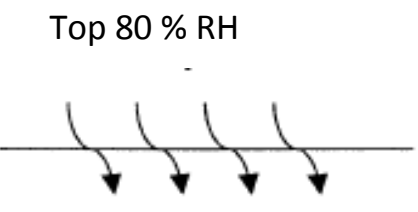

Bottom $15 \% \mathrm{RH}$

Zero pressure gradient
Top: $80 \% \mathrm{RH}$

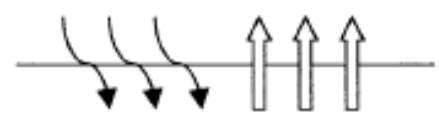

Bottom: $15 \% \mathrm{RH} \quad \mathrm{He}$

Positive He pressure gradient

(c)

Figure 11. Schematic of test sequences in DMPC: (a) and (c) combined diffusion and permeation of water vapor and helium permeation; (b) water diffusion only, no He flow.

Water transport properties were measured in two different instances. The first tests with graphite grades PCEA and NBG-17 were performed in 2015 and a preliminary report was issued. ${ }^{25} \mathrm{~A}$ second series of tests done in 2017-2018 included all four graphite grades.

Figure 12 shows experimental results plotted accorded to Eq. (18) for two different graphite specimen orientations ( $\mathrm{WG}=$ with grain; $\mathrm{AG}=$ against grain). The mas flow at $\Delta P=0$ was obtained from linear fitting of the transmission rate versus the pressure difference. The effective diffusivity was then calculated from known values of the water molar fraction difference $(\Delta x)$ between the top and bottom gas lines and the graphite slab thickness $(L)$. The gas density value $\rho_{\text {gas }}$ in Eq. (18) was replaced by the logarithmic mean water vapor concentration difference ${ }^{50}$ between the top and bottom gas lines, $\Delta C$ :

$$
\Delta C=\frac{\Delta C_{a}-\Delta C_{b}}{\ln \left(\Delta C_{a} / \Delta C_{b}\right)},
$$

where $\Delta C_{a}$ and $\Delta C_{b}$ are the water vapor concentration difference between the two He streams at the incoming end (a) or outgoing end (b) of the flow cell, respectively, in $\mathrm{kg} / \mathrm{m}^{3}$. 

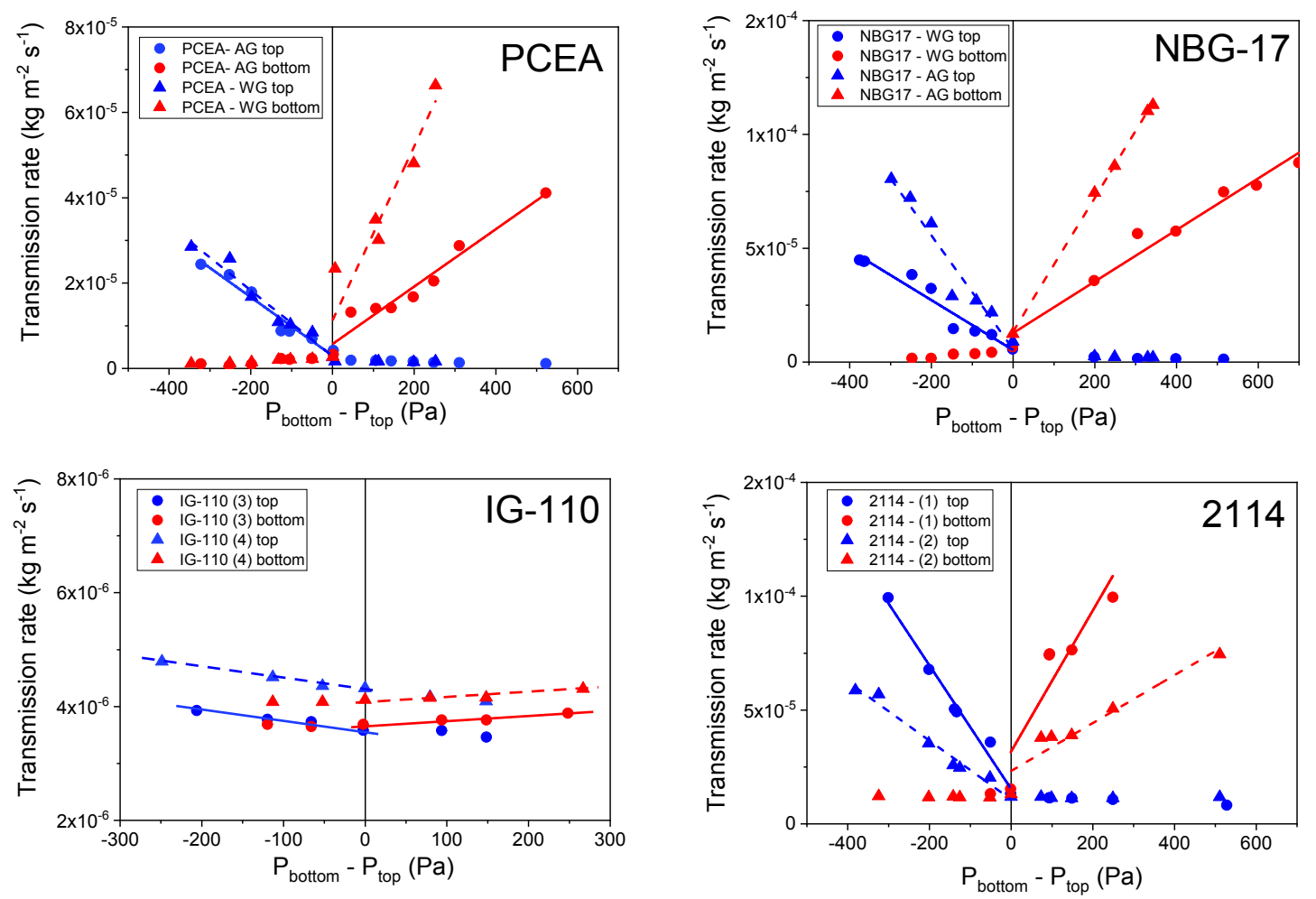

Figure 12. Experimental results showing water transmission rates as a function of pressure difference across the graphite specimens.

Table 7 presents experimental details for specimens and measurement conditions, along with the calculated $D_{\text {eff }}$ from individual measurements. It also shows small orientation-dependent $D_{\text {eff }}$ values for the near-isotropic graphite grades PCEA and NBG-17. The structural factor $\beta$ introduced by Eq. (16) was calculated in each experiment by comparing the $D_{\text {eff }}$ calculated from water transmission rates in each experiment with the actual $D_{\mathrm{H} 2 \mathrm{O}}$ in $\mathrm{H}_{2} \mathrm{O} / \mathrm{He}$ mixtures at the actual experiment conditions. For that approach, the reference value for $D_{H 2 O}\left(0.908 \mathrm{~cm}^{2} / \mathrm{s}\right.$ at $25^{\circ} \mathrm{C}$ and 760 Torr) reported by Lee and Wilke was recalculated for the actual temperature $\left(35^{\circ} \mathrm{C}\right)$ and barometric pressure at the time of the measurements using Eq. (17) and Luijten's value $q=1.338$ for the exponent. ${ }^{43}$ The last column in Table 7 shows the average values of the structural factors for each graphite grade. 
Table 7. Experimental details for water transport measurements and effective diffusivity results

\begin{tabular}{|c|c|c|c|c|c|c|c|c|c|c|}
\hline \multirow{2}{*}{ Grade } & \multirow{2}{*}{ Campaign } & \multirow{2}{*}{ Orientation } & \multirow{2}{*}{$\begin{array}{c}\text { Thickness } \\
\mathrm{cm}\end{array}$} & \multirow{2}{*}{$\begin{array}{c}\text { Area } \\
\mathrm{cm}^{2}\end{array}$} & \multirow{2}{*}{$\begin{array}{c}\text { Average } D_{\text {eff }} \\
\text { top \& bottom }\end{array}$} & \multirow{2}{*}{$\begin{array}{c}\text { Actual } D_{\mathrm{H} 2 \mathrm{O}} \\
\text { free gas }\end{array}$} & \multirow{2}{*}{$\frac{\text { Struct factor }}{\beta \times 10^{3}}$} & \multicolumn{2}{|c|}{ Average $\beta \times 10^{3}$} & \multirow{2}{*}{$\frac{\text { He permeability }}{\mathbf{m}^{2}}$} \\
\hline & & & & & & & & Orientation & General & \\
\hline \multirow{6}{*}{ PCEA } & \multirow{2}{*}{2014} & \multirow{3}{*}{ WG } & 0.151 & 8.70 & $3.06 \mathrm{E}-03$ & 0.910 & $3.37 \pm 0.43$ & \multirow{3}{*}{$3.49 \pm 0.69$} & \multirow{6}{*}{$2.83 \pm 0.51$} & \multirow{6}{*}{$7 \mathrm{E}-13$} \\
\hline & & & 0.144 & 8.70 & $3.57 \mathrm{E}-03$ & 0.910 & $3.93 \pm 0.18$ & & & \\
\hline & & & 0.105 & 9.85 & $2.90 \mathrm{E}-03$ & 0.912 & $3.18 \pm 1.45$ & & & \\
\hline & 2011 & \multirow{3}{*}{$\mathrm{AG}$} & 0.099 & 9.75 & $1.68 \mathrm{E}-03$ & 0.906 & $1.85 \pm 0.41$ & \multirow{3}{*}{$2.17 \pm 0.33$} & & \\
\hline & \multirow{2}{*}{2014} & & 0.168 & 8.70 & $1.91 \mathrm{E}-03$ & 0.910 & $2.10 \pm 0.24$ & & & \\
\hline & & & 0.158 & 8.70 & $2.32 \mathrm{E}-03$ & 0.910 & $2.56 \pm 0.34$ & & & \\
\hline \multirow{6}{*}{ NBG-17 } & \multirow{2}{*}{2014} & \multirow{3}{*}{ WG } & 0.168 & 8.70 & $6.59 \mathrm{E}-04$ & 0.910 & $0.72 \pm 0.23$ & \multirow{3}{*}{$1.89 \pm 0.63$} & \multirow{6}{*}{$2.09 \pm 0.64$} & \multirow{6}{*}{$4 \mathrm{E}-14$} \\
\hline & & & 0.167 & 8.70 & 7.54E-04 & 0.910 & $0.83 \pm 0.13$ & & & \\
\hline & 2017 & & 0.100 & 9.75 & $3.73 \mathrm{E}-03$ & 0.909 & $4.11 \pm 1.55$ & & & \\
\hline & 2011 & \multirow{3}{*}{$\mathrm{AG}$} & 0.102 & 9.75 & $4.24 \mathrm{E}-03$ & 0.912 & $4.66 \pm 1.55$ & \multirow{3}{*}{$2.29 \pm 0.65$} & & \\
\hline & \multirow{2}{*}{2014} & & 0.165 & 8.70 & $9.48 \mathrm{E}-04$ & 0.910 & $1.04 \pm 0.18$ & & & \\
\hline & & & 0.162 & 8.70 & $1.07 \mathrm{E}-03$ & 0.910 & $1.17 \pm 0.23$ & & & \\
\hline \multirow{4}{*}{ IG-110 } & \multirow{4}{*}{2017} & \multirow{4}{*}{ Isotropic } & 0.104 & 9.97 & $2.51 \mathrm{E}-03$ & 0.912 & $2.75 \pm 0.42$ & \multirow{4}{*}{\multicolumn{2}{|c|}{$2.69 \pm 0.21$}} & \multirow{4}{*}{$6.5 \mathrm{E}-15$} \\
\hline & & & 0.105 & 9.57 & $2.68 \mathrm{E}-03$ & 0.912 & $2.94 \pm 0.42$ & & & \\
\hline & & & 0.151 & 9.73 & $2.16 \mathrm{E}-03$ & 0.918 & $2.35 \pm 0.41$ & & & \\
\hline & & & 0.151 & 9.71 & $2.48 \mathrm{E}-03$ & 0.912 & $2.72 \pm 0.01$ & & & \\
\hline \multirow{2}{*}{2114} & 2017 & Isotronic & 0.099 & 9.70 & $9.45 \mathrm{E}-03$ & 0.915 & $10.3 \pm 0.41$ & 010 & & $6 \mathrm{E}$ \\
\hline & 2011 & Isotropic & 0.105 & 9.70 & $7.17 \mathrm{E}-03$ & 0.909 & $7.89 \pm 0.42$ & 9.10 & 42 & $6 \mathrm{E}-13$ \\
\hline
\end{tabular}


The graphite-specific structural factors $(\beta)$ defined in Eq. $\quad$ Deff $=\beta D_{A B}, \quad(16)$ are plotted in Figure 13 as a function of the apparent density and the total porosity fraction of the four graphites. This figure contains ORNL data (with error bars) for $\mathrm{H}_{2} \mathrm{O}$ diffusivity from $\mathrm{H}_{2} \mathrm{O} / \mathrm{He}$ mixtures (Table 7) and two more sets of data measured at Idaho National Laboratory (INL) by Kane et al ${ }^{42}$ for the diffusivity of $\mathrm{N}_{2}$ from $\mathrm{N}_{2} /$ Ar mixtures and diffusivity of Ar from $\mathrm{N}_{2} /$ Ar mixtures through the same graphite grades. Like ORNL, INL used a type of Wicke cell ${ }^{50}$ but the data were collected by mass spectrometry. Remarkably, the results differ by a factor of not larger than 2 , which gives confidence in the accuracy of measurements. Recall that the higher the $\beta$ values in a series of graphites, the higher is $D_{\text {eff. }}$ Globally, it appears that the increase in apparent density (or total porosity fraction) in the series IG-110 < NBG-17 < PCEA correlates with a decline (increase) of structural factors and effective diffusivity. Graphite 2114 does not follow this trend. Kane et al. ${ }^{42}$ argued that the different behavior of grade 2114 reflects the pore structure. In general, if Knudsen diffusion occurs in parallel with regular (viscous) diffusion in bimodal pore structure, the effect of Knudsen diffusion is to lower the effective diffusivity. Thus, they argued, the higher $D_{\text {eff }}$ (or $\beta$ ) of grade 2114 suggests that Knudsen diffusion is less important for this graphite than for the other three grades. At the time of publication of their paper, Kane et al. ${ }^{44}$ did not have microstructural information in support of this assertion. Now, one can see from mercury porosimetry results (Figure 3 ) and gas adsorption data (Figure 4) that the cumulated mesopore volumes (between 3 and $25 \mathrm{~nm}$ ) increase in the order $2114<$ NBG-17 < IG-110 < PCEA. With the lowest amount of mesopore volume of all four grades and permeability as high as that of PCEA (Table 7), graphite 2114 has reduced Knudsen diffusion, and its effective diffusivity (for $\mathrm{H}_{2} \mathrm{O}, \mathrm{N}_{2}$ and $\mathrm{Ar}$ ) is the highest of all grades.
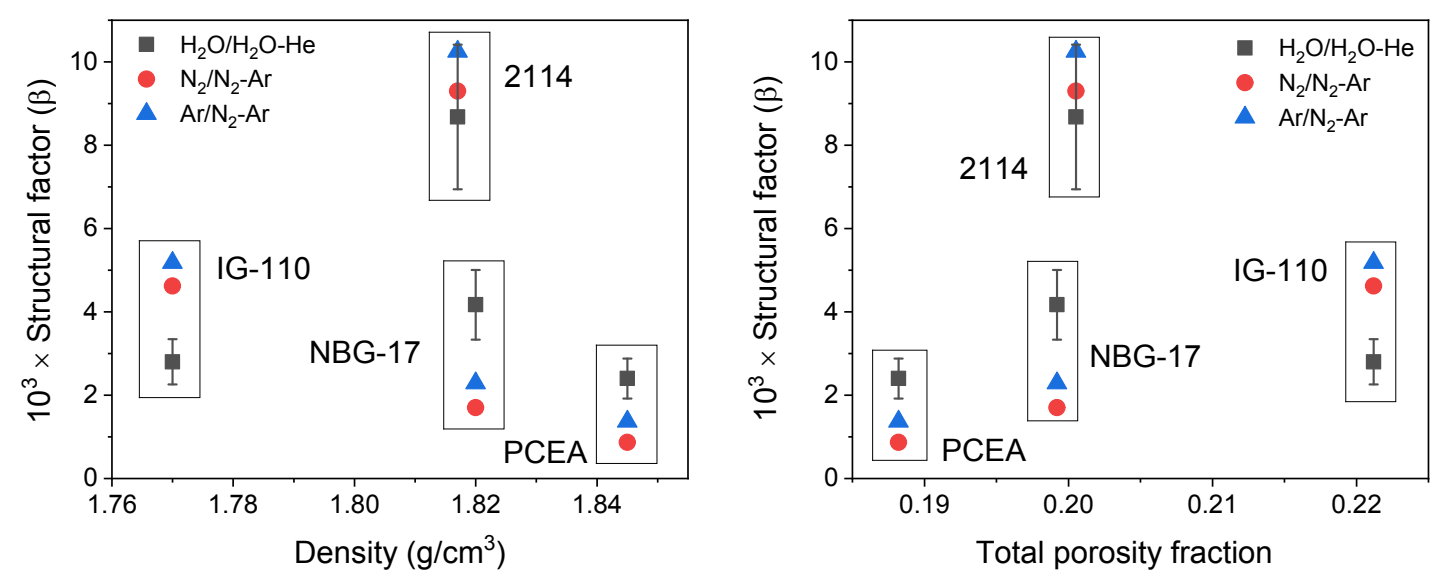

Figure 13. Comparing graphite structural factors measured from diffusion experiments at ORNL and INL versus graphite density and total porosity fraction.

There are two more competing temperature effects that may change the importance of Knudsen diffusion under the conditions of the oxidation experiments. First, the temperature dependence of diffusion coefficients is different: $D_{A B}$ varies with $T^{q}$ where $q=1.338$ for $\mathrm{H}_{2} \mathrm{O}$ in $\mathrm{He}^{43}$ in the viscous diffusion regime and $n=1 / 2$ for Knudsen diffusion. As Kane et al. calculated, the ratio of viscous to Knudsen diffusion should be more than 3.25 times larger at $700{ }^{\circ} \mathrm{C}$ than at room temperature. However, the mean free path also increases with temperature, and that contributes to adding more pores of larger diameter to the group of pores involved in Knudsen diffusion. The increase of mean free path probably would not be significant for medium-fine grain graphites (PCEA, NBG-17); but for superfine grain graphites (2114, IG-110), the importance of Knudsen diffusion may increase significantly at the temperatures of chronic 
oxidation. ${ }^{44}$ Further studies are needed to accurately quantify the Knudsen diffusion contribution at room temperature and at the oxidation temperatures.

It is shown later in this report that calculations of oxidized layer profiles with the effective diffusivity corrected by the approximative relations from Eqs. (19) and (20) returned better results for 2114 but did not significantly improve the agreement between predictions and observations for IG-110. 


\section{MODELING OXIDIZED LAYER DEVELOPMENT IN TRANSIENT CONDITIONS}

The information on oxidation kinetics and oxidant transport for each graphite grade available so far can be used for analyzing the development of the oxidized layer, following the Wichner model. However, a few more steps are needed to clarify the significance of various assumptions inherent to this model.

\subsection{SIMPLIFYING ASSUMPTIONS}

Some simplifying assumptions used in the Wichner model are justified by early observations of graphite oxidation (by air and steam) reported by various researchers. ${ }^{8,9,10}$ Others were introduced to minimize the mathematical burden on the model user. In addition, these simplifications ease the rapid survey of the effect of key parameters that control the oxidation process.

\subsubsection{Quasi-steady State}

In early stages of oxidation, the rate of inward diffusion of the oxidant slows as the rate of oxidant consumption in the chemical reaction accelerates. At some point in time, the two processes will find dynamic equilibrium, and from then oxidation will continue in quasi-steady state conditions. In fact, oxidation is never truly steady, since material is being continuously consumed and removed. However, once a fully developed concentration profile is stabilized, one may consider that oxidation progresses by slowly moving inward of a density profile with unaltered shape as long as the conditions of temperature, free gas composition, and pressure do not change. The time needed for stabilization of the fully developed oxidation profile is analyzed in a later section. It is shown that this time is short, and that the density profile and the maximum penetration of the oxidized layer depend only on temperature, through the combined effect of temperature on the rate of chemical reaction and on the rate of oxidant species transport through diffusion. Proof of the temperature dependence of the oxidation profile was obtained from oxidation by air and steam experiments. $8,9,10$

\subsubsection{Constant Effective Diffusivity}

Since the local density in the oxidized layer varies with the distance from the surface, $D_{\text {eff }}$ also varies with position because of its relationship with the microstructure details (e.g., porosity, density). In fact, the assumption of a constant $D_{\text {eff }}$ throughout the oxidized layer contradicts the assumption of a fully developed density profile. However, there are arguments that constant $D_{\text {eff }}$ in the oxidized layer is an acceptable simplification that yields results in agreement with density profile measurements in oxidized layers. ${ }^{5}$

\subsubsection{Linearized Oxidation Kinetics derived from the LH Model}

Each of the two kinetic models used for describing experimental observations has advantages and disadvantages. One would expect that the LH model would better fit experimental data at low $P_{\mathrm{H} 2 \mathrm{O}}$ and low $T$, and for that reason it should be the choice for extrapolating experimental data from accelerated oxidation tests back to the milder conditions of chronic oxidation during normal operation. The BLH model, on the other hand, provides more confidence in the range of high $P_{\mathrm{H} 2 \mathrm{O}}$ and $T$, going up to extreme conditions that might occur hypothetically in the case of steam ingress. Indeed, very recent measurements at ORNL with oxidation of fuel matrix carbon by water showed the BLH model predictions compare favorably with reaction rate values measured in simulated steam ingress conditions. ${ }^{55,56}$ Verification tests with nuclear graphite are necessary and should be done in the near future.

In a previous chapter, it was shown that the two models are in reasonably good agreement. Moreover, the linear equation derived from the LH model follows satisfactorily the experimental data between 800 and 
$1000{ }^{\circ} \mathrm{C}$ for all four graphite grades. This indicates that using the linearized equation may be accepted as a zero approximation because it is greatly advantageous from the computational point of view. In fact, meaningful linearized equations can only be derived from the LH model, by reducing Eq. (4) to a shorter, linear form:

$$
\text { Rate }_{L H-\text { linear }} \cong k_{1} \exp \left(-\frac{E_{1}}{R T}\right) P_{H 2 O} .
$$

In the case of the BLH model, replacing Eq. (12),

$$
\text { Rate }_{B L H}=\frac{k_{1} \exp \left(-\frac{E_{1}}{R T}\right) P_{H 2 O} m(T)}{1+k_{2} \exp \left(-\frac{E_{2}}{R T}\right)\left(P_{H 2}\right)^{n}+k_{3} \exp \left(-\frac{E_{3}}{R T}\right) P_{H 2 O} m(T)},
$$

with

$$
\text { Rate }_{B L H-l i n e a r} \cong k_{1} \exp \left(-\frac{E_{1}}{R T}\right) P_{H 2 O} m(T)
$$

is not meaningful because fitting parameters in the BLH model did often led to negative values for activation energies. With $E_{1}<0$ in Eq. (23), the linearized BLH rate would have the wrong temperature variation, despite that fact that the full BLH rate does correctly reproduce the experimental dependence.

\subsubsection{Ideal Gas Law Behavior}

Using the linearized LH form ( Eq. [22]) allows for analytical solutions of oxidation rates and density profiles in specimens with simple geometrical shapes. It also permits coupling of the surface graphite oxidant concentration with the oxidant pressure in the gas free stream by the use of ideal gas laws:

$$
\text { Rate }_{\text {linear }}(\vec{r}, t)=k_{u} \exp \left(\frac{-E_{u}}{\mathrm{R} T}\right) f(\alpha) \mathrm{RT} u(\vec{r}, t),
$$

where $u$ is the oxidant concentration of $\mathrm{H}_{2} \mathrm{O}$ in pores ( $\mathrm{mol} / \mathrm{m}^{3}$-void).

\subsubsection{Constant Graphite Density}

In the fully developed oxidized profile, the graphite density may vary from a remnant of $10-20 \%$ of the unoxidized density at the exposed surface, up to the unoxidized density in the interior. This variation changes the local porosity $(\varepsilon)$ in the oxidized layer and affects the effective diffusivity $\left(D_{e f f}\right)$. To account for that variation, Richards ${ }^{2}$ introduced a corrective factor $f(\alpha)$ derived from the random-pore model and expressed as a function of the weight loss level, In our experiments, the very low levels of weight loss justify neglecting the changes in porosity and diffusivity during oxidation.

\subsection{TRANSIENT SOLUTION}

Assume a semi-infinite slab with face at $x=0$ exposed to an oxidant in concentration $U\left(\mathrm{~mol} / \mathrm{m}^{3}\right)$ in the free space extending to infinity in the positive $x$ direction. The general equation for oxidant transport and consumption introduced earlier as Eq. (5),

$$
\partial \mathrm{u} \partial \mathrm{t}=\operatorname{Deff} \varepsilon \partial 2 \mathrm{u} \partial-\mathrm{x} 2-\mathrm{A} \varepsilon \mathrm{u},(5)
$$


led to the steady state solution when $t \rightarrow \infty$, Eq. (9),

$$
\mathrm{u}(\mathrm{x}) \mathrm{U}=\exp (--B x)
$$

where $A$ and $B$ were defined earlier by Eqs. (6) and (8). The same equation gives information on the time evolution of the oxidized layer profile. With new boundary conditions, $u(x, 0)=0$ (zero oxidant concentration at $t=0)$ and $u(0, t)=U$ (constant oxidant concentration, $U$, in the external gas phase), the new solution is ${ }^{5,6}$

$$
\frac{u(x, t)}{U}=\frac{1}{2} \exp (-x B) \operatorname{erfc}\left(A_{1}\right)+\frac{1}{2} \exp (x B) \operatorname{erfc}\left(A_{2}\right)
$$

where

$$
A_{1}=\frac{x}{2 \sqrt{\frac{D_{e f f} t}{\varepsilon}}}-\sqrt{\frac{A t}{\varepsilon}} \text { and } A_{2}=\frac{x}{2 \sqrt{\frac{D_{e f f}}{\varepsilon}}}+\sqrt{\frac{A t}{\varepsilon}}
$$

and $e r f c$ is the complementary error function. The partial pressure of the oxidant does not appear in Eq. (25) because, under the linearizing assumptions, the increased tendency to penetrate is compensated by the loss rate due to oxidation.

Since the kinetic and diffusivity parameters are known at this point for each graphite grade, Eq. (25) can be evaluated using $t$ as a variable and $X_{50 \%}$ as the distance below the surface where the concentration of the oxidant drops by $50 \%$. The parameters used for this calculation are shown in Table 8 . Calculations were performed for atmospheric pressure and several temperatures. The effective diffusivity $D_{\text {eff }}$ values were calculated using Eq. (16) and the structural factors $\beta$ indicated in Table 8 . The free gas diffusion coefficients for water in He were adjusted for temperature starting from $D_{\mathrm{H} 2 \mathrm{O}}=0.908 \mathrm{~cm}^{2} / \mathrm{s}$ at $25^{\circ} \mathrm{C}$ and 760 Torr $^{54}$ and Eq. (17) with $q=1.338 .{ }^{43}$ The results in Figure 14 show that the development of the fully stabilized density profile of the oxidized layer is significantly accelerated by temperature. The transient regime is short, only a few seconds, before the rate of inward oxidant diffusion is compensated by the rate of oxidant consumption in the chemical reaction. There are noticeable differences between graphite grades, determined by their kinetic rate parameters in the linearized LH equation and by their corresponding $\beta$ factors which are related to microstructure. 
Table 8. Structural and kinetic parameters used for estimation of time needed for development of a stable density profile.

\begin{tabular}{|l|l|c|c|c|c|}
\hline & & PCEA & NBG-17 & IG-110 & $\mathbf{2 1 1 4}$ \\
\hline \multirow{3}{*}{ Structure } & Apparent density $\rho, \mathrm{g} / \mathrm{cm}^{3}$ & 1.804 & 1.854 & 1.779 & 1.815 \\
\cline { 2 - 6 } & Structural factor, $\beta \times 10^{3}$ & 2.83 & 1.94 & 2.69 & 9.1 \\
\hline \multirow{3}{*}{ Kinetics } & Pre-exponential factor $k_{l}, \mathrm{~s}^{-1} \mathrm{~Pa}^{-1}$ & $5.9 \times 10^{-1}$ & $3.85 \times 10^{-6}$ & $8.29 \times 10^{-6}$ & $6.89 \times 10^{-7}$ \\
\cline { 2 - 7 } & Activation energy $E_{l}, \mathrm{~kJ} / \mathrm{mol}$ & 198.7 & 61.5 & 85.7 & 56.8 \\
\hline
\end{tabular}

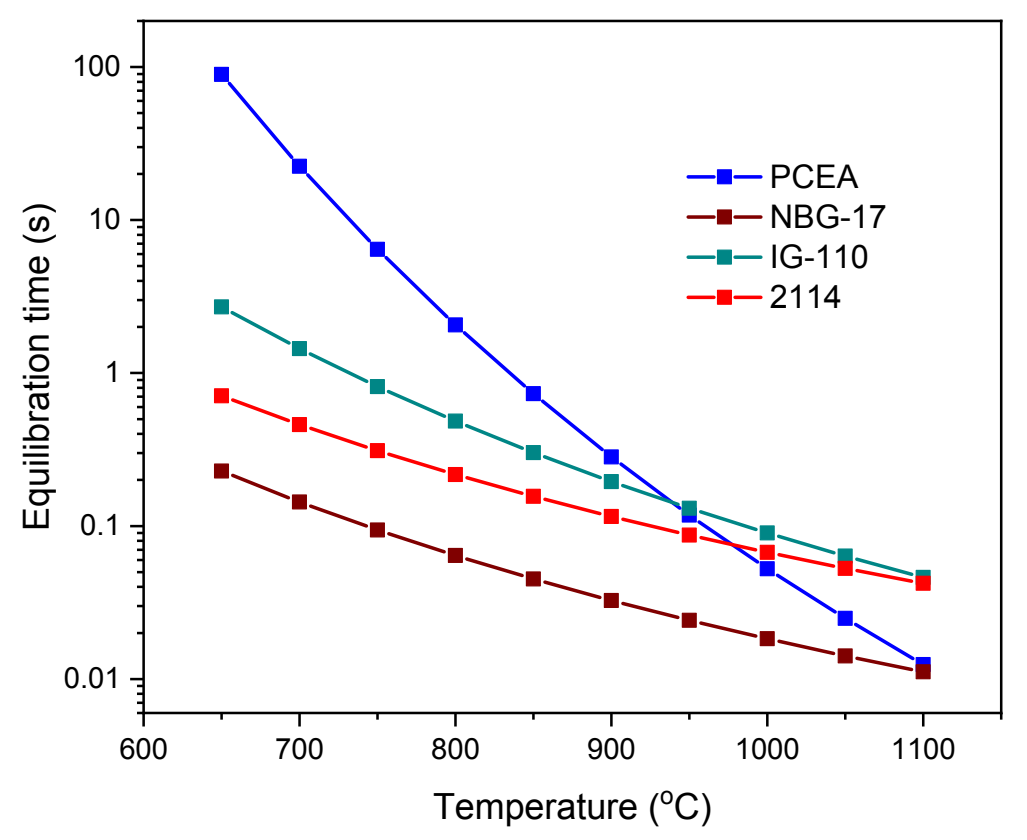

Figure 14. Effect of temperature on the time required for stabilization of a fully developed density profile in the oxidized layer. 


\section{MODELS FOR DENSITY PROFILE IN OXIDIZED GRAPHITE LAYERS}

\subsection{SURFACE OXIDATION RATE}

Comparing diffusivities for $\mathrm{H}_{2} \mathrm{O}$, and $\mathrm{H}_{2}$ in $\mathrm{He}$, Wichner argued that, at temperatures at which mass transfer from the gas phase to the graphite surface is efficient, the surface concentration of the oxidant equals that in the gas phase: $u(0)=U$. This situation is typical for low and medium temperatures. At higher temperatures, one should consider the limitations of mass transfer:

$$
u(0)=U h_{m} /\left(B D_{e f f}\right)
$$

The mass transfer coefficients $h_{m}$, can be estimated from

$$
h_{m}=4 D_{\text {gas }} / d_{\text {hyd }},
$$

where $d_{h y d}$ is the hydraulic diameter of the test section of the laboratory equipment used for experiments and $D_{\text {gas }}$ is the oxidant diffusivity in the free stream gas mixture. The factor 4 is a rough average of the Sherman numbers for several representative situations of reactive gas flow in a tube. The temperature dependence of $D_{\text {gas }}$ causes similar variations for $D_{\text {eff }}$ and for $h_{m}$.

The oxidation rate $R_{s}$ observed at surface $(x=0)$ in mol $\mathrm{C}_{\text {oxidized }} \mathrm{m}^{-2} \mathrm{~s}^{-1}$ may be obtained by integrating the local rate from Eq. (22)

$$
R_{\text {surface }}=\int_{0}^{\infty} R_{L H-l i n e a r}(x) \rho \mathrm{d} x
$$

Using the oxidant distribution from Eq. (9),

$$
u(x)=u(0) \exp (-B x),
$$

where $U=u(0)$ yields after integration and rearrangements:

$$
R_{\text {surface }}=\frac{A u(0)}{B N_{u}}=\sqrt{A D_{\text {eff }} \frac{u(0)}{N_{u}}} .
$$

This result shows that increasing $D_{\text {eff }}$ increases the surface oxidation rate, which is expected since the penetration depth depends on $D_{\text {eff }}$. A higher penetration depth exposes more carbon to oxidation, leading to a higher surface rate.

\subsection{GRAPHITE DENSITY PROFILE}

In the quasi-steady state approximation used in this model, the fully developed oxidant concentration profile retains a nearly stable shape and moves inward. The density profile is the inverse of oxidant concentration profile:

$$
\rho(x)=\rho_{\infty}-\left(\rho_{\infty}-\rho_{0}\right) \frac{u(x)}{u(0)},
$$

where $\rho_{0}$ is the density of an "ultimately" oxidized remnant (perhaps 10-20\% of the original density) and $\rho_{\infty}$ is the density of unoxidized graphite at infinite distance from the exposed surface. When $x$ is large enough, $u(x) \rightarrow 0$ and $\rho(x) \rightarrow \rho_{\infty}$. Conversely, at $x=0, u(x)=u(0)$ and $\rho(x)=\rho_{0}$. Recall that the concentration profile has an exponential variation, as in Eq. (10). Thus, the graphite density profile should 
vary as $(1-\exp (-B x))$ from a minimum value $\rho_{0}$ at the surface and approaching asymptotically the maximum value $\rho_{\infty}$ at some distance in the interior.

This is an important conclusion. It shows that experimentally measured density profiles in the oxidized layer can be compared against oxidant concentration profiles predicted by the model at given kinetic and transport conditions. Both profiles should be described by closely related rates of exponential variation $(B$ for oxidant concentration and $b$ for graphite density). We use this conclusion later in the document.

\subsection{OXIDATION BY MOISTURE AND HYDROGEN BUILDUP}

\subsubsection{Coupled Transport Equations for Water and Hydrogen}

According to both the LH and BLH models, the rate of graphite oxidation by moisture is slowed down in the presence of hydrogen. As a reaction product of oxidation by water, hydrogen formed in the graphite porosity acts as a reaction inhibitor through mechanisms still not yet elucidated. This is a great complication for the analyst who tries to develop a comprehensive model. Wichner ${ }^{5,6}$ used two coupled transport equations for $\mathrm{H}_{2} \mathrm{O}$ and $\mathrm{H}_{2}$;

$$
\begin{gathered}
0=D_{u} \nabla^{2} u-\text { Rate }_{I} \rho N_{\mathrm{H} 20} \\
0=D_{v} \nabla^{2} v+\text { Rate }_{I} \rho N_{\mathrm{H} 2}
\end{gathered}
$$

\begin{tabular}{|c|c|c|}
\hline where & $D_{u}, D_{v}$ & effective diffusivities for $\mathrm{H}_{2} \mathrm{O}$ and $\mathrm{H}_{2}$ \\
\hline & $u$ & $\mathrm{H}_{2} \mathrm{O}$ concentration (moles $\mathrm{H}_{2} \mathrm{O} / \mathrm{m}^{3}$-void) \\
\hline & $v$ & $\mathrm{H}_{2}$ concentration (moles $\mathrm{H}_{2} / \mathrm{m}^{3}$-void) \\
\hline & $N_{H 2 O}$ & stoichiometric number for $\mathrm{H}_{2} \mathrm{O}$ (moles $\mathrm{H}_{2} \mathrm{O}$ consumed per mole $\mathrm{C}$ oxidized) \\
\hline & $N_{H 2}$ & stoichiometric number for $\mathrm{H}_{2}$ (moles $\mathrm{H}_{2}$ produced per mole $\mathrm{C}$ oxidized) \\
\hline & Rate $_{1}$ & reaction rate in diffusion-free conditions (also called zone I) \\
\hline
\end{tabular}

Equations (33) and (34) are coupled because $u$ and $v$ are both contained in Rate ${ }_{I}$. The rate of oxidation determines the rate of production of $\mathrm{H}_{2}$, which in turn has an inhibition effect on the rate of oxidation. Linearizing Rate ${ }_{I}$ allows sequential solutions for $u$ and $v$ but may not portray the true inhibiting effect of $\mathrm{H}_{2}$.

\subsubsection{Effect of Linearization}

Wichner used the linearized LH rate, Rate $_{L H-l i n}$ as the rate for diffusion-free conditions, Rate ${ }_{I}$, in Eq. (3). With the rates based on fully characterized LH and BLH models from Eqs. (11) and (12), we compared the variation of experimental data $P_{\mathrm{H} 2 \mathrm{O}}$ against the predicted rates based on linearized LH and full $\mathrm{LH}$ and BLH models. The comparison was made for all four graphite grades at three temperatures in the range selected for analysis of oxidized layer profiles (Figure 15). The purpose was to compare the errors introduced by linearization versus the predictions of the full models. The plots show that the linearized equation follows satisfactorily the trend of the experimental data. The agreement is better at higher temperatures $\left(900-1000^{\circ} \mathrm{C}\right)$ and $P_{\mathrm{H} 2 \mathrm{O}}$ in the range of 200-300 Pa. These are the conditions used for preparation of specimens with larger weight loss employed for analysis of oxidized layer profiles (Chapter 8). 


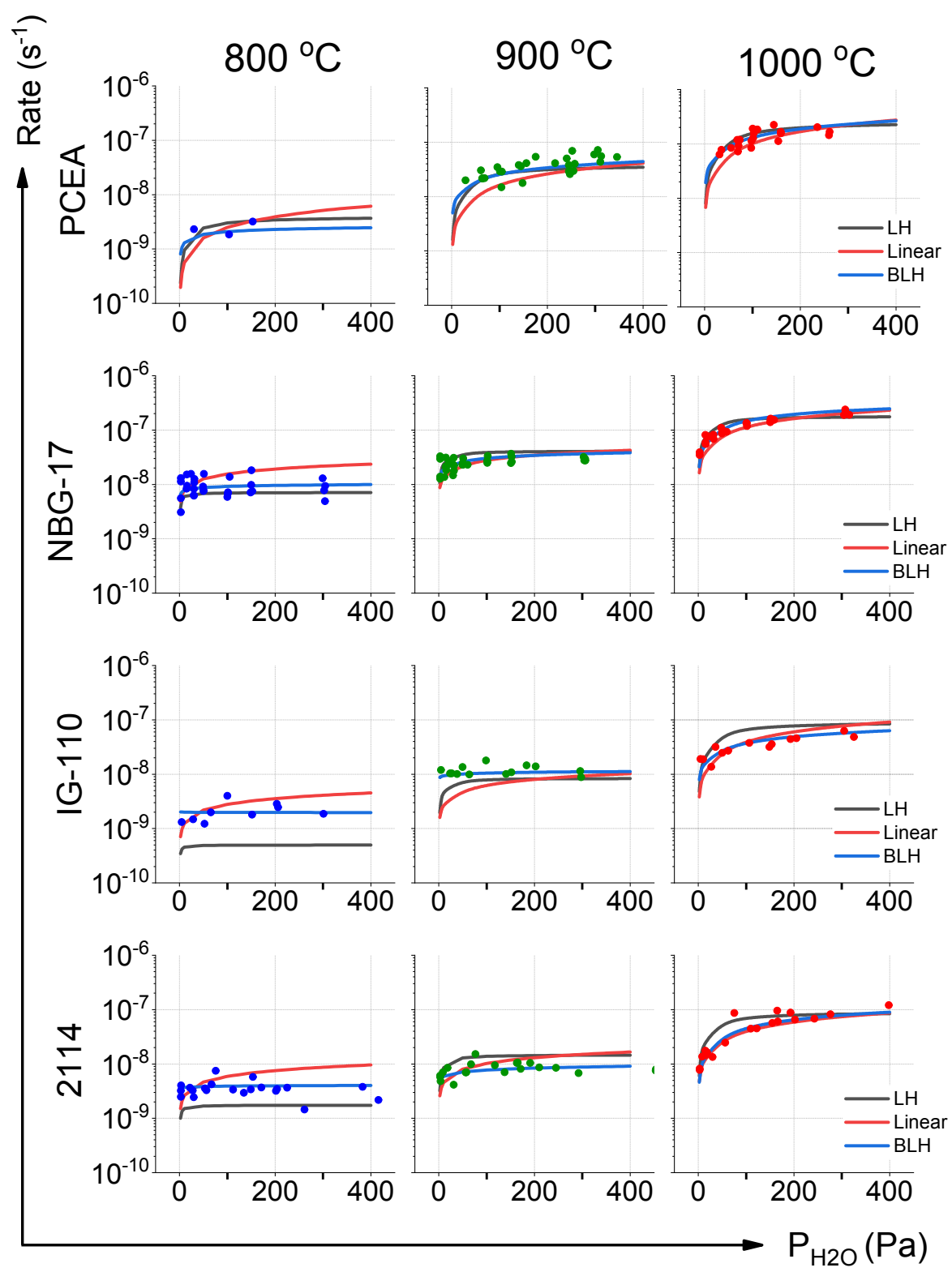

Figure 15. Comparison of oxidation rate variation with $P_{\mathrm{H} 2 \mathrm{O}}$ obtained from fitting with experimental results data for all graphite grades at 800,900 and $1000{ }^{\circ} \mathrm{C}$. Fitting by LH and BLH models is compared with fitting using the linearized rate equation of the $\mathrm{LH}$ model.

\subsubsection{Estimation of Water and Hydrogen Concentration Profiles in a Finite Slab}

The decoupled transport equations of $\mathrm{H}_{2} \mathrm{O}$ and $\mathrm{H}_{2}$ at quasi-steady state become

$$
\begin{aligned}
& 0=D_{u} u "-A_{u} u . \\
& 0=D_{v} v^{\prime \prime}+A_{v} v .
\end{aligned}
$$

The parameters $A_{u}$ and $A_{v}$ have similar definitions as in Eq. (6) but with their respective stoichiometric numbers: 


$$
\begin{aligned}
& A_{u}=k_{u} \exp \left(-\frac{E_{u}}{\mathrm{RT}}\right) \rho N_{u} \mathrm{R} T \\
& A_{v}=k_{u} \exp \left(-\frac{E_{u}}{\mathrm{RT}}\right) \rho N_{v} \mathrm{R} T
\end{aligned}
$$

In a more convenient form,

$$
\begin{aligned}
& 0=u^{\prime \prime}-B_{u}{ }^{2} u, \\
& 0=v^{\prime \prime}+B_{v}{ }^{2} u,
\end{aligned}
$$

where $B_{u}{ }^{2}=A_{u} / D_{u}$ and $B_{v}{ }^{2}=A_{v} / D_{v}$. The density profile of $\mathrm{H}_{2} \mathrm{O}$ in a thick slab of half-width $L$ is obtained by solving Eq. (35a) with the appropriate boundary conditions:

$$
u(x)=u(0)\left[\cosh \left(B_{u} x\right)-\tanh \left(B_{u} L\right) \sinh \left(B_{u} x\right)\right] .
$$

The $\mathrm{H}_{2}$ concentration profile is obtained by substituting Eq. (36) into Eq. (35b) and integrating twice:

$$
v(x)=v(0)+u(0)\left(B_{v}{ }^{2} / B_{u}{ }^{2}\right)\left[1-\cosh \left(B_{u} x\right)+\tanh \left(B_{u} L\right) \sinh \left(B_{u} L\right)\right]
$$

where $u(0)$ and $v(0)$ are the concentrations of $\mathrm{H}_{2} \mathrm{O}$ and $\mathrm{H}_{2}$ in graphite at the surface $(x=0)$, which are equal to $U$ and $V$, the gas phase concentrations of $\mathrm{H}_{2} \mathrm{O}$ and $\mathrm{H}_{2}$ at the surface. For convection in laminar regime Wichner obtained the surface concentrations as

$$
\begin{gathered}
u(0)=\frac{h_{u} U}{h_{u}+D_{u} B_{u} \tanh \left(B_{u} L\right)}, \\
v(0)=V+\left[\frac{u(0) D_{v}}{h_{v}}\right]\left(\frac{B_{v}{ }^{2}}{B_{u}{ }^{2}}\right) \tanh \left(B_{u} L\right),
\end{gathered}
$$

where $h_{u}$ and $h_{v}$ are mass transfer coefficients for $\mathrm{H}_{2} \mathrm{O}$ and $\mathrm{H}_{2}$. Thus, the concentration profiles for $\mathrm{H}_{2} \mathrm{O}$ and $\mathrm{H}_{2}$ in a finite slab are given by Eqs. (36) and (37), with surface concentrations given by Eqs. (38) and (39). These equations can be used for sequential estimation, which neglects the interrelation between $\mathrm{H}_{2} \mathrm{O}$ and $\mathrm{H}_{2}$ consumption, generation, and transport. This sequential estimation method may overestimte both $\mathrm{H}_{2} \mathrm{O}$ and $\mathrm{H}_{2}$ concentrations: $\mathrm{H}_{2} \mathrm{O}$ because the $\mathrm{H}_{2}$ inhibition is not accounted for, and $\mathrm{H}_{2}$ because its source, which is the rate of oxidation by $\mathrm{H}_{2} \mathrm{O}$, is overestimated. Based on Wichner's analysis, the sequential procedure should be fairly accurate when the $\mathrm{H}_{2}$ inhibition effect is small. This is expected for high water partial pressures, for example when $P_{H 2 O}>300 \mathrm{~Pa}^{5}{ }^{5}$ With these caveats, the procedure developed by Wichner provides a fairly good account of the distributions of $\mathrm{H}_{2} \mathrm{O}$ and $\mathrm{H}_{2}$ in graphite. More details and a few illustrative plots are available in the original report. ${ }^{5}$

\subsubsection{Water Transport in a Long Cylinder}

The oxidation experiments performed in the laboratory used cylindrical specimens, not flat slabs. That does not change the analysis but requires the use of cylindrical coordinates. With the new coordinates, and assuming linearized kinetics derived from the $\mathrm{LH}$ model, the quasi-steady $\mathrm{H}_{2} \mathrm{O}$ transport equation in a long cylinder can written as

$$
0=D_{u} \frac{1}{r} \frac{\mathrm{d}}{\mathrm{d} r}\left(r \frac{\mathrm{d} u}{\mathrm{~d} r}\right)-A_{u} u
$$


or in a more convenient form,

$$
0=\frac{1}{r} \frac{\mathrm{d}}{\mathrm{d} r}\left(r \frac{\mathrm{d} u}{\mathrm{~d} r}\right)-B_{u}{ }^{2} u
$$

This equation was discussed by Wichner. ${ }^{5}$ Using boundary conditions specific to the cylindrical geometry, the solution is

$$
u(r)=u(a) \mathrm{I}_{0}\left(B_{u} r\right) / \mathrm{I}_{0}\left(B_{u} a\right) .
$$

The cylinder's center is at $r=0$ and its surface is at $r=a$. The surface concentration $u(a)$ is determined by the free stream concentration of $\mathrm{H}_{2} \mathrm{O}$, the surface mass transfer coefficient, and the rate of diffusion away from the surface:

$$
u(a)=\frac{h_{u} U}{h_{u}+D_{u} B_{u}\left[\frac{1_{1}\left(B_{u} a\right)}{I_{0}\left(B_{u}\right)}\right]} .
$$

In the previous equations, $\mathrm{I}_{0}$ and $\mathrm{I}_{1}$ are modified Bessel functions of the first and second kind, respectively. They are available for calculations in Excel spreadsheets. Thus, the $\mathrm{H}_{2} \mathrm{O}$ concentration in the cylinder is given by Eq. (43) and the surface concentration is given by Eq. (44).

\subsubsection{Hydrogen Concentration in a Long Cylinder}

Similar to Eq. (42), the quasi-steady state transport equation for $\mathrm{H}_{2}$ in a long cylinder is

$$
0=D_{v_{r}} \frac{1}{\mathrm{~d} r}\left(r \frac{\mathrm{d} v}{\mathrm{~d} r}\right)+A_{v} u
$$

which reduces to the more convenient form

$$
0=\frac{1}{r} \frac{\mathrm{d}}{\mathrm{d} r}\left(r \frac{\mathrm{d} v}{\mathrm{~d} r}\right)+B_{v}^{2} u
$$

where $v \quad$ local concentration of $\mathrm{H}_{2}$ in voids ( $\mathrm{mol} / \mathrm{m}^{3}$-void)

$D_{v} \quad$ effective diffusivity of $\mathrm{H}_{2}$

$A_{v} \quad$ rate of $\mathrm{H}_{2}$ generation, which equals the rate of $\mathrm{H}_{2} \mathrm{O}$ consumption (based on the selected model, either linearized LH or full LH and BLH) but with its own stoichiometric

$B_{v}{ }^{2} \quad A_{v} / D_{v}$

$N_{\mathrm{v}} \quad$ stoichiometric constant, $\mathrm{mol} \mathrm{H}_{2}$ produced/mol C oxidized

Recasting Eq. (36b) and using the results for $\mathrm{H}_{2} \mathrm{O}$ distribution yields

$$
\frac{1}{r} \frac{\mathrm{d}}{\mathrm{d} r}\left(r \frac{\mathrm{d} v}{\mathrm{~d} r}\right)=-B_{v}{ }^{2} u(a) \mathrm{I}_{0}\left(B_{u} r\right) / \mathrm{I}_{0}\left(B_{u} a\right) .
$$

After two integrations, the concentration distribution of $\mathrm{H}_{2}$ is obtained as

$$
v(r)=v(a)+\left(B_{v} / B_{u}\right)^{2} \cdot u(r)\left[1-\frac{\mathrm{I}_{0}\left(B_{u} r\right)}{\mathrm{I}_{0}\left(B_{u} a\right)}\right] .
$$


The concentration of $\mathrm{H}_{2}$ at the graphite surface, $v(a)$, is obtained from the free stream concentration, $V$, and the mass transfer coefficient for $\mathrm{H}_{2}, h_{v}$. The direction of $\mathrm{H}_{2}$ mass transfer is always from graphite to free stream:

$$
h_{v}(v(0)-V)=-D_{v}(\mathrm{~d} v / \mathrm{d} r) \text { at } r=a .
$$

Since the mass transfer coefficient in the laminar regime is proportional to the diffusivity, $h_{v}>>h_{u}$. Evaluating the derivative and solving for $v(a)$ yields

$$
v(a)=V+\left(\frac{D_{v}}{h_{v}}\right)\left(\frac{B_{v}{ }^{2}}{B_{u}}\right) u(a) \frac{\mathrm{I}_{1}\left(B_{v} a\right)}{\mathrm{I}_{0}\left(B_{v} a\right)} .
$$

Thus, the $\mathrm{H}_{2}$ concentration in the graphite cylinder is given by Eq. (47) and the surface concentration is given by Eq. (49).

\subsubsection{Surface Oxidation Rate by Moisture of a Long Cylinder}

The surface oxidation rate, $R_{\text {surface }}\left(\right.$ moles $\mathrm{C}_{\text {oxidized }} \mathrm{m}^{-1} \mathrm{~s}^{-1}$ ), is obtained by integrating from $r=0$ (center) to $r=a($ surface):

$$
R_{\text {surface }}=\int_{0}^{a} \text { Rate }_{I}(r) r 2 \pi r \mathrm{~d} r
$$

where Rate $_{\mathrm{I}}$ is the diffusion-free rate (zone I) in the selected kinetic model. For linearized LH kinetics, Rate $_{\mathrm{I}}$ is

$$
R_{L H-l i n}(r)=k_{u} \exp \left(-\frac{E_{u}}{\mathrm{R} T}\right) \mathrm{R} T u(r)
$$

Substituting Eq. (51) into Eq. (50), and using Eq. (44) for $u(a)$ and Eq. (43) for $u(r)$, yields for the surface rate

$$
R_{\text {surface }}=2 \pi k_{u} \exp \left(-\frac{E_{u}}{\mathrm{RT}}\right) \rho \mathrm{R} T \frac{a u(a)}{B_{u}}\left[\frac{I_{1}\left(B_{u} a\right)}{I_{0}\left(B_{u} a\right)}\right]
$$

\subsubsection{Efficiency of Oxidation by Moisture of a Long Graphite Cylinder}

Oxidation efficiency, $\eta$, is defined as the ratio between the diffusion-limited oxidation rate, Rate ${ }_{I}$, and an assumed uniform oxidation rate:

$$
R_{\text {surface }}(\text { uniform })=\text { Rate }_{I}(a) \rho \pi a^{2} .
$$

The latter is evaluated using the $\mathrm{H}_{2} \mathrm{O}$ concentration in the graphite as constant, at the surface value, $u(a)$. The efficiency of oxidation of a graphite cylinder by water is therefore

$$
\eta=\frac{2}{a B_{u}}\left[\frac{\mathrm{I}_{1}\left(B_{u} a\right)}{\mathrm{I}_{0}\left(B_{u} a\right)}\right] .
$$

This ratio, which is another form of the Thiele modulus used in catalysis, shows what the conditions are where oxidation is expected to be (more or less) uniform $(\eta>0.8)$ or localized at the exposed surface $(\eta<0.8)$. With the increase in temperature, and keeping other conditions constant, $\eta$ decreases. With an increase in the reaction rate faster than the diffusivity rate, oxidation transitions from conditions typical of 
a chemical kinetics-controlled mechanism (zone I, $\eta>0.8$ ) to conditions typical of a diffusion-controlled mechanism (zone II, $\eta<0.8$ ). 


\section{MEASUREMENTS ON DENSITY PROFILES OF OXIDIZED GRAPHITE SPECIMENS}

\subsection{NEW OXIDATION EXPERIMENTS IN CONTROLLED CONDITIONS}

Kinetic measurements performed with each of the four grade of graphite produced numerous specimens with various oxidation histories and final weight loss levels typically less than $1 \%$. These levels are too low for analyzing the oxidized layer and estimating the oxidant penetration depth and graphite density profiles. To accomplish these goals, new sets of oxidized specimens had to be produced for each graphite grade in controlled oxidation conditions. The final weight loss target was set at $5 \%$, assuming that at that level, the effect of oxidation would become visible for optical microscopy observations. Indeed, we have previously shown that the oxidized layer after oxidation in air at $5 \%$ weight loss was easily visualized and analyzed by optical microscopy. ${ }^{8,9}$

\subsubsection{Preparation of Specimens Oxidized at Higher Weight Loss}

Cylindrical specimens for high-weight- loss oxidation were machined from the same graphite sub-block as the ones used for kinetic measurements. At the time of the experiments with PCEA graphite, it was still not clear how deeply the oxidized layer formed from reactions with moisture would penetrate below the surface. Therefore, PCEA specimens for oxidation observations were machined with larger diameters $(8 \mathrm{~mm})$ with the intention to provide enough room for development and observation of the oxidized layer. Indeed, the oxidized layers were easily observed on PCEA samples. The remaining graphites for penetration depth examination were machined like their counterparts for kinetic studies (4 mm for NBG17 and IG-110 and $5 \mathrm{~mm}$ for 2114). With the exception of superfine grade IG-110, that was a good choice, but the IG-110 specimens may have had diameters too small for any observation of surface contrast in the oxidized layer; they may have been totally penetrated by oxidation even at high temperatures.

The specially prepared specimens were oxidized in the system previously used for kinetic studies. Each sample was obtained at a constant temperature, at constant gas flow rate and water vapor pressure (no hydrogen added). See the information provided in Table 9. These preparations took variable lengths of time, as the oxidation reaction varied considerably with temperature. The first attempt to prepare a PCEA sample oxidized at $800{ }^{\circ} \mathrm{C}$ was abandoned because, with the extremely slow oxidation rate at this temperature, it would have taken many weeks to reach a substantial weight loss. At higher temperatures, oxidation was faster, but it still took 9 days at $900{ }^{\circ} \mathrm{C}$ to reach only a $2.6 \%$ weight loss.

There were also unexpected interruptions of long oxidation runs, caused by the need to replace gas tanks, electrical failures, computer failures, and so on. In all such unpredicted breaks, the specimens were kept in the TAG instrument under He and the process continued as soon as was possible.

Oxidized specimens were measured and weighed to determine the final weight loss. Then the specimens were cut transversally, and one half was mounted in epoxy resin and polished for examination by optical microscopy. 
Table 9. Specimens oxidized at higher weight loss for observation of oxidized layer.

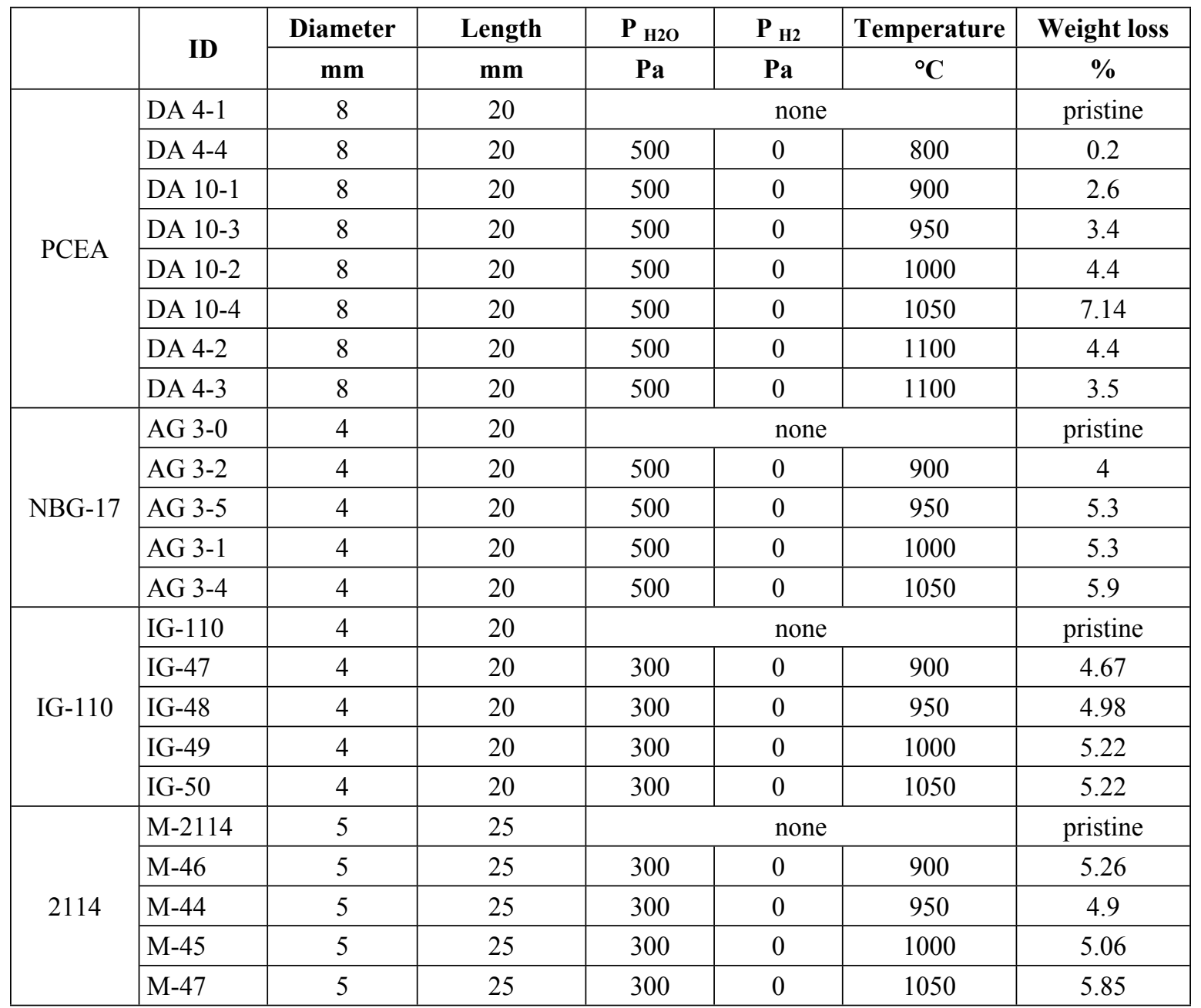

\subsection{EXPERIMENTAL OBSERVATION OF OXIDIZED LAYERS}

\subsubsection{Optical Microscopy with Automated Data Analysis}

Examination by optical microscopy followed a protocol developed initially for analysis of graphite specimens oxidized in air. ${ }^{57}$ All oxidized specimens were cut transversally and mounted in plastic fixtures using epoxy resin. Mounted specimens were engraved for identification and polished for metallographic examination using a sequence of $\mathrm{SiC}$ grinding papers with gradually finer grits, followed by polishing cloths, and terminated by final vibratory polishing in a colloidal silica/water mixture.

A Keyence microscope equipped with a motorized stage and autofocus control was used to collect stitched images of the specimens' sectioned surfaces. Several magnifications were used: $1000 \times$ for detailed examination, $500 \times$ for larger details, and $200 \times$ to obtain a general image of the transversal section. All images were collected in normal and polarized light. Spatial calibration of the microscope was performed with special grids.

Analysis of the porosity development was performed on mosaic images collected at $200 \times$. Usually, the area of interest was a rectangle with width of 1.0-1.5 $\mathrm{mm}$ and length slightly larger than the specimen's 
diameter. Mosaic images were analyzed by automated image analysis (Image Pro) following the procedure developed previously. ${ }^{57}$ Images were converted to an 8-bit grayscale format, and the contrast was enhanced using the linear equalization function. A histogram analysis provided the frequency of pixels as a function of respective gray shade intensities, between 0 (extreme black) and 255 (extreme white). Polished graphite surfaces reflect light and appear with luminous intensities (large pixel values). Pores are less reflective and appear as regions of lower pixel values. Figure 16 shows examples of pixel intensity histograms associated with images of graphite NBG-17 (pristine and after oxidation at four temperatures).

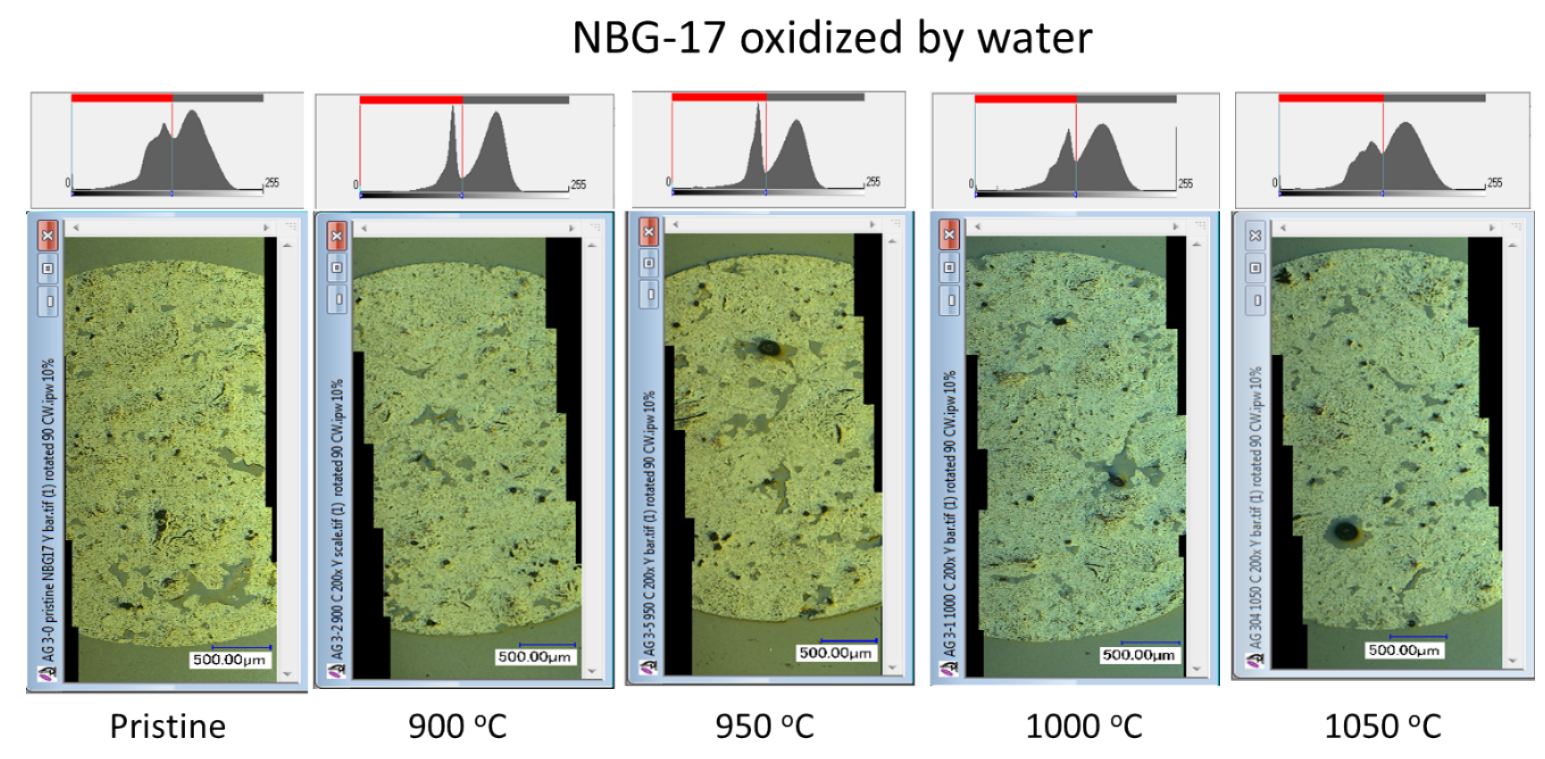

Figure 16. Examples of pixel intensity distributions extracted from images of pristine and oxidized NBG-17 specimens.

A segmentation procedure was used next to select only the gray tones associated with pores. They are marked by the red segments in the histograms shown on top of Figure 16. The selected objects were then counted and classified by size. A somehow arbitrary threshold was set at $100 \mu \mathrm{m}^{2}$ to separate fine pores caused by oxidation from all other, larger pores. A two-color code was used to differentiate between fine pores (red) and larger pores (green). This procedure showed gradual development of fine pores near the surface of larger grain graphites and their segregation in large numbers with the increase in oxidation temperature. This is very clear for PCEA graphite (Figure 17) and to some extent for NBG-17 (Figure 18 ), both medium-fine grain grades. In contrast, it is very difficult to distinguish new pores formed by oxidation in superfine grain graphites IG-110 (Figure 19) and 2114 (Figure 20). 


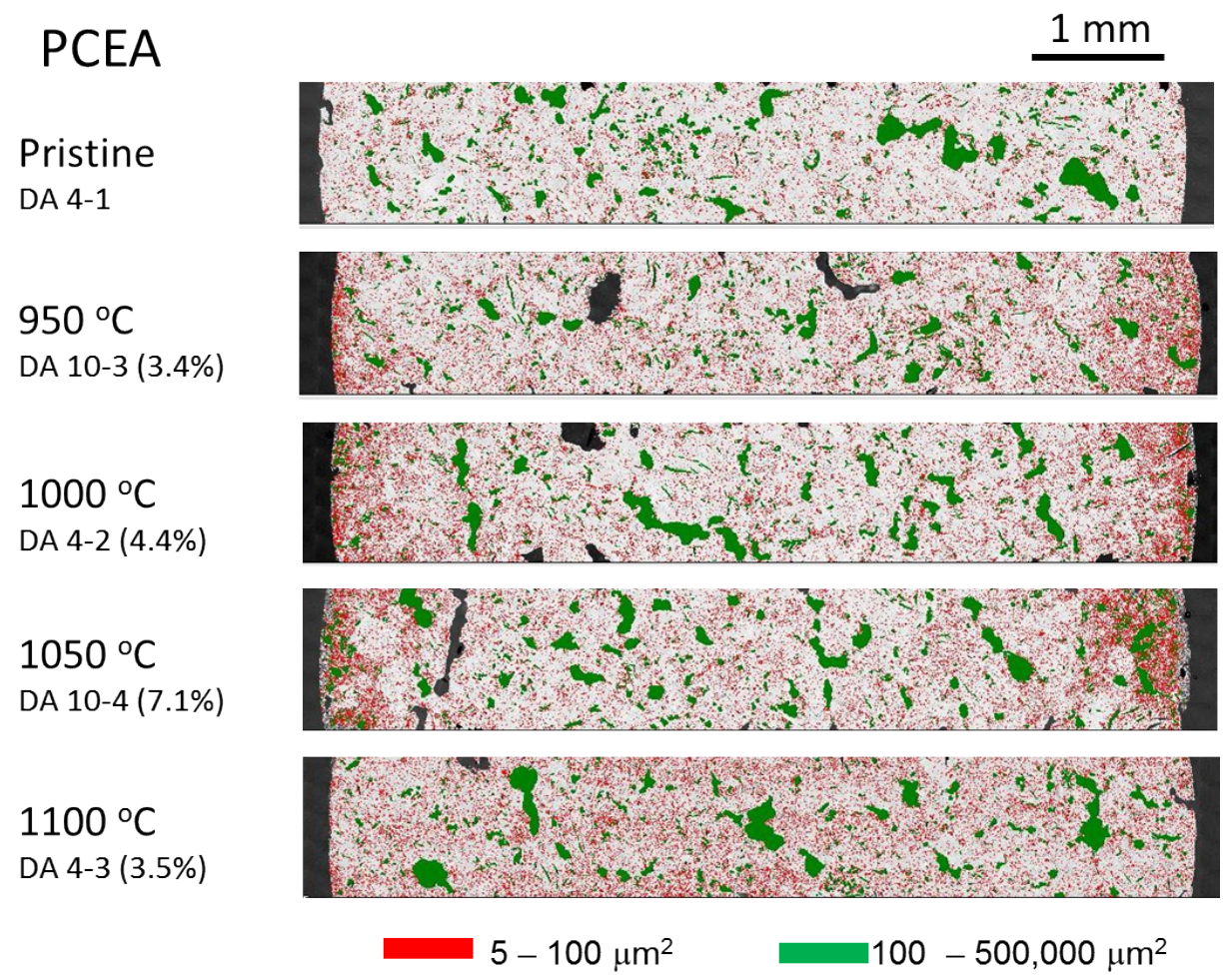

Figure 17. Color-coded visualization of fine pores in the oxidized layer after oxidation by water of PCEA graphite (6 mm diameter).

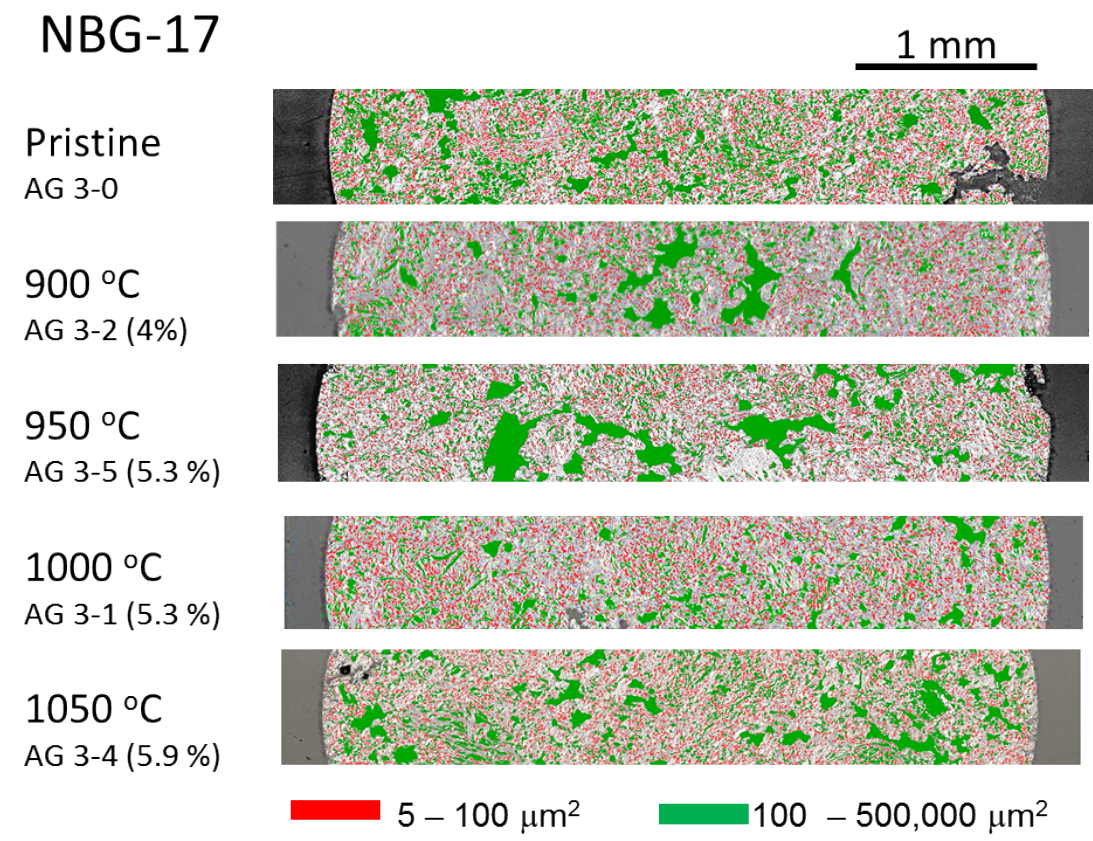

Figure 18. Color-coded visualization of fine pores in the oxidized layer after oxidation by water of NBG-17 graphite (4 mm diameter). 

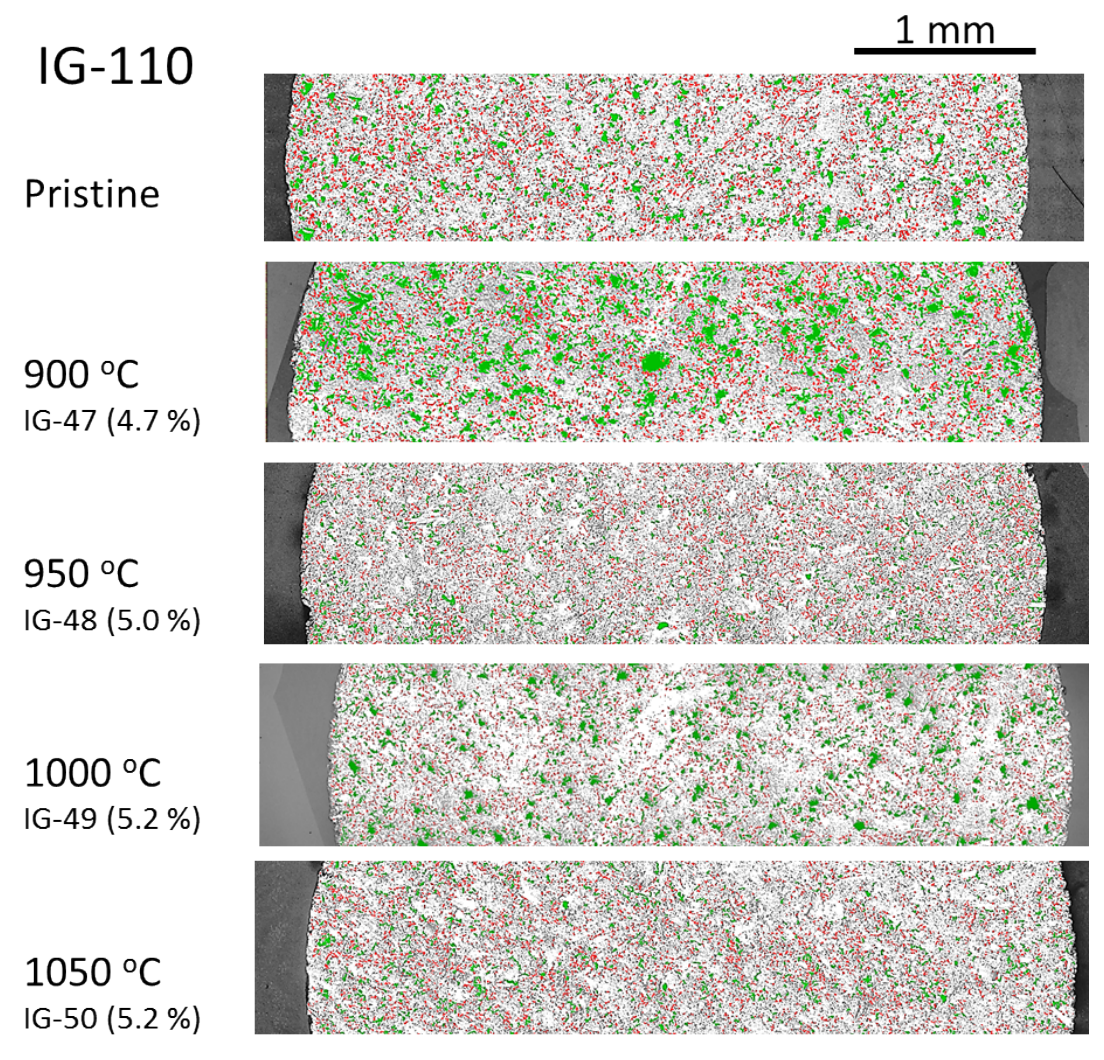

$5-100 \mu \mathrm{m}^{2} \quad 100-500,000 \mu \mathrm{m}^{2}$

Figure 19. Color-coded visualization of fine pores in the oxidized layer after oxidation by water of IG-110 graphite ( $4 \mathrm{~mm}$ diameter). 

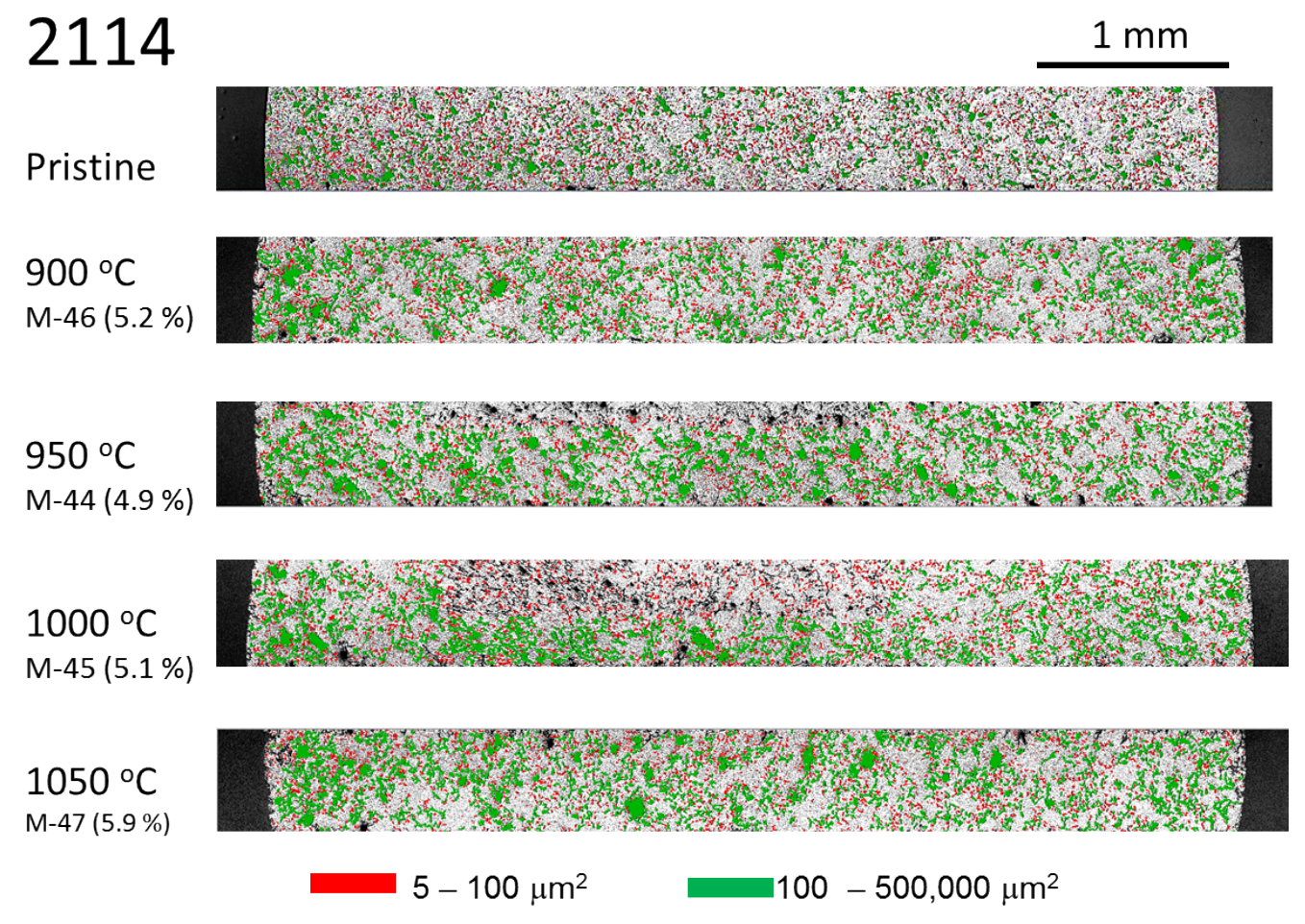

Figure 20. Color-coded visualization of fine pores in the oxidized layer after oxidation by water of 2114 graphite ( $5 \mathrm{~mm}$ diameter).

Next, the line profile of pixel intensities versus the distance from the edge was measured for each image, in its respective rectangular area of interest. The results were very different between medium-fine grades and superfine grades. Graphites with larger grains, PCEA and NBG-18, contain large pores that distort pixel intensity profiles and contribute "dark" areas (low pixel values) that interfere with the images of fine pores resulting from oxidation. It was necessary to filter (smooth) the raw pixel profiles (as was done for PCEA) or to combine profiles from diametrically opposed positions at the two edges of the area of interest and then analyze the properly overlapped data (as was done for NBG-17). The "noise" caused by large random pores was not present in superfine grades IG-110 and 2114.

This analysis provided interesting results. For PCEA. it was clear that the groups of fine pores near the surfaces of specimens oxidized at high temperature (Figure 17) form rounded-down distributions of pixel intensities toward the edge. Similar effects, although less evident, were found for NBG-17. These profiles were fitted with the exponential decay function

$$
y=y_{0}+a \exp \left(-\frac{1}{\tau} x\right)
$$

where $y$ (no units) is the pixel intensity, $x(\mu \mathrm{m})$ is the distance from the surface, and $\tau(\mu \mathrm{m})$ is a characteristic dimension that shows the rate of decay. Figure 21 shows the averaged pixel intensity distributions near the surfaces (1-2 mm) of pristine and oxidized graphites and their fitting with Eq. (55). Table 10 shows the fitted parameters in Eq. (53) describing the exponential decay of the oxidized layer density profiles. 


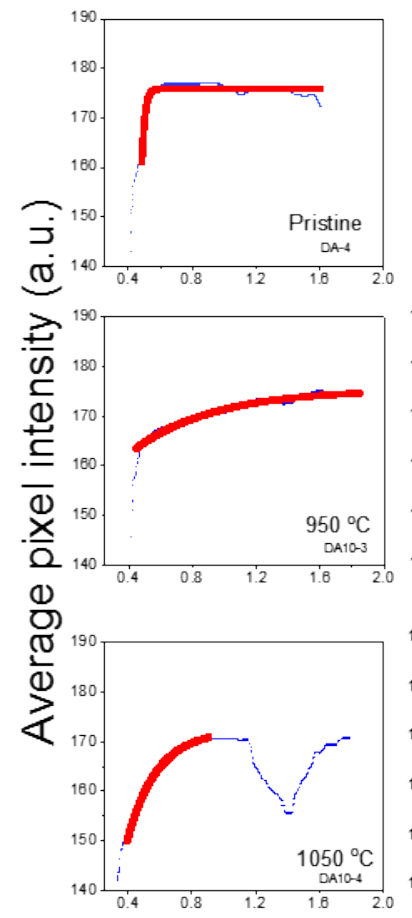

PCEA

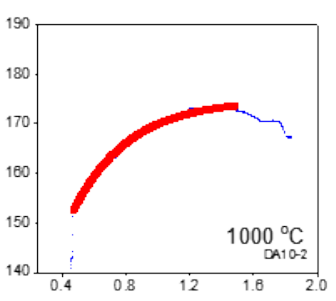

Radial distance $(\mathrm{mm})$

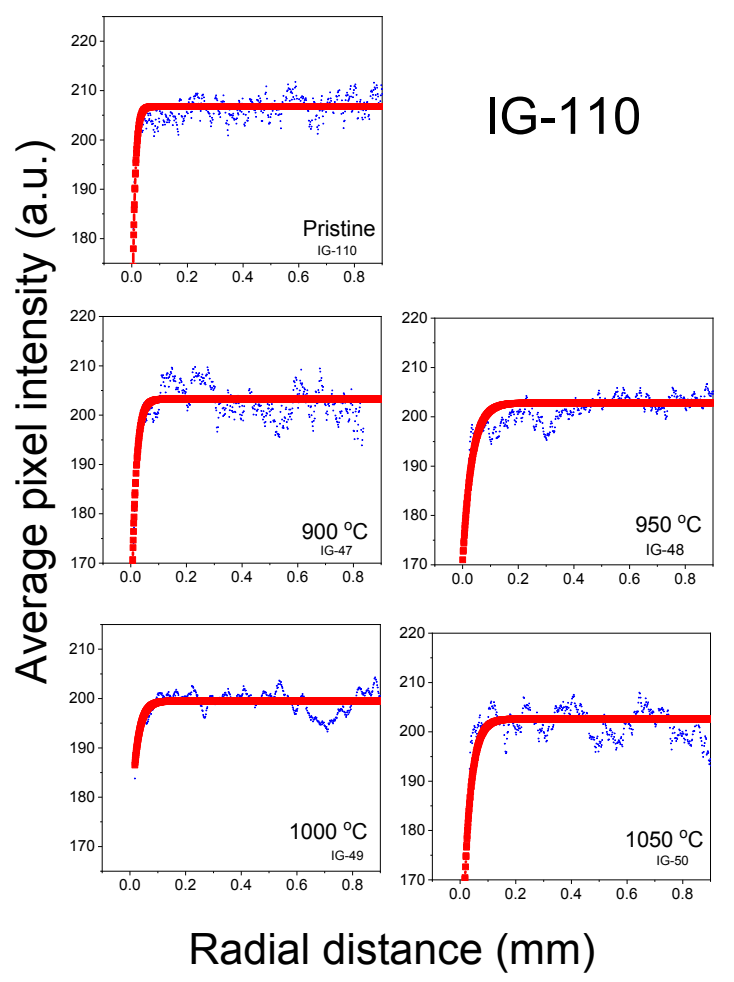

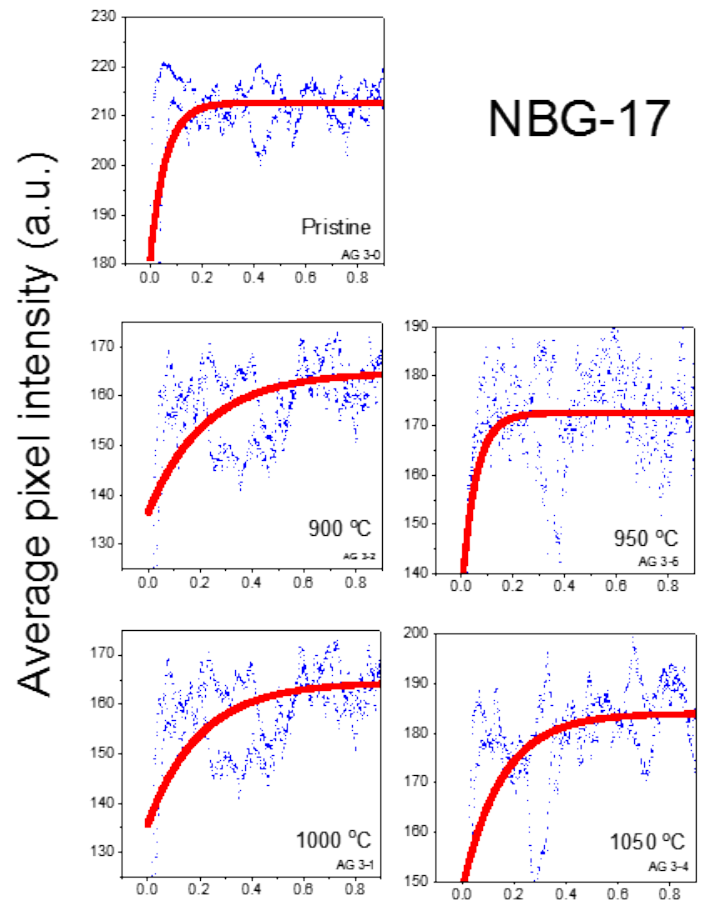

Radial distance $(\mathrm{mm})$

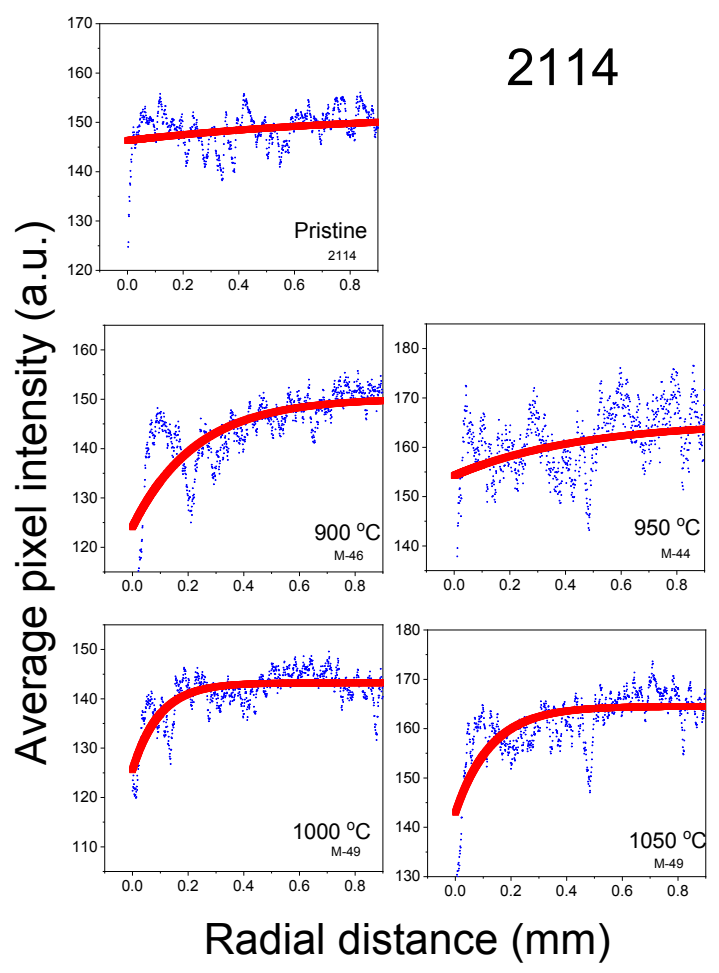

Figure 21. Density profiles of oxidized layers fitted with exponential decay function. 
Table 10. Fitted parameters of exponential decay functions associated with oxidized layer profiles.

\begin{tabular}{|c|c|c|c|c|c|c|c|c|c|}
\hline & & \multicolumn{4}{|c|}{ Microscopy } & \multicolumn{4}{|c|}{ Tomography } \\
\hline & & $\mathrm{y}_{0}$ & $\mathbf{a}$ & $\tau(\mu \mathbf{m})$ & \pm error $\tau(\mu \mathbf{m})$ & $\mathbf{y}_{0}$ & $\mathbf{a}$ & $\tau(\mu \mathrm{m})$ & $\pm \operatorname{error} \tau(\mu \mathrm{m})$ \\
\hline \multirow{6}{*}{ PCEA } & Pristine & 152 & -5.8 & 877 & 122 & & & & \\
\hline & $900^{\circ} \mathrm{C}$ & 150 & -26 & 507 & 17 & & & & \\
\hline & $950^{\circ} \mathrm{C}$ & 166 & -11 & 350 & 24 & 1 & 0 & 543 & 23 \\
\hline & $1000^{\circ} \mathrm{C}$ & 148 & -17 & 184 & 10 & 0.99 & 0 & 215 & 21 \\
\hline & $1050^{\circ} \mathrm{C}$ & 164 & -21 & 142 & 3 & 1 & 0 & 310 & 10 \\
\hline & $1100^{\circ} \mathrm{C}$ & & & & & 1 & 0 & 134 & 6 \\
\hline \multirow{5}{*}{ NBG-17 } & Pristine & 213 & -31 & 59.4 & 1.1 & 0.99 & 0.06 & 121 & 160 \\
\hline & $900^{\circ} \mathrm{C}$ & 165 & -28 & 220 & 5 & & & & \\
\hline & $950^{\circ} \mathrm{C}$ & 172 & -38 & 54 & 1.8 & & & & \\
\hline & $1000^{\circ} \mathrm{C}$ & 164 & -29 & 202 & 4.5 & 1.02 & -0.03 & -1754 & 606 \\
\hline & $1050^{\circ} \mathrm{C}$ & 184 & -35 & 153 & 4.6 & 0.98 & 0 & -708 & 99 \\
\hline \multirow{5}{*}{ IG-110 } & Pristine & 207 & -17921 & 49 & 1.9 & & & & \\
\hline & $900^{\circ} \mathrm{C}$ & 203 & -1971 & 105 & 4.8 & & & & \\
\hline & $950^{\circ} \mathrm{C}$ & 204 & -23297 & 51 & 1.3 & & & & \\
\hline & $1000^{\circ} \mathrm{C}$ & 199 & -5009 & 62 & 2 & & & & \\
\hline & $1050^{\circ} \mathrm{C}$ & 202 & -4023 & 81 & 3.3 & & & & \\
\hline \multirow{5}{*}{2114} & Pristine & 152 & -5.8 & 878 & 122 & & & & \\
\hline & $900^{\circ} \mathrm{C}$ & 150 & -26 & 228 & 8.2 & & & & \\
\hline & $950^{\circ} \mathrm{C}$ & 165 & -11 & 452 & 49 & & & & \\
\hline & $1000^{\circ} \mathrm{C}$ & 148 & -18 & 97 & 5.2 & & & & \\
\hline & $1050^{\circ} \mathrm{C}$ & 164 & -22 & 128 & 7.2 & & & & \\
\hline
\end{tabular}

\subsubsection{Tomography}

The second halves of oxidized PCEA and NBG-17, not used for microscopy examination, were analyzed by x-ray tomography (XRT), courtesy of Dr. Josh Kane (INL). After scanning, the images were projected on the cylinder's flat base and the normalized density ratios were radially averaged. Figure 22 shows good agreement between overlapped color-coded optical microscopy images of the oxidized layer and the tomography images, revealing lower-density zones near the edges. Both techniques show that the higher the oxidation temperature, the narrower is the oxidized layer near the surface. 


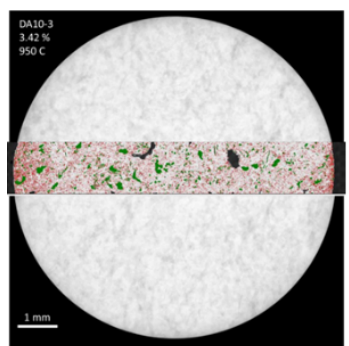

$950 \mathrm{C}$

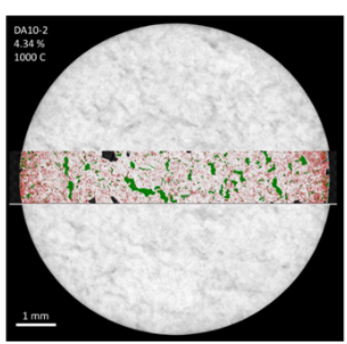

$1000 \mathrm{C}$

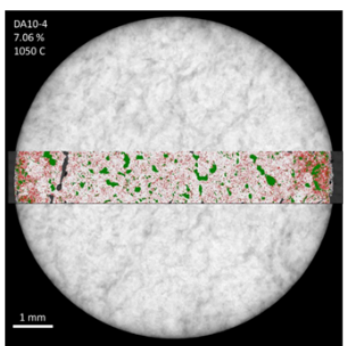

$1050 \mathrm{C}$

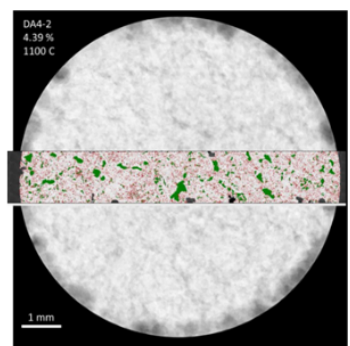

$1100 \mathrm{C}$

Figure 22. Comparison between tomography and microscopy results for oxidized PCEA specimens (6 mm diameter).

Quantitative analysis of density-normalized and radially averaged tomography data used the same exponential decay function, Eq. (53). Parameters are provided for comparison in Table 10. Figure 23 illustrates the complementarity of results obtained from optical microscopy and tomography for the sample of PCEA graphite. The results represent density profiles in two halves of the same four specimens oxidized at different temperatures.
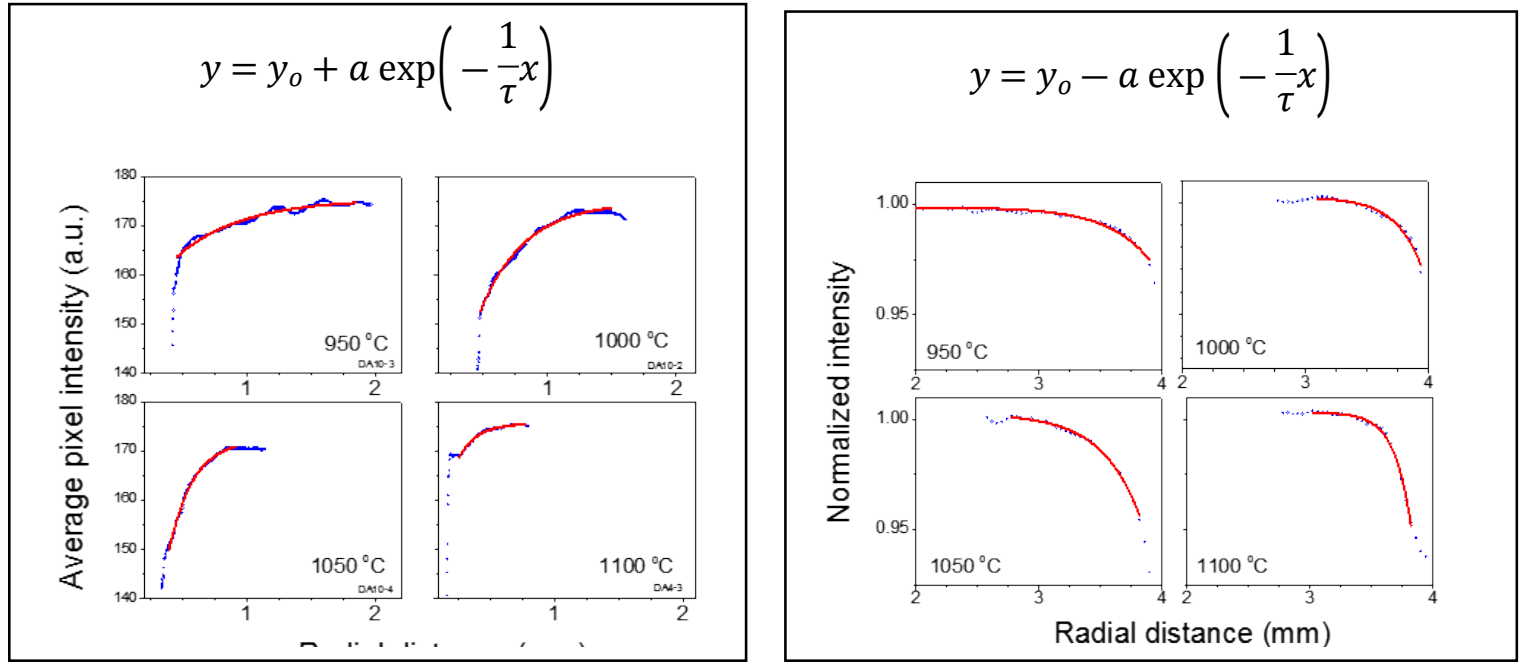

Figure 23. Comparison of fitted density profiles measured by microscopy (left panel) and tomography (right panel) for two halves of the same PCEA specimens oxidized at four temperatures.

\subsection{SIGNIFICANCE}

In this model, we assume that graphite density profiles are the mirror images of oxidant (water) concentration profiles in fully developed oxidized layers obtained at steady-state conditions. Formally, this is recognized by rearranging Eqs. (10) and (30) as follows:

$$
\begin{gathered}
\alpha=-\frac{u_{x}}{U}=\exp \left(-B X_{\alpha}\right) . \\
\alpha=\frac{\Delta \rho_{x}}{\Delta \rho_{\infty}}=\exp \left(-b x_{\alpha}\right) .
\end{gathered}
$$

Validation of the Wichner model is obtained by comparing oxidized layer density profiles, measured by microscopy or tomography, with predicted water concentration profiles calculated with best-fitted kinetic 
and transport parameters. Both profiles, measured and predicted, are expressed by an exponential. The rate of water concentration decay predicted by the Wichner model is $B$, and it connects kinetics and diffusion, Eq. (56). Let $b$ be the rate of density increase in the oxidized layer, Eq. (56). If the theory correctly matches the experiments, both rates should show a common trend with the oxidation temperature and should not differ much from each other. This comparison is discussed in the next section. In addition to the linearized LH model, used for simplification by Wichner, we have also tested full LH and BLH models based on the measurements performed. Specifically, we compared the depth $\left(X_{50 \%}\right)$ predicted for the $\alpha=50 \%$ variation in oxidant concentration from the free gas domain with the depth $\left(x_{50 \%}\right)$ at $50 \%$ change of pore density from direct observations, for each graphite grade, at several oxidation temperatures. 


\section{COMPARISON BETWEEN PREDICTED AND OBSERVED OXIDATION PROFILES}

\subsection{ESTIMATION OF WATER AND HYDROGEN CONCENTRATION PROFILES}

\subsubsection{Basic Equations}

The solutions of decoupled equations for transport of $\mathrm{H}_{2} \mathrm{O}$ and $\mathrm{H}_{2}$ in a long cylinder, at quasi-steady state $(t \rightarrow \infty)$, are found in previous sections. They are repeated here for readers' convenience:

$$
\begin{gathered}
\qquad(r)=u(a) \mathrm{I}_{0}\left(B_{u} r\right) / \mathrm{I}_{0}\left(B_{u} a\right) \text { for water transport. } \\
v(r)=v(a)+\left(B_{v} / B_{u}\right)^{2} \cdot u(r)\left[1-\mathrm{I}_{0}\left(B_{u} r\right) / \mathrm{I}_{0}\left(B_{u} a\right)\right] \text { for hydrogen transport. }
\end{gathered}
$$

These equations describe the concentrations of $\mathrm{H}_{2} \mathrm{O}$ and $\mathrm{H}_{2}$ in a long cylinder as a function of radial distance from the surface, where the cylinder center is at $r=0$ and its surface is at $r=a$. The surface concentrations of $\mathrm{H}_{2} \mathrm{O}$ and $\mathrm{H}_{2}$ can be determined by the free stream concentrations of water $(U)$ and hydrogen $(V)$, the surface mass transfer coefficients for water $\left(h_{u}\right)$ and hydrogen $\left(h_{v}\right)$, and the rate of water diffusion from the surface:

$$
\begin{gathered}
u(a)=\frac{h_{u} U}{h_{u}+D_{u} B_{u}\left[\frac{\mathrm{I}_{1}\left(B_{u^{a}}\right)}{\mathrm{I}_{0}\left(B_{u} u^{a}\right.}\right]} \text { water surface concentration. } \\
v(a)=V+\left(\frac{D_{v}}{h_{v}}\right)\left(\frac{B_{v}{ }^{2}}{B_{u}}\right) u(a) \frac{\mathrm{I}_{1}\left(B_{v} a\right)}{\mathrm{I}_{0}\left(B_{v} a\right)} \text { hydrogen surface concentration. }
\end{gathered}
$$

In these equations, $B_{u}$ and $B_{v}$ connect the rate of oxidation processes (the "sink" for oxidant concentration) with the rate of diffusion (the "spread"),

$$
B^{2}=\frac{A}{D_{e f f}},
$$

where $A$ contains the kinetic term expressing the rate of oxidation estimated at any given condition $\left(P_{\mathrm{H} 20}\right.$, $P_{\mathrm{H} 2}, T$ ) by either the LH or BLH model.

\subsubsection{Linearized LH Model versus Complete Version of LH and BLH Models}

Recall that Wichner used the linearized form of the LH kinetic model, Eq. (3), where $K_{u}$ is the rate constant at the numerator of the full LH rate equation, Eq. (4), and the terms in the denominator were neglected. Moreover, the water partial pressure, $P_{\mathrm{H} 2 \mathrm{O}}=P_{\mathrm{u}}$ was replaced with the gas law equivalent, $\mathrm{R} T$, to obtain a rate equation that depends only on temperature,

$$
A=\text { Rate }_{L H-l i n} \mathrm{RT} \rho N_{u} .
$$

We examined the possibility of using the full rate equations of the LH and BLH models, Eqs. (11) and (12), with the actual parameters for each grade listed previously for the LH model (Table 4) and the BLH model (Table 5). In these "full" versions of the models, the replacement of $P_{\mathrm{H} 2 \mathrm{O}}$ by $R T$ is no longer necessary. However, one needs to normalize Rate $_{L H}$ and Rate $_{B L H}$ by the fraction of exposed carbon atoms in pore surfaces, which are measured by the BET surface area, $S_{\mathrm{BET}}$. If all atoms in 1 gram of carbon were exposed on a flat surface, with gas access on one side only, the surface would be half of the BET surface area of ideal graphene $\left(2630 \mathrm{~m}^{2} / \mathrm{g}\right)$. In reality, the $S_{\mathrm{BET}}$ of nuclear graphite is much lower, about $1-2 \mathrm{~m}^{2} / \mathrm{g}$ 
(see Table 1). With this normalization, the kinetic term of the full LH model, corrected by density and stoichiometric number, becomes

$$
A_{L H}=\text { Rate }_{L H}\left(P_{u}, P_{v}, T\right)=\frac{K_{1} P_{u}}{1+K_{2} P_{v}{ }^{\mathrm{n}}+K_{3} P_{u}}\left(\frac{1530}{S_{B E T}}\right) \rho N_{u},
$$

where the rate constants $K_{i}(i=1,2,3)$ and $n$ are provided in Table 4. The corresponding expression for the full BLH model is

$$
A_{B L H}=\text { Rate }_{B L H}\left(P_{u}, P_{v}, T\right)=\frac{K_{1} P_{u}^{m(T)}}{1+K_{2} P_{v}{ }^{\mathrm{n}}+K_{3} P_{u}^{m(T)}}\left(\frac{1530}{S_{B E T}}\right) \rho N_{u}
$$

with the full expressions of $K_{i}(i=1,2,3), n$ and $m(T)$ provided in Table 5. In these equations, $P_{\mathrm{u}}$ and $P_{\mathrm{v}}$ stand for $P_{\mathrm{H} 2 \mathrm{O}}$ and $P_{\mathrm{H} 2}$.

\subsubsection{Calculation Sequence for Linearized LH Model and Full LH and BLH Models}

The end goal of this section is to evaluate the quasi-steady water concentration profile as a function of temperature for the graphite grades selected for microscopy and tomography characterization, and in the respective oxidation conditions. All calculations were performed using Excel spreadsheets, according to the following steps:

- Define basic properties (density $\rho$, BET surface area $S_{\mathrm{BET}}$, porosity fraction $\varepsilon$, effective diffusivity structural factor $\beta$ ) of selected graphite grades (Table 1 and Table 7).

- Define oxidation conditions (partial pressures in free gas stream of water, $U$, and hydrogen, $V$, temperature, $T$, and diameter, $2 a$ ) of test specimens from each graphite grade (Table 9 ).

- $\quad$ Select the stoichiometric numbers $\left(\mathrm{N}_{\mathrm{u}}=\mathrm{N}_{\mathrm{v}}=1\right.$ for oxidation by water with $\mathrm{CO}$ and $\mathrm{H}_{2}$ as products).

- Specify the internal furnace tube diameter $(22 \mathrm{~cm})$, which is needed for approximating the mass transfer coefficients $h_{u}$ and $h_{v}$.

- Select the kinetic model (linearized LH, full LH, or full BLH) and its proper kinetic parameters (Table 4 for LH model and Table 5 for BLH model).

- Specify reference values for free gas diffusion coefficients: $D_{\mathrm{H} 2 \mathrm{O} / \mathrm{He}}=0.908 \mathrm{~cm}^{2} / \mathrm{s}$ at $25^{\circ} \mathrm{C}$ and 760 Torr; $D_{\mathrm{H} 2 / \mathrm{He}}=1.64 \mathrm{~cm}^{2} / \mathrm{s}$ at $25^{\circ} \mathrm{C}$ and 760 Torr) and the exponents of their temperature variation $\left(q=1.338\right.$ for $\mathrm{H}_{2} \mathrm{O} ; q=1.5$ for $\left.\mathrm{H}_{2}\right)$

- Select an approximation for Nusselt number corresponding to flow conditions in the oxidation tube furnace (at low Reynolds number, use $\mathrm{Nu}=4$ ).

- Calculate, at each temperature, the following:

$\circ$ Effective diffusivity of $\mathrm{H}_{2} \mathrm{O}$ and $\mathrm{H}_{2}$ using Eq. (17).

- Mass transfer coefficient using Eq. (26).

$\circ$ Volume concentration of $\mathrm{H}_{2} \mathrm{O}$ and $\mathrm{H}_{2}$ using ideal gas laws (for linear $\mathrm{LH}$ model).

- Oxidation rates using the appropriate equation for the selected model:

- Linearized LH model: Eq. (20)

- Full LH model: Eq. (4)

- Full BLH model: Eqs. (12) and (12a) 
$\circ$ Volume-normalize (linearized LH) or surface-normalize (full LH and BLH) oxidation rates $\left(A_{u}\right.$ ) as follows:

- Linearized LH model: Eqs. (34a) and (34b).

- For full LH and BLH models, the volume-normalized rates calculated with the respective equations must be corrected by the ratio of exposed surface sites, defined as $S_{\mathrm{BET}} / 1315$, where $S_{\mathrm{BET}}$ is the BET surface area of graphite (Table 1) and the number represents half of the theoretical surface area of graphene $\left(2630 \mathrm{~m}^{2} / \mathrm{g}\right)$.

- Calculate hydrogen generation rates $\left(A_{v}\right)$, which depend on the respective oxidation rates and stoichiometric numbers.

- Calculate oxidation-diffusivity efficiency parameters for $\mathrm{H}_{2} \mathrm{O}\left(B_{u}\right)$ and $\mathrm{H}_{2}\left(B_{v}\right)$ from Eq. (8).

- Calculate surface concentration $(r=a)$ of $\mathrm{H}_{2} \mathrm{O}, u(a)$, and $\mathrm{H}_{2}, v(a)$, from Eqs. (42) and (47).

- Determine by trials the position below the surface $\left(0<X_{\alpha}<a\right)$ where the water concentration drops by a preselected value, $\alpha$, from its surface concentration. This step requires searching for the $X_{\alpha}$ value in Eq. (41) that satisfies the preselected ratio between $u(r)$ and $u(a)$.

- Analyze pixel intensity profiles (using either radius-averaged or smoothed values) or density profiles from microscopy and the respective tomography results, and obtain the best exponential fit using Eq. (55).

- Determine the position below the surface $\left(0<x_{\alpha}<a\right)$ where the pixel intensity (from microscopy) or normalized density (from tomography) is the same fraction $\alpha$ of the bulk value using Eq. (57).

- Compare $\mathrm{X}_{\alpha}$ and $\mathrm{x}_{\alpha}$ at the same temperature and between models.

$\circ$ The graphite density profile is assumed to be the mirror image of water concentration decay.

- If needed, refine calculations by including Knudsen diffusivity $D_{K}$ and calculate global diffusivity coefficients from Eq. (19). In this study, that was done only for grades IG-110 and 2114.

- Calculate detailed water and hydrogen concentration profiles in graphite below the surface, $u(r)$ and $v(r)$, from Eqs. (43) and (49). Results will show an exponential drop inside the graphite of the water concentration from the free gas concentration and hydrogen concentration buildup below the surface. These results are presented in Appendices C, D, E, and F.

- To obtain a complete Wichner model validation, the process detailed above should be repeated for several temperatures and the specific properties and oxidation conditions of specially made specimens.

Section 9.2 presents comparative results for the four graphite grades and between three calculation models: linearized $\mathrm{LH}$, full $\mathrm{LH}$, and full BLH.

\subsection{RESULTS}

Figure 24 shows the temperature dependence of $\mathrm{X}_{50}$, the distance (in $\mathrm{mm}$ ) under the exposed surface at which the models predict a 50\% drop of oxidant (water) concentration from the free gas concentration. All data were calculated at the specific preparation conditions of oxidized specimens for study (Table 9). The curves correspond to the three models: linearized LH, full LH, and full BLH. Variants of the full LH and BLH models corrected for the Knudsen diffusion are also shown. On the same images, the symbols show depths for a $50 \%$ deviation in average porosity and density from the centers of specimens. These data were calculated from exponential fitting of optical microscopy pixel intensities and normalized tomography densities (only for PCEA and NBG-17).

Several observations are appropriate:

- Each model shows, as expected, that the penetration depth increases as the oxidation temperature decreases. 
- There are clear differences between the models. The linearized LH model almost always predicts a deeper penetration of oxidant.

- The agreement between direct observations and model predictions is very good for the medium-fine graphite grades (PCEA and NBG-17). Between 950 and $1100^{\circ} \mathrm{C}$ (PCEA) and 900-1050 ${ }^{\circ} \mathrm{C}$ (NBG17), the full LH and BLH models agree with each other and reproduce well the microscopy and tomography results.

- The linearized LH model fails to fit data for superfine grades IG-110 and 2114. Of the two other models, the full BLH model (and to a lesser extent the full LH model) reproduce the microscopy result at $1050^{\circ} \mathrm{C}$ but deviate at lower temperatures.

- When a correction for Knudsen diffusivity was applied to the full LH and BLH models, they showed better correlation with 2114 microscopy results over a broader temperature range $\left(950-1050{ }^{\circ} \mathrm{C}\right)$. The effect of Knudsen correction was to moderate the temperature variation of $D_{\text {eff, }}$ which resulted in less penetration.

- Superfine grade IG-110 was systematically at variance with model predictions. Microscopy results failed to detect the development of new porosity near the surfaces of all specimens, even after oxidation at $4-5 \mathrm{wt} \%$. This is proved again by the polarized light images in Figure 25. There might be several reasons for that outcome:

$\circ$ There is significant closed porosity in IG-110 (which correlates with its very low He permeability). Closed porosity limits penetration. However, there were no detectable dimensional changes after oxidation, negating the hypothesis of intense, surface-only oxidation

- Newly developed porosity (by oxidation) should have pore sizes very similar to the already existing pores in IG-110. These new pores could not be differentiated (by microscopy) from the normal porosity of IG-110. However, high-magnification images $(1000 \times)$ did not detect microstructural differences between the center and the near-surface zones in oxidized IG-110. $\circ$ Oxidation occurred deep inside the specimens. The $4 \mathrm{~mm}$ diameter was totally penetrated by oxidation, as the LH model predicts (1.5-2 $\mathrm{cm}$ in Figure 24). This hypothesis is supported by German data on IG-110 oxidation by steam. ${ }^{10}$ At 3 bar total pressure (rather than 1 bar in our experiments) and $1000{ }^{\circ} \mathrm{C}$, oxidation at $P_{\mathrm{H} 2 \mathrm{O}}=474 \mathrm{mbar}=47,400 \mathrm{~Pa}$ caused an exponential density decay over $3 \mathrm{~mm}$ at $11.5 \%$ total burn-off. 

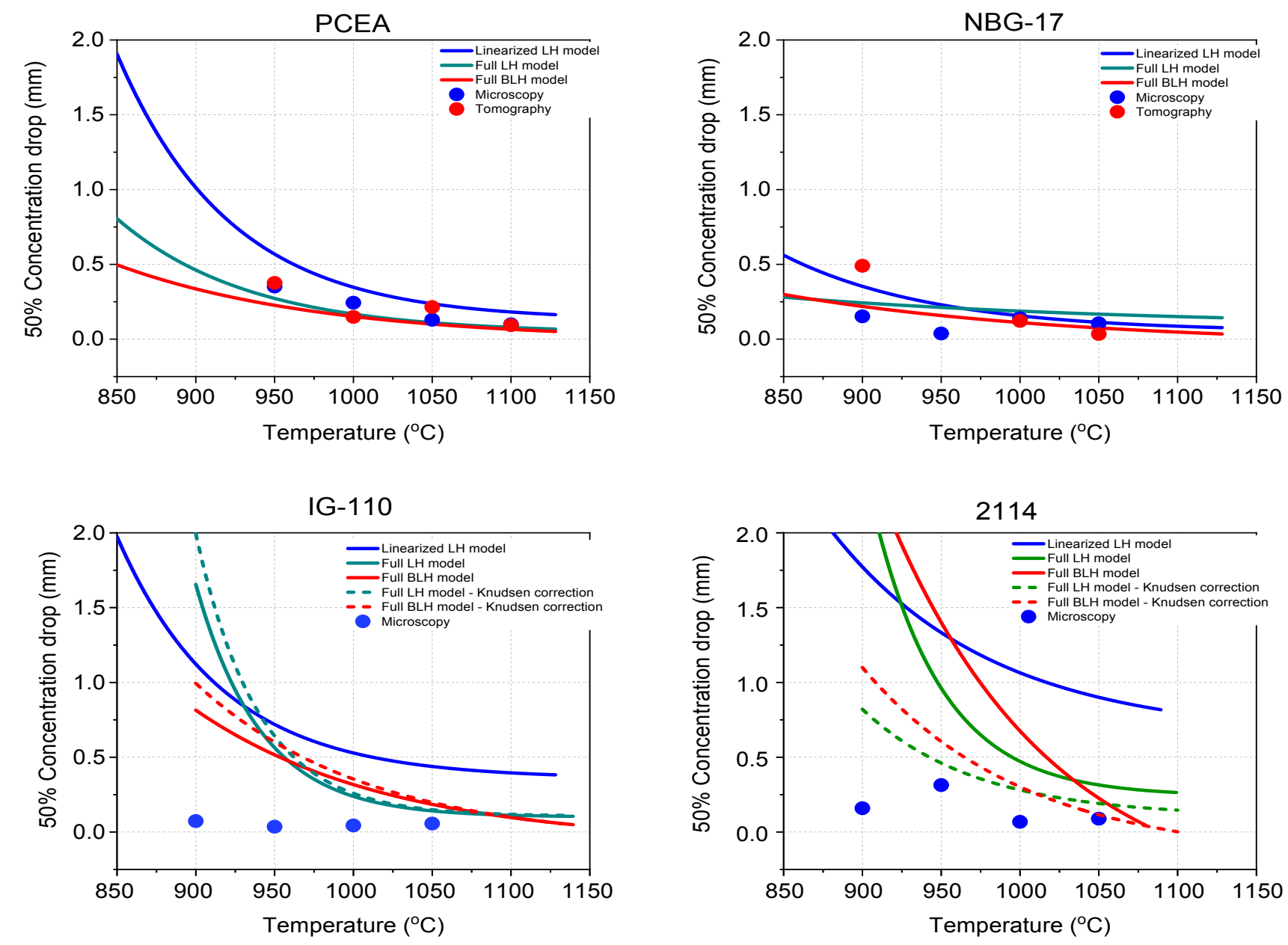

Figure 24. Comparison among predictions of various models for penetration depth at $50 \%$ oxidant concentration drop and experimental results at $\mathbf{5 0 \%}$ graphite density drop for specimens oxidized at various temperatures 

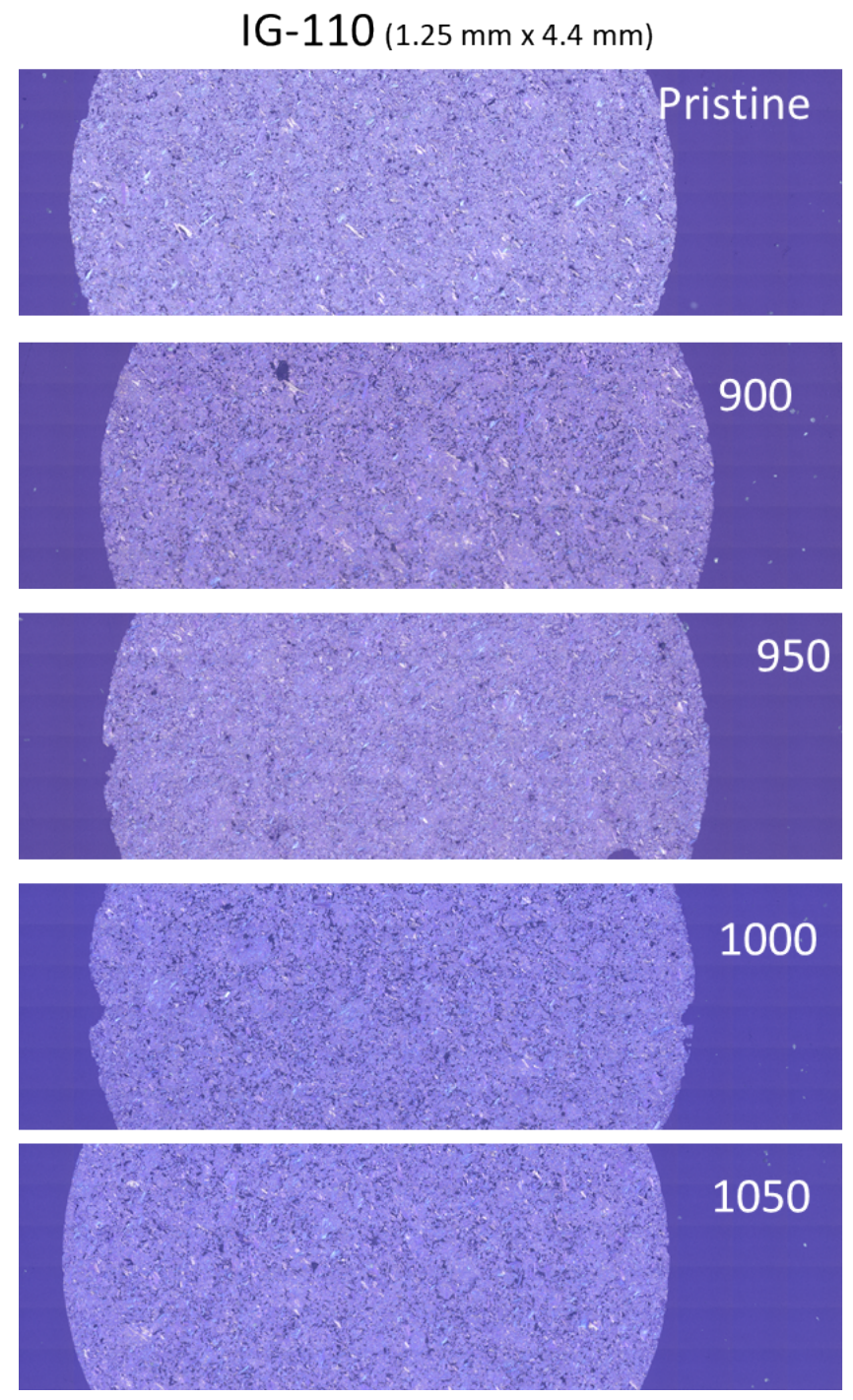

Figure 25. Polarized light microscopy images of pristine and oxidized graphite IG-110, showing lack of surface-localized oxidized layer 


\section{SUMMARY AND CONCLUSIONS}

The need to predict the long-time corrosion effect of water impurities in the He coolant of HTGRs during normal operating conditions has prompted modeling efforts in the United States, Germany, China, and Japan. The theoretical grounds of this effort are more or less clear: they involve computational analysis that combines flow, temperature, and concentration conditions in the free gas domain, heat and mass transfer at the solid/gas interface and in the porous graphite body, and chemical kinetics describing the variation of spatial and temporal oxidant concentration profiles in porous graphite at the locus of oxidation reactions. Chronic oxidation is a complex process, driven by the balance between oxidant diffusion rates and oxidant consumption rates by chemical reaction; the latter is slowed by hydrogen generated as a reaction product. Chemical kinetics is traditionally expressed by the classical LH rate equation. Past modeling efforts were made for grand sections of HTGR reactors, ${ }^{30}$ or to only one graphite channel, ${ }^{58,59}$ and even smaller details, ${ }^{60,61,61,63}$ or, in a general way, to the local gas-solid interface. ${ }^{2,64}$ Modeling employed complex computer codes and engineering applications such as COMSOL. All results have shown repeatedly that the long-term damage caused by chronic graphite oxidation will be limited to a small layer at the exposed surface of the graphite fuel elements at the reactor bottom, where the temperature is higher.

Penetration depth and oxidized layer profiles after chronic oxidation are critical factors that determine the geometry of cooling channels in prismatic HTGR fuel elements. Correct evaluation of the amount and localization of graphite corrosion by chronic oxidation is critical for nuclear safety analysis. Small burnoff, but spread uniformly through the volume during the reactor's lifetime, is more damaging for mechanical strength than more severe but surface-localized oxidation.

The quality of results mirrors the quality of the primary information fed to the models. Here there still are a few unknowns, which we tried to uncover. The most important are the numerical parameters of the kinetic models and even the choice of the optimal model itself. Extrapolations and approximations are part of the discovery process, and they could not be totally avoided. It is clear that attempting oxidation rate measurements at the real conditions in normal operating HTGRs, even though they could be reproduced in the lab, would have been an extremely slow and impractically daunting task, at extremely large costs. Using accelerated oxidation tests is an acceptable procedure. The rationale is based on the assumption that the kinetic model has been validated on a range of various conditions, and the results could be safely extrapolated back to the extremely "dry" conditions of the HTGR core. On the other hand, every graphite grade is different because its microstructure is unique. Regulatory bodies demand proper evidence that every material in the reactor core was properly characterized in conditions relevant for normal operating and transient conditions. This motivates the studies presented here. Similar studies are in progress in other research groups.

Our detailed kinetic studies of accelerated graphite oxidation in an $\mathrm{H}_{2} \mathrm{O} / \mathrm{He}$ mixture fills a significant knowledge gap. We selected four graphite grades that are considered possible candidates for HTGR core components: two medium-fine grades (PCEA, NBG-17) and two superfine grades (IG-110, 2114). After analyzing several hundreds of experimental observations for each grade, we discovered that the full version of the LH model (six parameters) is generally suitable for medium-grain graphite within limited windows of conditions (low water partial pressures and temperatures). However, results for superfine grades were less accurately fit by the full LH model, especially at higher water vapor pressures and temperatures. To improve the fitting for all grades over broader ranges of pressure and temperature, we introduced a modification in the LH model. The full version (ten parameters) of the newly developed BLH mode ${ }^{15}$ provides reliable results for oxidation rates varying by more than three orders of magnitude, variations of $P_{\mathrm{H} 2 \mathrm{O}}$ by almost 1000 times, and a $350{ }^{\circ} \mathrm{C}$ variation of $T$. It works for all four grades of graphite. On the other hand, we have shown that a simplified (linearized) version of the LH 
equation (two parameters) is a rough approximation of experimentally observed trends, while an equivalent linearized BLH version is not usable. In previous reports, we have presented complete lists of all kinetic observations. ${ }^{24,26,28}$ The corresponding parameters of the $\mathrm{LH}$ and BLH models are listed in Table 4 and Table 5.

A second unknown property needed for model development is the appropriate value of the structural factor $\beta=D_{e f f} / D_{A B}$ that defines moisture effective diffusivity in every graphite grade. Prior models have either used an approximate value $(\beta=0.01)$ derived from old diffusivity measurements made by British engineers on their graphite grades, or attempted to evaluate $\beta$ from structural parameters, of which some are difficult to measure (tortuosity). With fresh diffusivity measurements from $\mathrm{H}_{2} \mathrm{O} / \mathrm{He}$ mixtures on the same four graphite grades, we found that modern grades are characterized by much lower $\beta$ factors (in the $10^{-3}$ range). This discovery was independently confirmed by our INL colleagues. ${ }^{44}$ Because of reduced pore channels in higher-density modern graphites, the Knudsen diffusion may have a higher importance than previously thought because it slows down normal (viscous) diffusion in superfine grain grades. Knudsen diffusion is temperature dependent but in a different way from normal diffusion.

The model for chronic oxidation of graphite developed by Wichner introduces a series of simplifying assumptions, which in the end made possible elegant analytical solutions for reasonable predictions that otherwise would have required heavy numerical analysis and specialized computational efforts. The main assumptions, listed here again, were (1) a focus on steady-state solution at long oxidation times, (2) linearization of the rate equation, (3) neglect of graphite density variations, and (4) constancy of diffusion parameters. With that, the objective was assessment of oxidant penetration profiles and of their mirroring the graphite density variation in the oxidized layer. The main information needed for this task is knowledge of "true" oxidation rates and of the proper effective diffusivity coefficients. Figure 1Figure 26 is an updated version of Figure 1, showing how various groups of results were incorporated in the Wichner model and used for its validation. The kinetics was represented by the three models discussed above. The temperature effects on the relationship between kinetics and transport were tested by comparing predicted water vapor concentration profiles with measured graphite density and porosity trends in graphite with enhanced contrast in the oxidized layer. Several samples, oxidized slowly by longtime exposure in $\mathrm{H}_{2} \mathrm{O} / \mathrm{He}$ mixtures at four temperatures, were produced to observe the fully developed oxidized layers. They were analyzed by optical microscopy and (only for medium-fine grades) by x-ray tomography (courtesy of our INL colleagues). The density profiles of fine pores observed microscopically, and the normalized density from tomography, were fitted with the same type of exponential function that describes predicted water vapor concentration decay under the surface. The results were compared at an arbitrarily selected 50\% drop in density and concentration. The temperature trends were the same, meaning that penetration was higher at lower temperatures, as expected. However, there were differences between the models and between graphite types. 


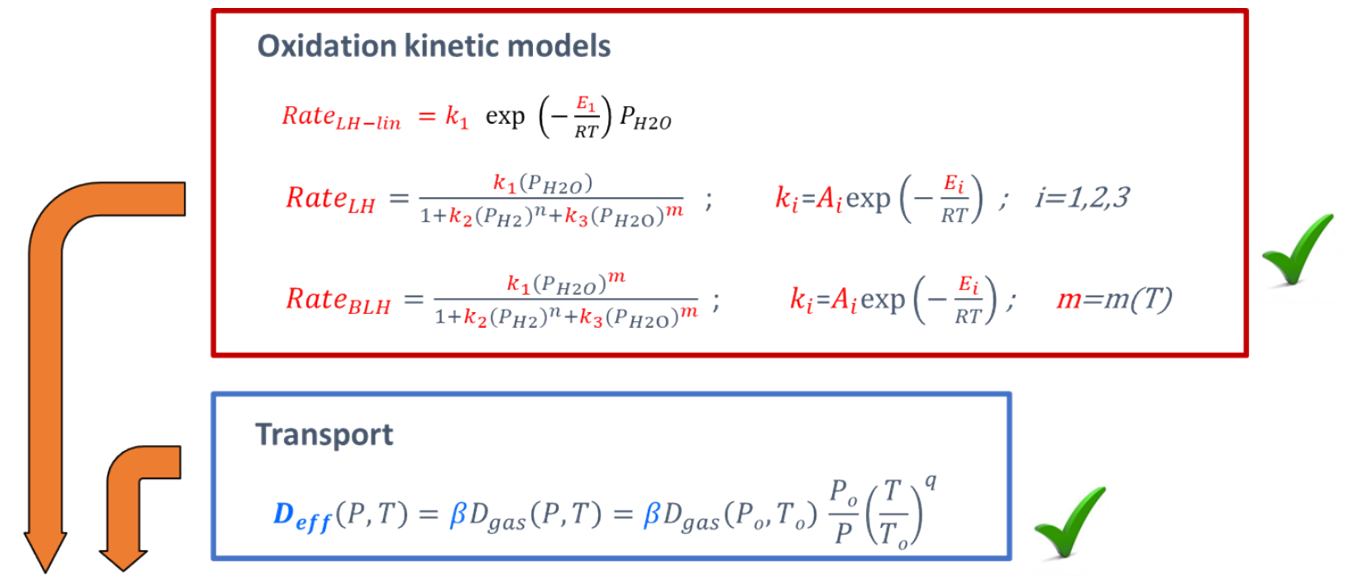

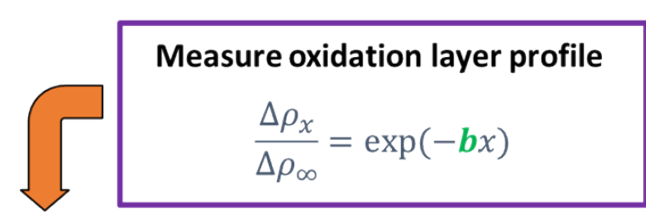

Compare and validate models

LH-linear < full LH < full BLH

Figure 26. Updated schematic of experimental strategy showing correlations between various groups of results

The linearized LH model used by Wichner (with two parameters only) was an approximation convenient for calculations, but it did not fit well the data. We explored the use of full versions of the LH and BLH models (with six and ten parameters, respectively). In general, the full BLH model performed better than the full LH model, but only for three graphite grades (medium-fine PCEA and NBG-17 and superfine 2114). After the introduction of an idealized Knudsen diffusion coefficient, the match between model predictions and density and porosity drop was greatly improved for grade 2114 . But no agreement could be found for IG-110, and the explanations provided above may not be satisfactory.

The accomplishments of this research project can be summarized in a few lines:

- Performed and published detailed kinetic analysis for chronic oxidation by moisture of four graphite grades with medium-fine and superfine grain structures.

- Verified the limited applicability of the LH model and developed the modified BLH for better fitting of observables.

- Provided and published full sets of numerical values of kinetic parameters corresponding to LH and BLH models.

- Estimated the effective diffusivity of water from $\mathrm{H}_{2} \mathrm{O} / \mathrm{He}$ mixtures and obtained averaged values of the structural $\beta$ scaling parameters of each graphite grade.

- Analyzed density profiles and determined points of 50\% density and porosity drop near the surface of graphite specimens oxidized at 5-6 wt \% loss at different temperatures. 
- Using the mathematical apparatus built around the Wichner model for chronic oxidation of graphite, predicted the moisture concentration drop at steady state conditions as a function of temperature for each grade of graphite, at experimental conditions selected to match the preparation of highly oxidized specimens. These calculations were made using the functions built into Excel spreadsheets.

- Comparing predictions against observations on oxidized layers, found that the full BLH model provided the best fitting, followed closely by the full LH model (especially when Knudsen diffusion was incorporated for the superfine 2114 grade). However, agreement was not found for the superfine grade IG-110, which did not show any contrast associated with the oxidized layer.

Finally, we noticed with satisfaction that, a short time after publication of the LH and BLH data ${ }^{29}$ for the four graphite grades in 2018, they were used in a 2019 publication ${ }^{30}$ by joint authors from the University of Michigan, Ohio State University, and University of Idaho. Their scope, which was broader than ours, was full-scale multiphysics modeling of the chronic oxidation effects of the same graphites after 36 months of operation in a modular HTGR. The authors had back-extrapolated and compared the LH and BLH models, and eventually decided to use the LH model. They selected operating conditions in $\mathrm{He}$ coolant with $1.2 \mathrm{~Pa} \mathrm{H}_{2} \mathrm{O}$ and $10 \mathrm{~Pa} \mathrm{H}_{2}$ at temperatures between 800 and $1400 \mathrm{~K}$ and $6.4 \mathrm{MPa}$ total pressure. Simulations made by the COMSOL Multiphysics package accounted for fluid flow, mass and heat transfer, chemical reactions, and structural changes at various depths in a full-scale graphite coolant channel (7.8 $\mathrm{m}$ long). The results showed higher oxidation levels at the channel bottom (where the temperature is higher) and different behaviors among the graphite grades. The latter are caused by differences in kinetic and transport properties. The paper emphasized the importance of obtaining highly accurate experimental rate information at low partial water vapor pressure, preferably $\mathrm{P}_{\mathrm{H} 2 \mathrm{O}}<10 \mathrm{~Pa}$. 


\section{REFERENCES}

1. P L Walker, F Rusinko, L G Austin, Gas reactions of carbon, Advances in Catalysis 11 (1959) 133221.

2. M B Richards, Reaction of nuclear grade graphite with low concentrations of steam in the helium coolant of an MHTGR, Energy 15 (1990) 729-739.

3. T D Burchell, Next Generation Nuclear Plant Phenomena Identification and Ranking Tables (PIRT), vol. 5. Graphite PIRTs, NUREG/CR-6944, vol. 5 (2007).

4. N C Gallego and T D Burchell, Comparison of NRC Graphite PIRT and DOE Planned Research Activities for Graphite, ORNL/NRC/LTR-09-01 (2009).

5. R P Wichner, T D Burchell, C I Contescu, Note on Graphite Oxidation by Oxygen and Moisture, ORNL/TM-2008/230, 2008.

6. R P Wichner, T D Burchell, C I Contescu, Penetration depth and transient oxidation of graphite by oxygen and water vapor, J Nucl Mater 393 (2009) 518-521.

7. C I Contescu, S Azad, D Miller, M J Lance, F S Baker, T D Burchell, Practical aspects for characterizing air oxidation of graphite, J Nucl Mater 381 (2008) 12-24.

8. C I Contescu, T Guldan, P Wang, T D Burchell, The effect of microstructure on air oxidation resistance of nuclear graphite, Carbon 20 (2012) 3354-3366.

9. P Wang, C I Contescu, S Yu, T D Burchell, Pore structure development in oxidized IG-110 nuclear graphite, J Nucl Mater 430 (2012) 229-238.

10. H-K Hinsen, W Katscher, K-J Loenissen, Experimental investigation of corrosion of IG-110 graphite by steam, KFA Jul-Spez-578, July 1990, ISSN 0343-7639 (Forschungszentrum Julich GmbH).

11. C I Contescu, Chronic oxidation of nuclear graphite, Talk at 6th Annual Technical Review Meeting, VHTR Technology Development Office, Idaho Falls, May 9, 2013.

12. C I Contescu, Graphite oxidation: Analysis and modeling results, presented at the Advanced Materials R\&D Program Review, organized by DOE-NE Advanced Reactor Technologies Program, Oak Ridge, TN, May 6-8, 2014.

13. C I Contescu, Graphite oxidation: Recent results, presented at the Advanced Reactor Technologies, Advanced Materials R\&D Program Review, organized by DOE-NE, Idaho Falls, ID, July 14-15, 2015.

14. C Contescu, Graphite oxidation model development, Advanced Materials Program Review, Advanced Reactor Technologies, Department of Energy-Nuclear Energy Office, Germantown, MD, June 7-8, 2016.

15. C Contescu, Chronic graphite oxidation: Recent results, Advanced Reactor Technologies, Advanced Materials Program Review, DOE NE Office Building, Germantown, MD, June 6-7, 2017.

16. C I Contescu, R W Mee, T D Burchell, Kinetics of chronic oxidation of PCEA nuclear graphite by moisture in helium coolant, presentation at the GEN IV $8^{\text {th }}$ Official Graphite Working Group, Japan Atomic Energy Agency (Oarai-Ibaraki, Japan; April 14-15, 2014).

17. C Contescu, J Kane, R Mee, A Bontorno, N Gallego, T Burchell, W Windes, Reactivity differences between graphite grades during long time exposure in the helium coolant, INGSM-16, International Nuclear Graphite Specialists Meeting (Nottingham, UK, September 13-17, 2015). 
18. C Contescu, R Mee, J Kane, N Gallego, T Burchell, W Windes, Predictive model for chronic oxidation of nuclear graphite during HTGR operation, CARBON 2016, The World Conference on Carbon, Penn State University, State College, PA (July 10-15, 2016). (Proceedings in electronic form)

19. C I Contescu, R W Mee, N C Gallego, J Kane, T D Burchell, W Windes, Beyond classical kinetic models for graphite oxidation by moisture in helium coolant, INGSM-17 (International Nuclear Graphite Specialists Meeting), International Atomic Energy Agency, Vienna, Austria (September 58,2016 ) (Proceedings distributed in electronic form)

20. C Contescu, R Mee, J Kane, N Gallego, T Burchell, W Windes, Kinetic models for oxidation of nuclear graphite by moisture in helium coolant, ROMPHYSCHEM 16 (Romanian Conference of Physical Chemistry, $16^{\text {th }}$ edition), Galati, Romania (September 21-23, 2016).

21. Y Lee, C I Contescu, R W Mee, Chronic oxidation of Mersen 2114 fine grained nuclear graphite by water vapor in normal HTGR operations, International Nuclear Graphite Specialists Meeting (INGSM-2017), Baltimore, MD, September 17-21, 2017 (oral). (Proceedings distributed in electronic form.)

22. C I Contescu, T D Burchell: Initial Tests for Determination of Nuclear Graphite Oxidation Kinetics in Conditions Relevant to VHTR Normal Operation, ORNL/TM-2011/472 (December 2012).

23. C I Contescu, T D Burchell, Research Needs and Test Plan for Characterization of Nuclear Graphite Oxidation, ORNL/TM-2012/263 (August 2012).

24. C I Contescu, T D Burchell, R W Mee, Accelerated Oxidation Studies of PCEA Nuclear Graphite by Low Concentration of Water and Hydrogen in Helium, ORNL/TM-2013/524 (November 2013).

25. C. I. Contescu, T. D. Burchell, Water Vapor Transport in Nuclear Graphite, ORNL/TM-2015/88 (March 2015).

26. C I Contescu, T D Burchell, R Mee, Kinetics of Chronic Oxidation of NBG-17 Nuclear Graphite by Water Vapor, ORNL/TM-2015/142 (May 2015).

27. C I Contescu, R W Mee, Status of Chronic Oxidation Studies of Graphite, ORNL/TM-2016/195 (May 2016).

28. C Contescu, Y Lee, R Mee, Oxidation Kinetics by Water Vapor of Nuclear Graphite Grade 2114, ORNL/TM-2018/1057 (November 2018).

29. C I Contescu, R W Mee, Y Lee, J D Arregui-Mena, N C Gallego, T D Burchell, J Kane, W E Windes, Beyond the classical kinetic model for chronic graphite oxidation by moisture in high temperature gas-cooled reactors, Carbon 127 (2018) 158-169; corrigendum: Carbon 140 (2018) 249.

30. C Wang, X Sun, R N Christensen, Multiphysics simulation of moisture-graphite oxidation in MHTGR, Annals Nucl Energy 131 (2019) 483-495.

31. D J Baker, J B Morris, Structural damage in graphite occurring during pore size measurements by high pressure mercury, Carbon 9 (1971) 687-690.

32. M P Kissane, A review of radionuclide behavior in the primary system of a very-high temperature reactor, Nucl Eng Des 239 (2009) 3076-3091.

33. $\mathrm{X} \mathrm{Yu,} \mathrm{S} \mathrm{Yu}$, Analysis of fuel element matrix graphite corrosion in HTR-PM for normal operating conditions, Nucl Eng Des 240 (2010) 738-743.

34. E R Corwin, Generation IV Reactors Integrated Materials Technology Program Plan: Focus on Very High Temperature Reactor Materials, ORNL/TM-2008/129 (2008). 
35. M Fechter, Graphite oxidation modeling for a $250 \mathrm{MW}$ pebble bed reactor, Oak Ridge National Laboratory presentation, July 2010.

36. B Castle, NGNP Reactor Coolant Chemistry Control Study, INL/EXT-10-10533.

37. R N Wright, Kinetics of Gas Reactions and Environmental Degradation in NGNP Helium, INL/EXT06-11494 (2006).

38. C Velasquez, G Hightower, R Burnette, The Oxidation of H-451 Graphite by Steam. Part 1: Reaction Kinetics, General Atomic Company, GA-A14951 (1978).

39. J Gadsby, C N Hinshelwood, K W Sykes, The kinetics of the reactions of the steam-carbon system, Proc Roy Soc (London) A, 187 (1946) 129-151.

40. R C Giberson and J P Walker, Reaction of nuclear graphite with water vapor. Part I. Effect of hydrogen and water vapor partial pressures, Carbon 3 (1966) 521-525.

41. R C Giberson and J P Walker, Reaction of nuclear graphite with water vapor. Part I. Effect of hydrogen and water vapor partial pressures, Carbon 3 (1966) 521-525.

42. E N Fuller, P D Schettle, J C Giddings, A new method for prediction of binary gas-phase diffusion coefficients, Ind Eng Chem 58 (1966) 19-35; extracted from Landolt-Bornstein IV/15A, Chapter 5.1.1: Diffusion of Gas/Vapor in Gas.

43. C M Luijten, $\mathrm{K} J$ Bosschaart, M E H van Dongen, A new method for determining binary diffusion coefficients in dilute condensable vapors, Intl J Heat Mass Transfer 40 (1997) 3497-3502.

44. J Kane, A C Matthews, C J Orme, C I Contescu, W D Swank, W E Windes, Effective gaseous coefficients of select ultra-fine, super-fine and medium grain nuclear graphite, Carbon 136 (218) 369379.

45. R J Millington, J M Quirk, Permeability of porous solids, Trans Faraday Soc 57 (1961) 1200-1207.

46. G F Hewitt, E W Sharratt, Gaseous diffusion in porous media with particular reference to graphite, Nature 198 (1963) 952-957.

47. R B Evans, G M Watson, E A Mason, Gaseous diffusion in porous media at uniform pressure, J. Phys. Chem. 35 (1961) 2076-2083.

48. D Mu, X-S Liu, C Huang, N Djilali, Determination of the effective diffusion coefficient in porous media including Knudsen effects, Microfluid Nanofluid 4 (2008) 257-260.

49. PMI Porous Materials, https://www.pmiapp.com/ (accessed September 12, 2019).

50. E Wicke, Untersuchungen uber Ad- und Desorptionsvorgange in kornigen, durchstrromtem Adsorberschichten, Kolloid-Zeitschrift 93 (1940) 129-157.

51. A Wheeler, Reaction rates and selectivity in catalyst pores, Adv Catalysis 3 (1951) 260-275.

52. P B Weisz, C D Prater, Measurements in experimental catalysis, Adv Catalysis 6 (1951) 185-195.

53. P L Walker, F Rusinko, E Raats, Anisotropy of effective gas diffusion coefficients in extruded carbon rods, Nature 176 (1955) 1167.

54. C Y Lee, C R Wilke, Measurements of vapor diffusion coefficient, Ind Eng Chem 46 (1954) 23812387.

55. T J Gerczak, Oxidation of matrix material in helium with varied moisture content, Gas-Cooled Reactor Fuels and Methods Program Review, June 18-19, 2019, Idaho Falls, ID.

56. J Lee, T Gerczak, R Mee, C Contescu, Kinetic studies on chronic and acute oxidation by water of matrix carbon for nuclear fuel, CARBON 2019, Lexington, KY, July 14-19, 2019. 
57. C I Contescu, Effect of air oxidation on pore structure development and mechanical properties of nuclear graphite, ORNL/TM-2010/197 (September 2010).

58. X Yu L Brissonneau, C Bourdeloie, S Yu, The modeling of graphite oxidation behavior for HTGR fuel coolant channels under normal operating conditions, Nucl Eng Design 238 (2008) 2230-2238.

59. C Wang, X Sun, R N Christensen, S Shi, Numerical simulations of chronic graphite oxidation in MHTGR, Trans American Nuclear Soc 118 (2018) 723-726.

60. X Luo, J-C Robin, S Yu, Theoretical analysis of mass-transfer and reaction in a porous medium applied to the gasification of graphite by water vapor, Nucl Eng Design 236 (2006) 938-947.

61. K Katscher. W Delle, H K Hinssen, R Moormann, E Wallura, Changes in the macroporosity of nuclear reactor graphite under oxygen corrosion, High Temp High Press 13 (1981) 275-279.

62. X Yu, S Yu, Analysis of fuel element matrix graphite corrosion in HTR-PM for normal operating conditions, Nucl Eng Design 240 (2010) 738-743.

63. X Luo, X Yu, S Yu, J-C Robin, Analysis of graphite gasification by water vapor at different conversions, Nucl Eng Design 273 2014) 68-74.

64. H E Kim, C H Oh, H C No, Experimental study and model development on the moisture effect for nuclear graphite oxidation, Nucl Technol 164 (2008) 278-285. 


\section{APPENDIX A. DEFINITIONS AND CONSTANTS}

\section{Definitions}

Oxidation rate (linearized)

Stoichiometric number

Rox mol_Cox / (mol_C.s)

Distance from surface ( $x=0$ at surface)

$\mathrm{N}$ mol_oxidant/mol_C_oxidized

\section{Formulae}

Oxidation rate (volumic) linearized $\mathrm{LH}$

Oxidation rate (volumic) LH full

Oxidation rate (volumic) BLH full

Reaction order exponent in BLH equation

Oxidation efficiency factor

Diffusion coefficient variation

Temperature exponent for $D(T, P)$

Penetration depth (equilibrium)

Fractional concentration drop

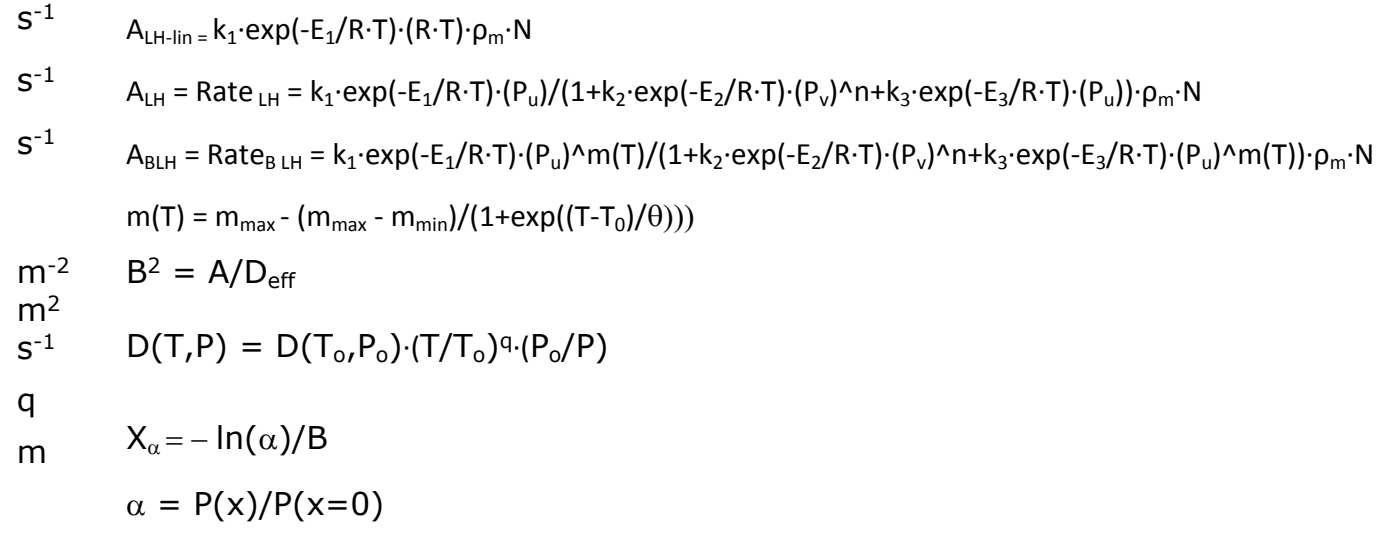

\section{Constants}

Molecular weight C

Gas constant

Diffusion coefficient $\mathrm{H} 2 \mathrm{O} / \mathrm{He}$

Temperature exponent for $\mathrm{D}_{\mathrm{H} 2 \mathrm{O} / \mathrm{He}}$ Diffusion coefficient $\mathrm{H} 2 / \mathrm{He}$

Temperature exponent for $D_{\mathrm{H} 2 / \mathrm{He}}$
Mw $12 \mathrm{~mol} / \mathrm{g}$
R $\quad 8.314 \mathrm{~J} / \mathrm{mol} \mathrm{K}$
$D_{u} \quad 9.08 \mathrm{E}-05 \mathrm{~m} 2 / \mathrm{s}$ at $1 \mathrm{bar}, 298 \mathrm{~K}$
$\mathrm{q}_{\mathrm{u}} \quad 1.338$
Dv $\quad 1.64 \mathrm{E}-04 \mathrm{~m} 2 / \mathrm{s}$ at 760 Torr, $298 \mathrm{~K}$
$\mathrm{q}_{\mathrm{v}} \quad 1.5$ 



\section{APPENDIX B. GRAPHITE PROPERTIES}

\section{Graphite properties}

Bulk (apparent) density (Archimedes) Skeletal density (He pycnometry)

Assumed ideal skeleton density

Total pore volume

Volume of $1 \mathrm{gram}$

Total Porosity fraction

Molar(bulk) density

BET surface area

Fraction of surface atoms

Kinetics - oxidation by water

\section{LH model}

Pre-exponent in LH equation Activation energy in $\mathrm{BLH}$ equation

Pre-exponent in LH equation

Activation energy in $\mathrm{L}-\mathrm{H}$ eq.

Pre-exponent in $\mathrm{LH}$ equation

Activation energy in $\mathrm{L}-\mathrm{H}$ eq.

Kinetic order for $\mathrm{H}_{2}$

\section{PCEA}

$\rho_{\text {ap }}$

$\rho_{\text {sk }}$

$\rho_{\text {ideal }}$$$
\varepsilon
$$$$
\rho_{\mathrm{m}}
$$$$
S_{\mathrm{BET}}
$$$$
S_{\mathrm{BET}} / 1315
$$

\begin{tabular}{|c|}
\hline $\mathrm{g} / \mathrm{cm}^{3}$ \\
\hline $\mathrm{g} / \mathrm{cm}^{3}$ \\
\hline $\mathrm{g} / \mathrm{cm}^{3}$ \\
\hline $\mathrm{cm}^{3} / \mathrm{g}$ \\
\hline $\mathrm{cm}^{3}$ \\
\hline $\mathrm{mol} \mathrm{C} / \mathrm{cm}^{3}$ \\
\hline $\mathrm{m}^{2} / \mathrm{g}$ \\
\hline
\end{tabular}

1.809

1.912

2.25

0.108

0.553

0.196

0.151

1.547

0.001

PCEA

$5.90 \mathrm{E}-01$
199000
$5.40 \mathrm{E}+09$
280000
$4.00 \mathrm{E}-04$
-39000

3.85E-06

4.00E-08

$-186561$

5.79E-07

$-123000$

0.5
61500

IG-110

2114
1.854
2.100

1.779

1.937

2.27

0.122

0.562

0.216

0.148

1.245

0.001

IG-110

$\begin{array}{rr}8.29 \mathrm{E}-06 & 6.89 \mathrm{E}-07 \\ 86000 & 57000 \\ 4.18 \mathrm{E}-08 & 2.56 \mathrm{E}-03 \\ -193000 & -23922 \\ 6.29 \mathrm{E}-11 & 4.91 \mathrm{E}-09 \\ -211000 & -167000\end{array}$

0.5

0.5
2114

1.815

2.029

2.27

0.110

0.551

0.200

0.151

1.178

0.001

0.5 


\section{Graphite properties}

Pre-exponent in BLH equation Activation energy in BLH equation

Pre-exponent in BLH equation Activation energy in BLH equation Pre-exponent in BLH equation

Activation energy in BLH equation

Hydrogen kinetic order

Upper limit of water kinetic order

Lower limit of water kinetic order

Characteristic temperature

Scaling factor

\section{Water transport}

Graphite structural factor

$$
\pm \text { error interval }
$$

D effective measured $\left(35^{\circ} \mathrm{C}, \mathrm{P}\right.$ atm)

Helium permeability

\section{PCEA}

$\mathrm{Pa}^{-\mathrm{m}} \mathrm{s}^{-1}$

$\mathrm{J} / \mathrm{mol}$

$\mathrm{Pa}^{-0.5}$

$\mathrm{J} / \mathrm{mol}$

$\mathrm{Pa}-\mathrm{m}$

$\mathrm{J} / \mathrm{mol}$

$E_{3}$

$m_{\max }$

$m_{\text {min }}$

$T_{0}$

$\theta$

K

K
NBG-17

IG-110

5.93E-02

161700

$2.1 \mathrm{E}+05$

166800

$1.43 \mathrm{E}-15$

$-292600$

0.5

0.638

0.445

1283

10.8

PCEA

$\beta=D_{\mathrm{eff}} / D_{\mathrm{AB}}$

$D_{\text {eff }}$

$\kappa$
4.35E-08
11400
3.92E-06
$-121700$
1.25E-10
$-203200$
0.5
0.932
0.228
1275
35.5

2.83E-03

5.10E-04

1.94E-03

2.58E-03

6.10E-04

$\mathrm{cm}^{2} / \mathrm{s}$

$\mathrm{m}^{2}$

1.90E-03

4.00E-14

7.00E-13
7.64E-12

$-70500$

$1.88 \mathrm{E}-02$

$-39600$

6.07E-18

$-373500$

0.5

1.55

$-0.070$

1326

64.2

NBG-17 IG-110

2114

2.69E-03

2.10E-04

9.10E-03

2.46E-03

4.20E-04

2114

9.13E-12

$-61400$

$1.48 \mathrm{E} 00$

1650

3.38E-14

$-280600$

0.5

1.46

0.063

1288

39.8

$6.50 \mathrm{E}-15$

8.31E-03

$6.00 \mathrm{E}-13$ 
APPENDIX C. WATER PENETRATION AND HYDROGEN BUILDUP PROFILES

IN GRAPHITE PCEA

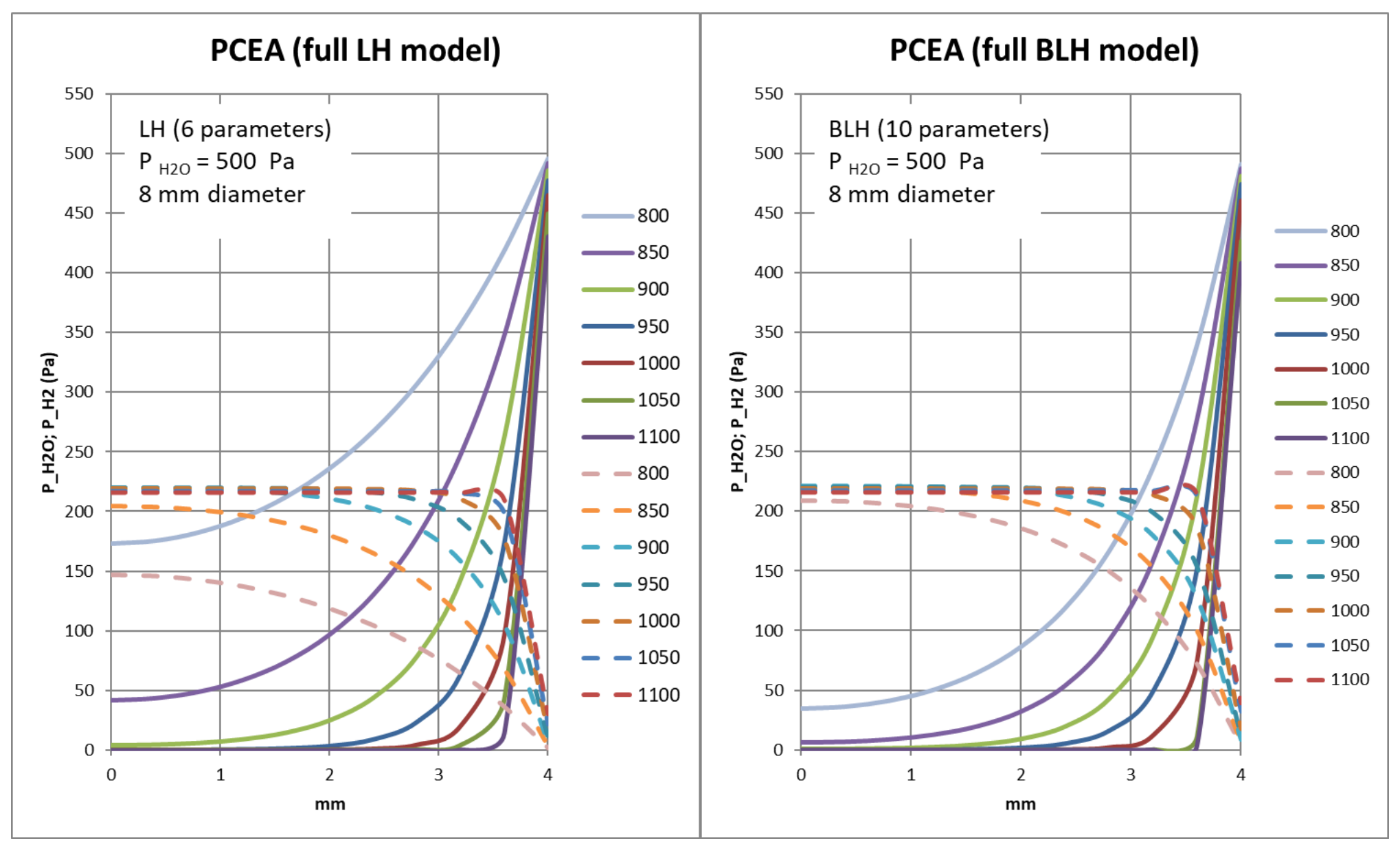



APPENDIX D. WATER PENETRATION AND HYDROGEN BUILDUP PROFILES IN GRAPHITE NBG-17
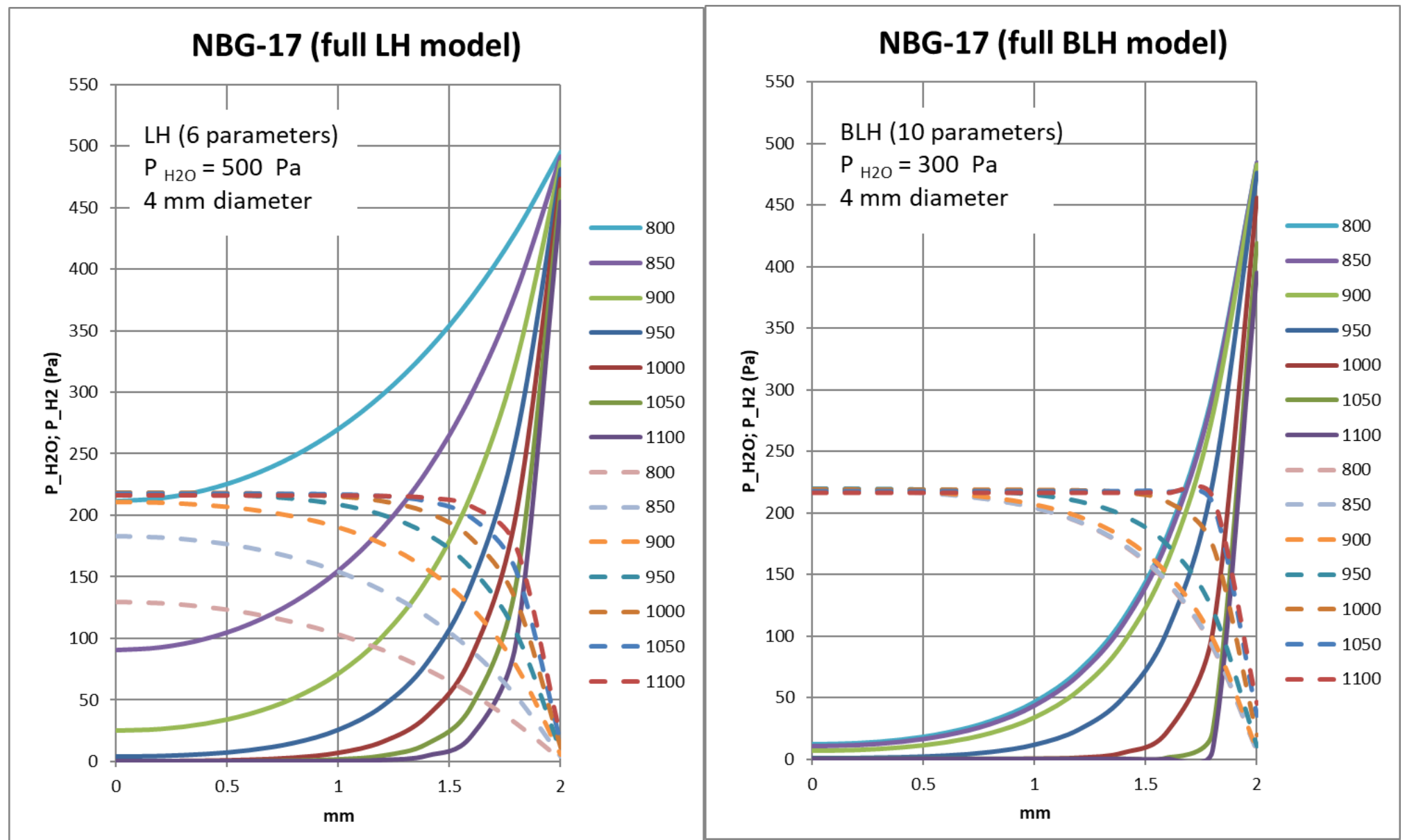

APPENDIX E. WATER PENETRATION AND HYDROGEN BUILDUP PROFILES IN GRAPHITE IG-110

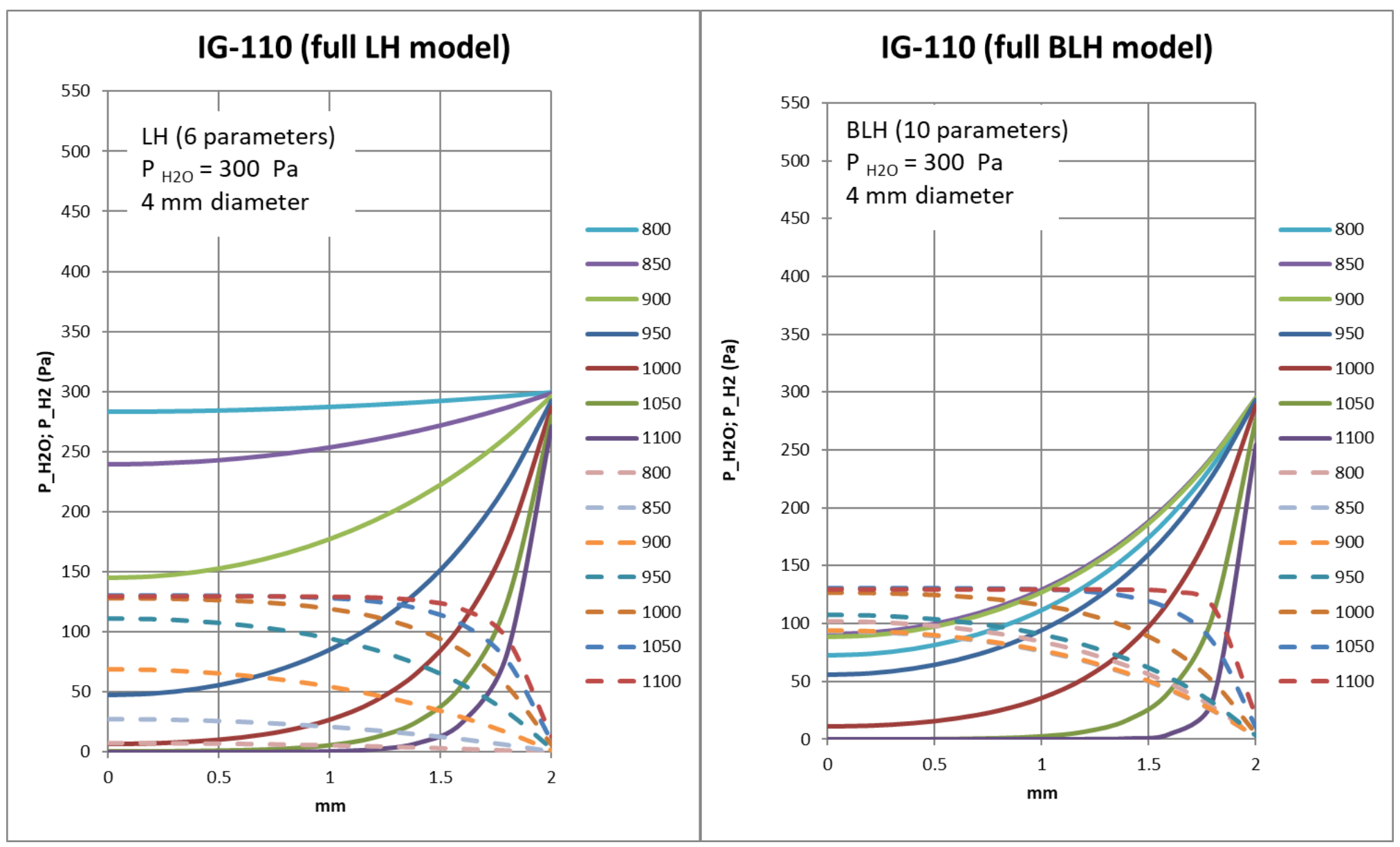


Models corrected by a temperature-dependent Knudsen diffusion coefficient calculated for water in $5 \mathrm{~nm}$ pores

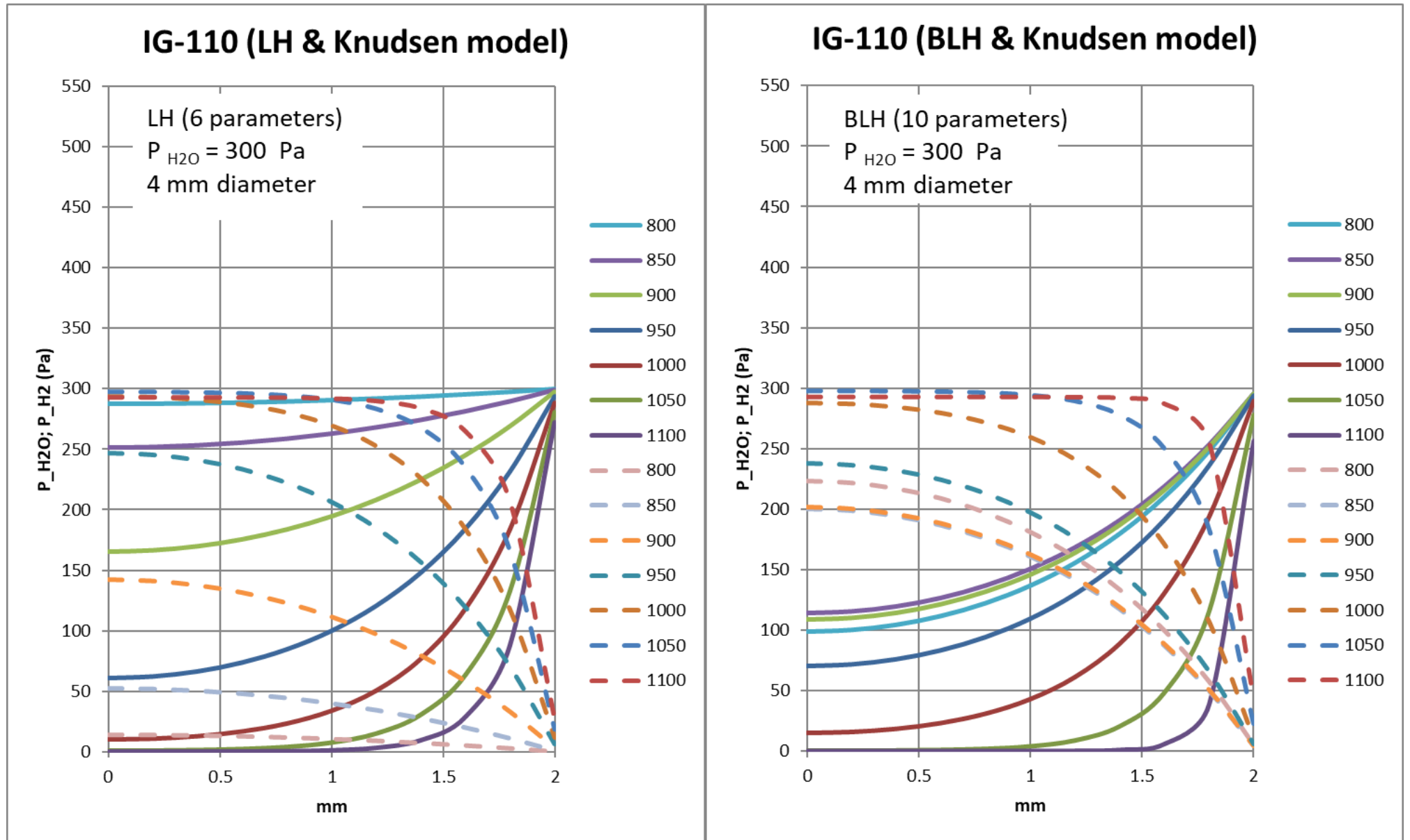


APPENDIX F. WATER PENETRATION AND HYDROGEN BUILDUP PROFILES IN GRAPHITE 2114
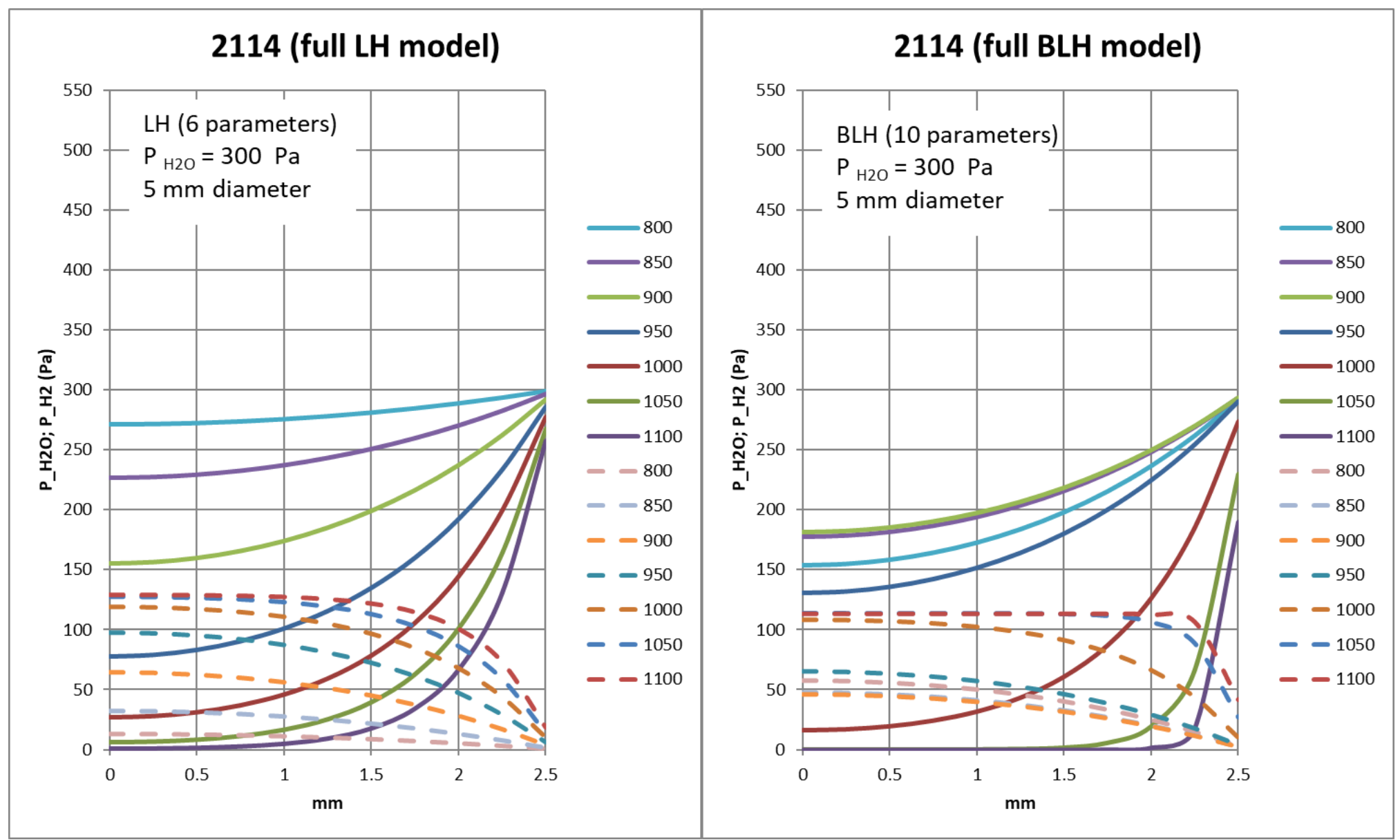
Models corrected by a temperature-dependent Knudsen diffusion coefficient calculated for water in $5 \mathrm{~nm}$ pores

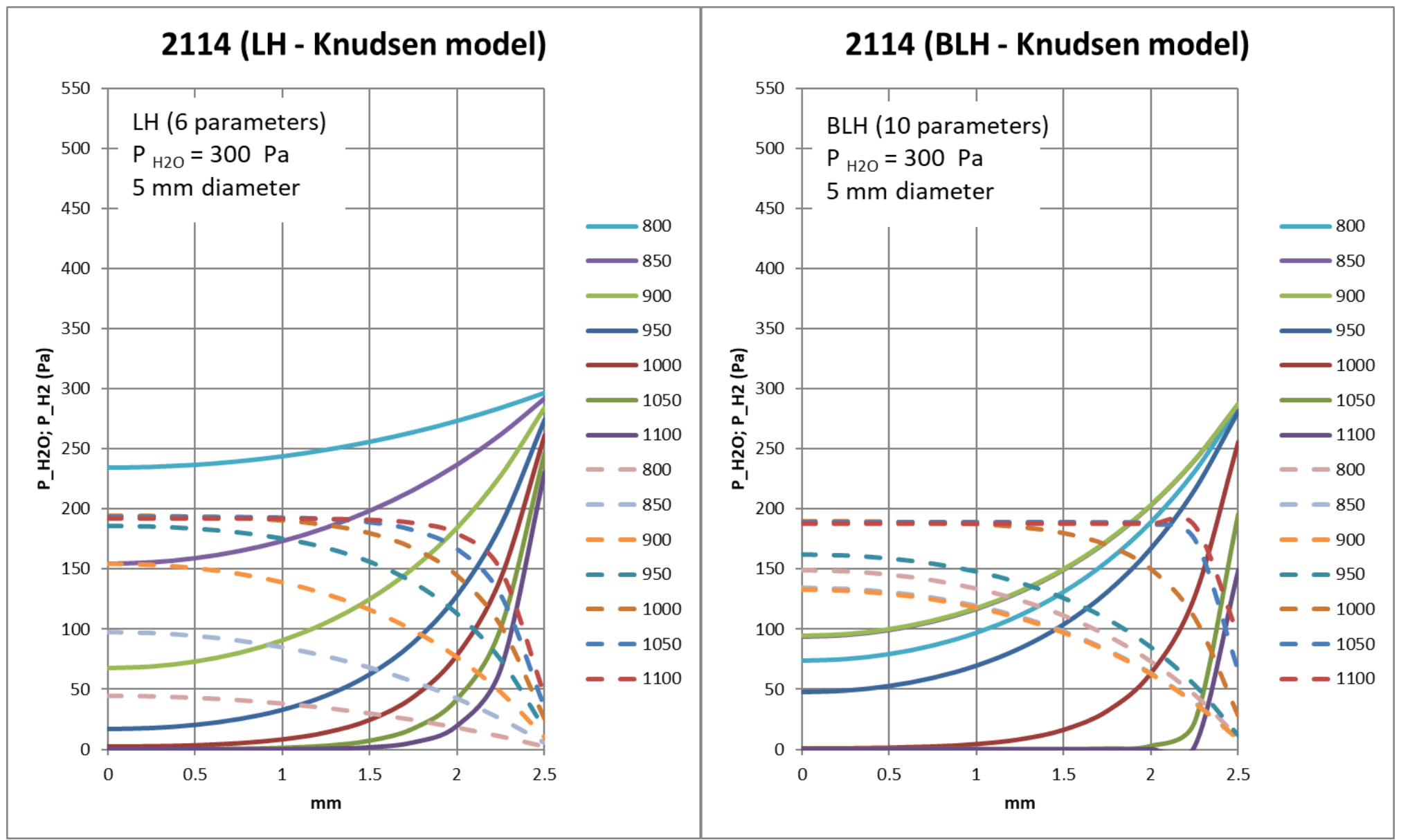

